\title{
Simulating cosmic ray physics on a moving mesh
}

\author{
C. Pfrommer ${ }^{1 \star}$, R. Pakmor ${ }^{1}$, K. Schaal ${ }^{1,2}$, C. M. Simpson ${ }^{1}$, and V. Springel ${ }^{1,2}$ \\ ${ }^{1}$ Heidelberger Institut für Theoretische Studien, Schloss-Wolfsbrunnenweg 35, 69118 Heidelberg, Germany \\ ${ }^{2}$ Zentrum für Astronomie der Universität Heidelberg, Astronomisches Recheninstitut, Mönchhofstr. 12-14, 69120 Heidelberg, Germany
}

\begin{abstract}
We discuss new methods to integrate the cosmic ray (CR) evolution equations coupled to magneto-hydrodynamics (MHD) on an unstructured moving mesh, as realised in the massively parallel AREPO code for cosmological simulations. We account for diffusive shock acceleration of CRs at resolved shocks and at supernova remnants in the interstellar medium (ISM), and follow the advective CR transport within the magnetised plasma, as well as anisotropic diffusive transport of CRs along the local magnetic field. CR losses are included in terms of Coulomb and hadronic interactions with the thermal plasma. We demonstrate the accuracy of our formalism for CR acceleration at shocks through simulations of plane-parallel shock tubes that are compared to newly derived exact solutions of the Riemann shock tube problem with CR acceleration. We find that the increased compressibility of the post-shock plasma due to the produced CRs decreases the shock speed. However, CR acceleration at spherically expanding blast waves does not significantly break the self-similarity of the Sedov-Taylor solution; the resulting modifications can be approximated by a suitably adjusted, but constant adiabatic index. In first applications of the new CR formalism to simulations of isolated galaxies and cosmic structure formation, we find that CRs add an important pressure component to the ISM that increases the vertical scale height of disk galaxies, and thus reduces the star formation rate. Strong external structure formation shocks inject CRs into the gas, but the relative pressure of this component decreases towards halo centres as adiabatic compression favours the thermal over the CR pressure.
\end{abstract}

Key words: cosmic rays, (magnetohydrodynamics) MHD, shock waves, galaxies: formation, cosmology: large-scale structure of Universe, methods: numerical

\section{INTRODUCTION}

Understanding the physics of galaxy formation is arguably one of the most complicated problems in modern astrophysics. A large body of theoretical work based on cosmological simulations and semi-analytic models has demonstrated that so-called feedback processes by stellar winds and radiation fields, supernovae, and active galactic nuclei (AGNs) appear to be critical in obtaining realistic galaxy populations (e.g. Schaye et al. 2010; Guedes et al. 2011; Puchwein \& Springel 2013; Hopkins et al. 2014; Marinacci et al. 2014; Henriques et al. 2015; Vogelsberger et al. 2014; Schaye et al. 2015). These processes are invoked to slow down star formation to the small observed rates, to move gas and metals out of galaxies into the intergalactic medium by means of galactic winds, to obtain a realistic mix of early- and latetype galaxies, to quench star formation in elliptical galaxies,

^ e-mail: christoph.pfrommer@h-its.org (CP) and to balance radiative cooling of low-entropy gas at the centres of galaxy clusters so that global cluster observables agree with observations in the $\mathrm{X}$-ray and micro-wave regime via the thermal Sunyaev-Zel'dovich effect (Kravtsov \& Borgani 2012; Battaglia et al. 2012a,b, 2013; McCarthy et al. 2014, 2016; Dolag et al. 2016).

While the recent progress of galaxy formation models is remarkable, it still comes with the caveat that the involved feedback has typically been modelled empirically and tuned to match observed galaxy scaling relations, weakening the predictive power of the corresponding calculations. In particular, feedback in hydrodynamical simulations of galaxy formation has thus far often been implemented very coarsely, for example based on explicit subgrid models that aim to represent the unresolved, multi-phase structure of the ISM with an effective description that still yields the correct average star formation rate (Springel \& Hernquist 2003; Schaye \& Dalla Vecchia 2008). The physics behind galactic winds and outflows remains especially unclear, and is sometimes 
treated in a purely phenomenological way where the wind velocity and momentum flux are prescribed (Oppenheimer \& Davé 2006). Similarly, in order to prevent too many stars from precipitating out of the hot phase of the intra-cluster medium (ICM), feedback from AGN has often been modelled by estimating accretion rates with a simple Bondi prescription and injecting thermal energy as feedback (Di Matteo et al. 2005; Springel et al. 2005).

However, rather than just depositing thermal energy by supernovae or AGNs, a physically more correct solution may involve the formation of a non-thermal relativistic particle population (i.e., in CRs), created through the process of diffusive shock acceleration at expanding supernova remnants (e.g., Jubelgas et al. 2008) or in relativistic jets powered by AGNs (e.g., Sijacki et al. 2008). Non-thermal energy is dissipated on a longer timescale than thermal energy because CR cooling is generally less efficient than the radiation cooling of a thermal plasma (Enßlin et al. 2007). As the non-thermal energy is not observable through X-ray emission or other thermal observables, the (temporary) storage of feedback energy in CRs also avoids problems with the overproduction of these observables. Moreover, the CR pressure force could accelerate the ambient ISM and drive powerful galactic outflows and winds.

Another major contender for the physical basis of feedback in galaxies lies in the momentum and energy deposition of ultra-violet radiation. Momentum-driven winds can form when radiation pressure acts efficiently on dust grains and atomic lines in dense gas and imparts momentum kicks that can expel the gas if it exceeds the escape velocity. While this mechanism has been argued to provide efficient feedback during the formation of galaxies, possibly even explaining strong outflows in starburst galaxies (Murray et al. 2005; Thompson et al. 2005), direct radiation-hydrodynamical simulations of simplified set-ups of the Rayleigh-Taylor instability (Krumholz \& Thompson 2012) or full galaxy-scale models (Rosdahl et al. 2015; Skinner \& Ostriker 2015) fail to see these strong winds. This suggests that radiation feedback is more gentle and less effective than assumed in some subgrid prescriptions. Also, the high dust opacities needed for radiation pressure to be efficient are unlikely to be realised in Milky Way-type galaxies, in particular at larger galactocentric radii.

On the other hand, CRs and magnetic fields are observed to be in pressure equilibrium with the turbulence in the midplane of the Milky Way (Boulares \& Cox 1990). This could be naturally explained if it is the outcome of a self-regulated feedback loop where CRs and magnetic fields provide the main wind driving mechanism, as suggested by a number of theoretical works (Ipavich 1975; Breitschwerdt et al. 1991; Zirakashvili et al. 1996; Ptuskin et al. 1997; Breitschwerdt et al. 2002; Socrates et al. 2008; Everett et al. 2008, 2010; Samui et al. 2010; Dorfi \& Breitschwerdt 2012) and local three-dimensional (3D) simulations of the ISM (Hanasz et al. 2013; Girichidis et al. 2016).

In comparison to other wind-driving mechanisms, CRs have a number of properties that make them advantageous for driving winds: (i) their pressure drops less quickly upon adiabatic expansion than the thermal pressure due to their softer equation of state $\left(P_{\mathrm{cr}} \propto \rho^{\gamma_{\mathrm{cr}}}\right.$ with $\left.\gamma_{\mathrm{cr}}=4 / 3\right)$, (ii) CRs cool less efficiently than the thermal gas and can hold on to their energy for longer time scales, and (iii) the CRs can energise the wind as it rises from the disk with a rate that depends on the ratio of the advection-to-streaming speed, thus maintaining the outflows in a warm/hot state and acting like a 'CR battery'. Polarised radio observations of radio haloes in edge-on galaxies reveal poloidal field lines at the interface of the ISM and the galactic halo (e.g., Tüllmann et al. 2000), which coincides with the wind launching site: this argues for a dynamical mechanism that is responsible for reorienting the preferentially toroidal field in galactic disks by means of anisotropic CR transport along the magnetic field.

If the CR pressure in a disk is super-critical, i.e. if it is unstable to a buoyancy instability, CR-loaded gas will start to rise from the disk and drive a Parker instability (Rodrigues et al. 2016), resulting in a poloidal ('open') field configuration. Pioneering numerical work has demonstrated that CR-driven winds can expel ISM from the disk and efficiently regulate star formation in $3 \mathrm{D}$ hydrodynamic simulations of galaxy formation (Uhlig et al. 2012; Booth et al. 2013; Salem \& Bryan 2014; Salem et al. 2014; Ruszkowski et al. 2016). Unlike the classical energy- or momentumdriven wind solutions, CR-driven winds impart not only energy and momentum to the ISM at the wind base, but can also continuously repower the plasma during its ascent because of dynamic and thermal coupling of CRs to the plasma.

The ability of CRs to drive winds is intimately tied to their transport processes. In particular, the fast streaming of CRs along the magnetic field excites Alfvén waves through the streaming instability (Lerche 1967; Kulsrud \& Pearce 1969). Scattering off of this wave field limits the CRs' bulk speed in turn. These waves are then damped, effectively transferring $\mathrm{CR}$ energy and momentum to the thermal plasma. Hence CRs exert a pressure on the thermal plasma by means of scattering off of Alfvén waves. Interestingly, winds driven by $\mathrm{CR}$ streaming are characterised by an increasing mass loading factor $\eta_{\mathrm{m}}=\dot{M} / \dot{M}_{*}$ of the galactic wind towards smaller galaxies (where $\dot{M}$ and $\dot{M}_{*}$ denote the wind mass loss and star formation rate, respectively, see Uhlig et al. 2012). This mass scaling of $\eta_{\mathrm{m}}$ may be required to yield a strongly decreasing star conversion efficiency of baryons towards dwarf galaxies and thus makes CR physics a prime candidate for the physical mechanism underlying this conundrum of galaxy formation.

In addition, CRs may hold the key to understanding a similar problem in giant elliptical galaxies located at the centres of galaxy groups and clusters. In the absence of heating processes, the hot gaseous atmospheres of these objects are expected to cool and feed star formation at rates up to several hundred $\mathrm{M}_{\odot} \mathrm{yr}^{-1}$ (see Peterson \& Fabian 2006, for a review). Instead, the observed gas cooling and star formation rates are reduced to levels substantially below those expected from unimpeded cooling flows. High-resolution Chandra and XMM-Newton observations of clusters have provided evidence that radiative cooling is offset by an unidentified heating process, which is associated with the AGN jetinflated radio lobes that are co-local with the cavities seen in the X-ray maps. The interaction of cooling gas, subsequent star formation, and nuclear activity seems to be tightly coupled to a self-regulated feedback loop (for reviews, see McNamara \& Nulsen 2007, 2012). While the energetics associated with AGN feedback are sufficient to balance radiative cool- 
ing, how the available energy is coupled to the cluster gas is less clear.

Several physical processes have been proposed to mediate the heating, including dissipation of turbulent energy excited by the rising AGN lobes (Zhuravleva et al. 2014). Alternatively, a net outward flux of streaming CRs may provide a means to stably heat the cooling cluster plasma by the excitation of resonant Alfvén waves. Once generated, they experience non-linear Landau damping or decay via a cascading process as a result of strong external turbulence, and eventually dissipate locally (Loewenstein et al. 1991; Guo \& Oh 2008; Enßlin et al. 2011; Fujita \& Ohira 2012; Wiener et al. 2013; Pfrommer 2013; Jacob \& Pfrommer 2016a,b).

This picture is supported by the widespread evidence for non-thermal activity in galaxy clusters, which also manifests itself on cluster scales through extended synchrotron emission in the form of radio haloes and peripheral relics (for a review, see Brunetti \& Jones 2014). Despite a large body of work, the origin of radio haloes and the nature of seed electrons that are energised in radio relics are still open problems. This is because in clusters different plausible acceleration mechanisms are operating that range from diffusive shock acceleration of relativistic electrons to injection as secondaries in hadronic interactions of relativistic protons with the dense cluster gas, and even turbulent re-acceleration through interactions with the compressible cluster turbulence. One or a combination of these processes could be responsible for those enigmatic radio phenomena. Cosmological MHD simulations with CR physics are indispensable for solving these puzzles by evolving the Fokker-Planck equation for the CR distribution through cosmic time, following in detail the $\mathrm{CR}$ acceleration and transport into galaxy clusters (Miniati et al. 2001a,b; Pfrommer et al. 2007, 2008; Pfrommer 2008; Pinzke \& Pfrommer 2010; Vazza et al. 2012; Donnert et al. 2013; Pinzke et al. 2013, 2015).

The above considerations provide ample motivation for outfitting modern hydrodynamical cosmological simulation codes with a numerically efficient and accurate treatment of CR physics coupled to MHD. This is the goal of this paper, which aims to provide a comprehensive exposition of the most relevant CR physics, and a description of our numerical implementation of it in the AREPO moving-mesh code. We discuss a set of tests of the new code, focusing in particular on the modifications of strong shocks due to the inlined CR acceleration. We also present a set of first applications in the form of isolated disk galaxies and basic cosmological simulations of structure formation. In a companion paper (Pakmor et al. 2016b), we provide further details on the technical implementations of our anisotropic diffusion solver, and in two further companion studies, we apply our new methodology to wind formation in disk galaxies (Pakmor et al. 2016c) and to the regulation of the ISM by individual supernova explosions (Simpson et al. 2016).

This paper is organised as follows. In Section 2, we present the basic system of MHD equations with CRs in physical coordinates, on a cosmologically expanding background, and describe our implementation. In Section 3, we detail our modelling of non-adiabatic processes of CRs that include diffusive shock acceleration, injection at supernova remnants, and collisional loss processes such as Coulomb and hadronic interactions with the ambient plasma. In Section 4 , we present our results on plane parallel shock tubes and a spherically expanding Sedov-Taylor blast wave with CR shock acceleration. We also discuss MHD simulations of galaxy formation that include advective CR transport, and initial results on cosmological simulations that account for $\mathrm{CR}$ acceleration at structure formation shocks. In Section 5, we summarise our main findings and conclusions. Finally, in Appendix A, we review the basic considerations underlying the derivation of CR hydrodynamics, and in Appendices B and $\mathrm{C}$ we detail our novel derivation of the exact solutions of the Riemann shock-tube problem when CR acceleration with and without a pre-existing population of CRs is included.

\section{BASIC EQUATIONS}

\subsection{Magneto-hydrodynamics with cosmic rays}

The equations of ideal magneto-hydrodynamics of a twofluid medium composed of thermal gas and CRs can be written as a system of conservation laws,

$$
\frac{\partial \boldsymbol{U}}{\partial t}+\boldsymbol{\nabla} \cdot \mathbf{F}=\boldsymbol{S}
$$

for a vector of conserved variables $\boldsymbol{U}$, a flux function $\mathbf{F} \equiv$ $\mathbf{F}(\boldsymbol{U})$, and source terms $\boldsymbol{S}$. Those are given in the local restframe by (see Appendix A)

$$
\begin{aligned}
& \boldsymbol{U}=\left(\begin{array}{c}
\rho \\
\rho \boldsymbol{v} \\
\varepsilon \\
\varepsilon_{\mathrm{cr}} \\
\boldsymbol{B}
\end{array}\right), \quad \mathbf{F}=\left(\begin{array}{c}
\rho \boldsymbol{v} \\
\rho \boldsymbol{v} \boldsymbol{v}^{\mathrm{T}}+P \mathbf{1}-\boldsymbol{B} \boldsymbol{B}^{\mathrm{T}} \\
(\varepsilon+P) \boldsymbol{v}-\boldsymbol{B}(\boldsymbol{v} \cdot \boldsymbol{B}) \\
\varepsilon_{\mathrm{cr}} \boldsymbol{v}+\left(\varepsilon_{\mathrm{cr}}+P_{\mathrm{cr}}\right) \boldsymbol{v}_{\mathrm{st}}-\kappa_{\varepsilon} \boldsymbol{b}\left(\boldsymbol{b} \cdot \boldsymbol{\nabla} \varepsilon_{\mathrm{cr}}\right) \\
\boldsymbol{B v}^{\mathrm{T}}-\boldsymbol{v} \boldsymbol{B}^{\mathrm{T}}
\end{array}\right), \\
& \boldsymbol{S}=\left(\begin{array}{c}
0 \\
\mathbf{0} \\
P_{\mathrm{cr}} \boldsymbol{\nabla} \cdot \boldsymbol{v}-\boldsymbol{v}_{\mathrm{st}} \cdot \boldsymbol{\nabla} P_{\mathrm{cr}}+\Lambda_{\mathrm{th}}+\Gamma_{\mathrm{th}} \\
-P_{\mathrm{cr}} \boldsymbol{\nabla} \cdot \boldsymbol{v}+\boldsymbol{v}_{\mathrm{st}} \cdot \boldsymbol{\nabla} P_{\mathrm{cr}}+\Lambda_{\mathrm{cr}}+\Gamma_{\mathrm{cr}} \\
\mathbf{0}
\end{array}\right)
\end{aligned}
$$

where we used the Heaviside-Lorentz system of units. $P$ is the total pressure due to thermal gas, CRs, and magnetic fields and $\varepsilon$ is the total energy density excluding CRs,

$$
\begin{aligned}
P & =P_{\mathrm{th}}+P_{\mathrm{cr}}+\frac{B^{2}}{2}, \\
\varepsilon & =\varepsilon_{\mathrm{th}}+\frac{\rho \boldsymbol{v}^{2}}{2}+\frac{\boldsymbol{B}^{2}}{2} .
\end{aligned}
$$

The local gas density, velocity, and magnetic field strength are given by $\rho, \boldsymbol{v}$ and $\boldsymbol{B}$, respectively. The thermal and CR energy densities are given by $\varepsilon_{\mathrm{th}}$ and $\varepsilon_{\mathrm{cr}}$, respectively. The unit vector along the local magnetic field is denoted by $\boldsymbol{b}=$ $\boldsymbol{B} /|\boldsymbol{B}|$ and $\mathbf{1}$ is the unit rank-two tensor. The kinetic energyweighted spatial diffusion coefficient of CRs is denoted by $\kappa_{\varepsilon}$ (see also Appendix A) and the CR streaming velocity is given by

$$
\boldsymbol{v}_{\mathrm{st}}=-\boldsymbol{v}_{\mathrm{A}} \operatorname{sgn}\left(\boldsymbol{B} \cdot \boldsymbol{\nabla} P_{\mathrm{cr}}\right)=-\frac{\boldsymbol{B}}{\sqrt{\rho}} \frac{\boldsymbol{B} \cdot \boldsymbol{\nabla} P_{\mathrm{cr}}}{\left|\boldsymbol{B} \cdot \boldsymbol{\nabla} P_{\mathrm{cr}}\right|},
$$


implying that the CR streaming velocity is oriented along magnetic fields lines down the CR pressure gradient with a velocity that corresponds in magnitude to the velocity of Alfvén waves, $\boldsymbol{v}_{\mathrm{A}}$. Hence, the $\mathrm{CR}$ source term due to the generation of resonant Alfvén waves in the CR equation is always a loss term,

$$
\boldsymbol{v}_{\mathrm{st}} \cdot \boldsymbol{\nabla} P_{\mathrm{cr}}=-\frac{1}{\sqrt{\rho}} \frac{\left(\boldsymbol{B} \cdot \boldsymbol{\nabla} P_{\mathrm{cr}}\right)^{2}}{\left|\boldsymbol{B} \cdot \boldsymbol{\nabla} P_{\mathrm{cr}}\right|}<0 .
$$

Equivalently, the corresponding term in the thermal energy equation is always a gain term $\left(-\boldsymbol{v}_{\mathrm{st}} \cdot \boldsymbol{\nabla} P_{\mathrm{cr}}>0\right) .{ }^{1}$ Furthermore, there are explicit gain and loss terms $\left(\Gamma_{i}\right.$ and $\Lambda_{i}$ with $i \in\{$ th, $\mathrm{cr}\})$ for the thermal and CR energy density, respectively. If there are no explicit gain and loss terms, we clearly see that the total energy (volume integral of $\partial\left(\varepsilon+\varepsilon_{\mathrm{cr}}\right) / \partial t$ ) is conserved, i.e., that the source terms of these two equations vanish identically. To close the system of equations, we assume an equation of state for the thermal gas as well as for the CRs

$$
\begin{aligned}
& P_{\mathrm{th}}=\left(\gamma_{\mathrm{th}}-1\right) \varepsilon_{\mathrm{th}}, \\
& P_{\mathrm{cr}}=\left(\gamma_{\mathrm{cr}}-1\right) \varepsilon_{\mathrm{cr}},
\end{aligned}
$$

with $\gamma_{\text {th }}=5 / 3$ and $\gamma_{\mathrm{cr}}=4 / 3$ in the relativistic limit. Additionally, the magnetic field has to fulfil the divergence constraint $\boldsymbol{\nabla} \cdot \boldsymbol{B}=0$. To understand the physics of CR transport, we can rewrite the equation for the CR energy density and obtain

$$
\begin{aligned}
& \frac{\partial \varepsilon_{\mathrm{cr}}}{\partial t}+\boldsymbol{\nabla} \cdot {\left[\varepsilon_{\mathrm{cr}}\left(\boldsymbol{v}+\boldsymbol{v}_{\mathrm{st}}\right)-\kappa_{\varepsilon} \boldsymbol{b}\left(\boldsymbol{b} \cdot \boldsymbol{\nabla} \varepsilon_{\mathrm{cr}}\right)\right] } \\
&=-P_{\mathrm{cr}} \boldsymbol{\nabla} \cdot\left(\boldsymbol{v}+\boldsymbol{v}_{\mathrm{st}}\right)+\Lambda_{\mathrm{cr}}+\Gamma_{\mathrm{cr}} .
\end{aligned}
$$

This demonstrates that the spatial transport of CR energy density is a superposition of advection with the frame propagating at velocity $\boldsymbol{v}+\boldsymbol{v}_{\text {st }}$, as well as anisotropic diffusion along magnetic field lines with respect to that frame. In addition to the explicit source terms $\left(\Gamma_{\mathrm{cr}}\right.$ and $\left.\Lambda_{\mathrm{cr}}\right)$, CRs experience adiabatic gains and losses depending on whether the Alfvén frame is compressed $\left(\boldsymbol{\nabla} \cdot\left(\boldsymbol{v}+\boldsymbol{v}_{\mathrm{st}}\right)<0\right)$ or expanded in the laboratory frame.

The physical picture underlying this transport equation can be understood with the following considerations. CRs with energies around the proton rest mass energy dominate the CR pressure (Enßlin et al. 2007). CRs that propagate faster than the Alfvén velocity excite Alfvén waves through the CR streaming instability (Kulsrud \& Pearce 1969). Scattering off of this self-excited wave field isotropises the CRs' pitch angles, thereby confining the pressure-carrying CRs almost perfectly to the Alfvén wave frame (Wiener et al. 2013, 2016). ${ }^{2}$ As a result, those CRs stream along magnetic fields with the streaming velocity $\boldsymbol{v}_{\text {st }}$ (equation 5 ).

On the other hand, effective damping processes (such as

\footnotetext{
1 Note that we refrain from explicitly integrating the equation for the Alfvén wave energy that is resonantly generated by the streaming CRs since this energy is quickly dissipated.

${ }^{2}$ Note that more relativistic CRs with Lorentz factors $\gamma \gtrsim 100$ can drift super-Alfvénically due to the weaker coupling of these CRs to the Alfvén wave frame. This is because the streaming instability growth rate scales with the number of CRs, which is steeply declining with energy for usual CR power-law distributions (Wiener et al. 2013; Recchia et al. 2016).
}

ion-neutral interactions) can lead to an incomplete CR confinement. Externally sourced turbulence can interact with CRs, leading to more spatial confinement of CRs. Effectively, these effects can be described by a diffusion process with an energy-weighted spatial diffusion coefficient $\kappa_{\varepsilon}$. In the absence of CR diffusion $\left(\kappa_{\varepsilon}=0\right)$ and explicit gains and losses $\left(\Gamma_{i}=0=\Lambda_{i}\right.$ for $\left.i \in\{\mathrm{th}, \mathrm{cr}\}\right)$, the equation for the CR energy density (9) states that CRs only experience thermodynamically reversible changes of their population as a result of adiabatic expansion/compression of the gas as well as adiabatic losses due to CR streaming. These streaming losses irreversibly heat the thermal gas by non-linear Landau damping or scattering off of strong external turbulence that cascade the energy down to the dissipation scale. However, the supply of mechanical energy, e.g., in form of turbulence can adiabatically add CR energy through volume-compression work done on the CRs.

CR energy sources $\left(\Gamma_{\mathrm{cr}}\right)$ are provided by diffusive shock acceleration at supernova remnants, at cosmological formation shocks, and in relativistic jets from active galactic nuclei. Energy gain through second-order Fermi acceleration by means of momentum space diffusion is another source of CR energy. CR energy sinks $\left(\Lambda_{\mathrm{cr}}\right)$ are provided by hadronic interactions of CRs with thermal gas protons, Coulomb and ionisation interactions, which will be detailed below.

\subsection{MHD with cosmic rays in comoving coordinates}

In the previous section, all spatial derivatives are taken with respect to physical coordinates $r$. In an expanding universe, it is convenient to introduce spatial coordinates $\boldsymbol{x}$ comoving with the cosmological expansion so that only gas motions with respect to that comoving rest frame need to be computed. We parameterise the global expansion of the universe with the time-dependent scale factor $a(t)$ that obeys Friedmann's equations. To this end, we define a set of 'comoving' variables (denoted with a subscript 'c') for velocity, mass density, magnetic field, thermal and CR pressure:

$$
\begin{aligned}
r & =a \boldsymbol{x}, & \boldsymbol{u} & =\boldsymbol{v}-\dot{\boldsymbol{a}} \boldsymbol{x}, \\
\rho & =\rho_{\mathrm{c}} a^{-3}, & \boldsymbol{B} & =\boldsymbol{B}_{\mathrm{c}} a^{-2}, \\
P_{\mathrm{th}} & =P_{\mathrm{th}, \mathrm{c}} a^{-3}, & P_{\mathrm{cr}} & =P_{\mathrm{cr}, \mathrm{c}} a^{-4}, \\
\varepsilon_{\mathrm{th}} & =\varepsilon_{\mathrm{th}, \mathrm{c}} a^{-3}, & \varepsilon_{\mathrm{cr}} & =\varepsilon_{\mathrm{cr}, \mathrm{c}} a^{-4} .
\end{aligned}
$$

Here, $\boldsymbol{v}=\dot{\boldsymbol{r}}$ is the physical velocity and $\boldsymbol{u}=a \dot{\boldsymbol{x}}$ is the peculiar velocity. With the exception of the magnetic field and CR pressure, these transformations are standard definitions. The adopted definitions for $\boldsymbol{B}_{\mathrm{c}}$ and $P_{\mathrm{cr}, \mathrm{c}}$ ensure that there are no cosmological source terms in the induction equation and the equation for the CR energy density. The latter property is a consequence of the assumption of a constant $\gamma_{\mathrm{cr}}$. More realistic models of CRs that go beyond this simplification would have to account for a cosmological source term. The transformation to comoving coordinates implies the following transformation of derivatives,

$$
\boldsymbol{\nabla} \equiv \boldsymbol{\nabla}_{r} \rightarrow \frac{1}{a} \boldsymbol{\nabla}_{x} \quad \text { and } \quad \frac{\partial}{\partial t} \rightarrow \frac{\partial}{\partial t}-H \boldsymbol{x} \cdot \boldsymbol{\nabla}_{x},
$$

where $H \equiv H(a)=\dot{a} / a$ is the Hubble function, the transformed time derivatives are defined at constant comoving 
position $\boldsymbol{x}$, and $\boldsymbol{\nabla}_{x}$ is the vector of spatial derivatives with respect to $\boldsymbol{x}$. Adopting our replacement rules, we find for the comoving evolution equations

$$
\frac{\partial \boldsymbol{U}_{\mathrm{c}}}{\partial t}+\frac{1}{a} \boldsymbol{\nabla}_{x} \cdot \mathbf{F}_{\mathrm{c}}=\boldsymbol{S}_{\mathrm{c}},
$$

for a vector of conserved comoving variables $\boldsymbol{U}_{\mathrm{c}}$, a comoving flux function $\boldsymbol{F}_{\mathrm{c}} \equiv \mathbf{F}_{\mathrm{c}}\left(\boldsymbol{U}_{\mathrm{c}}\right)$, and comoving source terms $\boldsymbol{S}_{\mathrm{c}}$. Those are given in the local restframe by

$$
\begin{gathered}
\boldsymbol{U}_{\mathrm{c}}=\left(\begin{array}{c}
\rho_{\mathrm{c}} \\
\rho_{\mathrm{c}} \boldsymbol{u} \\
\varepsilon_{\mathrm{c}} \\
\varepsilon_{\mathrm{cr}, \mathrm{c}} \\
\boldsymbol{B}_{\mathrm{c}}
\end{array}\right), \mathbf{F}_{\mathrm{c}}\left(\begin{array}{c}
\rho_{\mathrm{c}} \boldsymbol{u} \\
\rho_{\mathrm{c}} \boldsymbol{u} \boldsymbol{u}^{\mathrm{T}}+P_{\mathrm{c}} \mathbf{1}-a^{-1} \boldsymbol{B}_{\mathrm{c}} \boldsymbol{B}_{\mathrm{c}}^{\mathrm{T}} \\
\left(\varepsilon_{\mathrm{c}}+P_{\mathrm{c}}\right) \boldsymbol{u}-\frac{\boldsymbol{B}_{\mathrm{c}}}{a}\left(\boldsymbol{u} \cdot \boldsymbol{B}_{\mathrm{c}}\right)+\frac{\boldsymbol{v}_{\mathrm{s}, \mathrm{c}}}{a^{3 / 2}} P_{\mathrm{cr}, \mathrm{c}} \\
\varepsilon_{\mathrm{cr}, \mathrm{c}} \boldsymbol{u}_{\mathrm{A}}-a^{-1} \kappa_{\varepsilon} \boldsymbol{b}\left(\boldsymbol{b} \cdot \boldsymbol{\nabla}_{x} \varepsilon_{\mathrm{cr}, \mathrm{c}}\right) \\
\boldsymbol{B}_{\mathrm{c}} \boldsymbol{u}^{\mathrm{T}}-\boldsymbol{u} \boldsymbol{B}_{\mathrm{c}}^{\mathrm{T}} \\
0 \\
-H \rho_{\mathrm{c}} \boldsymbol{u} \\
\boldsymbol{S}_{\mathrm{c}}=\left(\begin{array}{c}
\frac{P_{\mathrm{cr}, \mathrm{c}}}{a^{2}} \nabla_{x} \cdot \boldsymbol{u}_{\mathrm{A}}+\Gamma_{\mathrm{th}, \mathrm{c}}+\Lambda_{\mathrm{th}, \mathrm{c}}-H\left(2 \varepsilon_{\mathrm{c}}-\frac{\boldsymbol{B}_{\mathrm{c}}^{2}}{2 a}\right) \\
-\frac{P_{\mathrm{cr}, \mathrm{c}}}{a} \nabla_{x} \cdot \boldsymbol{u}_{\mathrm{A}}+\Gamma_{\mathrm{cr}, \mathrm{c}}+\Lambda_{\mathrm{cr}, \mathrm{c}}+H \varepsilon_{\mathrm{cr}, \mathrm{c}}\left(4-3 \gamma_{\mathrm{cr}}\right) \\
\mathbf{0}
\end{array}\right) .
\end{array}\right.
\end{gathered}
$$

Here, we introduced the total comoving pressure $\left(P_{\mathrm{c}}\right)$ and the total comoving energy density $\left(\varepsilon_{\mathrm{c}}\right)$ (excluding the CR energy density),

$$
\begin{aligned}
P_{\mathrm{c}} & =P_{\mathrm{th}, \mathrm{c}}+\frac{1}{a}\left(P_{\mathrm{cr}, \mathrm{c}}+\frac{\boldsymbol{B}_{\mathrm{c}}^{2}}{2}\right)=P_{\mathrm{th}, \mathrm{c}}+\tilde{P}_{\mathrm{cr}, \mathrm{c}}+\frac{\tilde{B}_{\mathrm{c}}^{2}}{2}, \\
\varepsilon_{\mathrm{c}} & =\varepsilon_{\mathrm{th}, \mathrm{c}}+\frac{\rho_{\mathrm{c}}}{2} \boldsymbol{u}^{2}+\frac{\boldsymbol{B}_{\mathrm{c}}^{2}}{2 a} .
\end{aligned}
$$

In practice, the AREPO code employs a redefinition of the CR pressure, $\tilde{P}_{\mathrm{cr}, \mathrm{c}}=P_{\mathrm{cr}, \mathrm{c}} / a$, and magnetic field, $\tilde{\boldsymbol{B}}_{\mathrm{c}}=\boldsymbol{B}_{\mathrm{c}} / \sqrt{a}$, to facilitate the notation of the total pressure. We further define the comoving gain and loss terms for the thermal and CR energy densities, respectively,

$$
\begin{array}{ll}
\Gamma_{\mathrm{th}}=a^{-3} \Gamma_{\mathrm{th}, \mathrm{c}}, & \Lambda_{\mathrm{th}}=a^{-3} \Lambda_{\mathrm{th}, \mathrm{c}}, \\
\Gamma_{\mathrm{cr}}=a^{-4} \Gamma_{\mathrm{cr}, \mathrm{c}}, & \Lambda_{\mathrm{cr}}=a^{-4} \Lambda_{\mathrm{cr}, \mathrm{c}} .
\end{array}
$$

The peculiar velocity of the frame comoving with forward Afvén waves that are excited by streaming CRs is given by

$$
\boldsymbol{u}_{\mathrm{A}}=\boldsymbol{u}+a^{-1 / 2} \boldsymbol{v}_{\mathrm{st}, \mathrm{c}}, \quad \text { with } \quad \boldsymbol{v}_{\mathrm{st}, \mathrm{c}}=-\operatorname{sgn}\left(\boldsymbol{B}_{\mathrm{c}} \cdot \boldsymbol{\nabla} P_{\mathrm{cr}, \mathrm{c}}\right) \frac{\boldsymbol{B}_{\mathrm{c}}}{\sqrt{\rho_{\mathrm{c}}}} .
$$

The cosmological source terms in the energy and momentum equations can be absorbed through a redefinition of velocity and total energy density via

$$
\begin{aligned}
\boldsymbol{w} & =a \boldsymbol{u}, \\
\tilde{\varepsilon}_{\mathrm{c}} & =a^{2} \varepsilon_{\mathrm{c}}=a^{5}\left(\varepsilon_{\mathrm{th}}+\frac{\rho}{2} \boldsymbol{u}^{2}+\boldsymbol{B}^{2}\right) .
\end{aligned}
$$

Substituting these variables into the cosmological MHD equations with CRs enables us to derive a set of equations in which the new variable $\boldsymbol{w}$ is used for the conservative variables while the fluxes are formulated with $\boldsymbol{u}$,

$$
\frac{\partial \tilde{\boldsymbol{U}}_{\mathrm{c}}}{\partial t}+\frac{1}{a} \boldsymbol{\nabla}_{x} \cdot \tilde{\mathbf{F}}_{\mathrm{c}}=\tilde{\boldsymbol{S}}_{\mathrm{c}}
$$

where we redefined the conservative variables $\tilde{\boldsymbol{U}}_{\mathrm{c}}$, fluxes $\tilde{\mathbf{F}}_{\mathrm{c}} \equiv$ $\tilde{\boldsymbol{F}}_{\mathrm{c}}\left(\tilde{\boldsymbol{U}}_{\mathrm{c}}\right)$, and source terms $\tilde{\boldsymbol{S}}_{\mathrm{c}}$ via

$$
\begin{aligned}
& \tilde{\boldsymbol{U}}_{\mathrm{c}}=\left(\begin{array}{c}
\rho_{\mathrm{c}} \\
\rho_{\mathrm{c}} \boldsymbol{w} \\
\tilde{\varepsilon}_{\mathrm{c}} \\
\varepsilon_{\mathrm{cr}, \mathrm{c}} \\
\boldsymbol{B}_{\mathrm{c}}
\end{array}\right), \tilde{\mathbf{F}}_{\mathrm{c}}=\left(\begin{array}{c}
\rho_{\mathrm{c}} \boldsymbol{u} \\
a\left(\rho_{\mathrm{c}} \boldsymbol{u} \boldsymbol{u}^{\mathrm{T}}+P_{\mathrm{c}} \mathbf{1}-a^{-1} \boldsymbol{B}_{\mathrm{c}} \boldsymbol{B}_{\mathrm{c}}^{\mathrm{T}}\right) \\
a^{2}\left[\left(\varepsilon_{\mathrm{c}}+P_{\mathrm{c}}\right) \boldsymbol{u}-\frac{\boldsymbol{B}_{\mathrm{c}}}{a}\left(\boldsymbol{u} \cdot \boldsymbol{B}_{\mathrm{c}}\right)+\frac{\boldsymbol{v}_{\mathrm{st}, \mathrm{c}}}{a^{3 / 2}} P_{\mathrm{cr}, \mathrm{c}}\right] \\
\varepsilon_{\mathrm{cr}, \mathrm{c}} \boldsymbol{u}_{\mathrm{A}}-a^{-1} \kappa_{\varepsilon} \boldsymbol{b}\left(\boldsymbol{b} \cdot \boldsymbol{\nabla}_{x} \varepsilon_{\mathrm{cr}, \mathrm{c}}\right) \\
\boldsymbol{B}_{\mathrm{c}} \boldsymbol{u}^{\mathrm{T}}-\boldsymbol{u} \boldsymbol{B}_{\mathrm{c}}^{\mathrm{T}}
\end{array}\right), \\
& \tilde{\boldsymbol{S}}_{\mathrm{c}}=\left(\begin{array}{c}
0 \\
\mathbf{0} \\
P_{\mathrm{cr}, \mathrm{c}} \boldsymbol{\nabla}_{x} \cdot \boldsymbol{u}_{\mathrm{A}}+a^{2}\left(\Gamma_{\mathrm{th}, \mathrm{c}}+\Lambda_{\mathrm{th}, \mathrm{c}}\right)+\frac{a H}{2} \boldsymbol{B}_{\mathrm{c}}^{2} \\
-a^{-1} P_{\mathrm{cr}, \mathrm{c}} \nabla_{x} \cdot \boldsymbol{u}_{\mathrm{A}}+\Gamma_{\mathrm{cr}, \mathrm{c}}+\Lambda_{\mathrm{cr}, \mathrm{c}} \\
\mathbf{0}
\end{array}\right)
\end{aligned}
$$

and we have assumed $\gamma_{\mathrm{cr}}=4 / 3$. This rescaling procedure minimises the appearance of cosmological source terms in the energy equation to a single term.

\subsection{Implementation}

To solve this coupled system of hyperbolic conservation laws in practice, we discretize quantities on a moving unstructured mesh defined by the Voronoi tessellation of a set of discrete points as realised in the AREPO code (Springel 2010). This numerical technique is known to cure numerical inaccuracies of smoothed particle hydrodynamics as well as adaptive mesh-refinement techniques (e.g., Springel 2010; Bauer \& Springel 2012; Sijacki et al. 2012).

We reconstruct the (comoving) primitive variables in the rest frame of a cell interface (with normal vector $\boldsymbol{n}$ ), using an improved second-order hydrodynamic scheme with Green-Gauss gradient estimates and a Runge-Kutta time integration (Pakmor et al. 2016a). We calculate the fluxes across the moving interface from the reconstructed primitive variables using the HLLD Riemann solver (Miyoshi \& Kusano 2005) as previously described (Pakmor et al. 2011). While the shear-Alfvén mode remains unaffected by a $\mathrm{CR}$ component, the magnitude of the fast and slow magnetoacoustic modes are modified according to

$$
\begin{aligned}
c_{\mathrm{f}, \mathrm{s}} & =\left[\frac{\gamma_{\mathrm{eff}} P+|\boldsymbol{B}|^{2} \pm \sqrt{\left(\gamma_{\mathrm{eff}} P+|\boldsymbol{B}|^{2}\right)^{2}-4 \gamma_{\mathrm{eff}} P B_{n}^{2}}}{2 \rho}\right]^{1 / 2} \\
\gamma_{\mathrm{eff}} P & =\gamma_{\mathrm{th}} P_{\mathrm{th}}+\gamma_{\mathrm{cr}} P_{\mathrm{cr}},
\end{aligned}
$$

where $B_{n}=\boldsymbol{B} \cdot \boldsymbol{n}$ (and equivalently for the cosmological analogue). We use the Powell scheme for divergence control (Powell et al. 1999) to evolve the (cosmological) MHD equations on our unstructured mesh (Pakmor \& Springel 2013).

To solve the CR energy equation, we passively advect $\varepsilon_{\mathrm{cr}}$ (or $\varepsilon_{\mathrm{cr}, \mathrm{c}}$ in cosmological simulations) on the modified Courant timestep $\Delta t_{\text {Cour }}=f_{\text {Cour }} \Delta x /\left(v_{\mathrm{m}}+c_{\mathrm{f}, \max }\right)$ with

$$
c_{\mathrm{f}, \max }=\left(\frac{\gamma_{\mathrm{eff}} P+|\boldsymbol{B}|^{2}}{\rho}\right)^{1 / 2},
$$

where $v_{\mathrm{m}}=\left|\boldsymbol{v}_{\mathrm{m}}\right|$ is the gas velocity in the frame of the 
mesh generating point, $\Delta x$ is the cell radius, and $f_{\text {Cour }}$ is the Courant factor. Note that this represents a conservative choice for the signal speed as $c_{\mathrm{f}, \max }$ is the maximum speed of the fast mode, which it acquires for propagating perpendicularly to the mean magnetic field.

The adiabatic source term $P_{\mathrm{cr}} \boldsymbol{\nabla} \cdot \boldsymbol{v}$ (or its comoving analogue) is calculated by employing Gauss' divergence theorem in every Voronoi cell and exchanging the corresponding fluxes across the interfaces to the neighbouring cells. Nonadiabatic CR source terms and active CR transport processes are treated in an operator-split fashion after evolving the homogeneous system by one time step. First, we account for sources of CR energy such as acceleration at resolved shocks or via a subgrid-scale model of injection at supernova remnants. We subsequently follow anisotropic CR diffusion. Finally, we cool the CR population by accounting for Coulomb and hadronic interactions. An implementation for CR streaming is left to future work.

Also note that we only provide the general formalism and simulations of advective CR transport in this paper. We defer a detailed exposition of a numerical algorithm that is capable of following anisotropic CR transport on an unstructured mesh to a companion paper (Pakmor et al. 2016b).

\section{NON-ADIABATIC PROCESSES}

In this section, we describe implementations for nonadiabatic processes that provide sources $\left(\Gamma_{\mathrm{cr}}\right)$ and sinks $\left(\Lambda_{\mathrm{cr}}\right)$ to the CR energy equation. The methods described here are for the acceleration of CRs either at shock fronts or injection via a subgrid treatment for supernova remnants and are for the cooling of CRs through Coulomb and hadronic processes.

\subsection{CR shock acceleration}

Diffusive shock acceleration is a universal process at collisionless shocks, which enables a small fraction of particles that impinge on the shock to gain substantially more energy than the average particle through multiple shock crossings. Provided that the shock propagates almost along the background magnetic field (quasi-parallel shock geometry with respect to the shock normal), it reforms quasi-periodically on ion cyclotron scales. Ions that enter the shock region when the discontinuity is the steepest are specularly reflected by the strong electrostatic shock potential and can be injected into the process of diffusive shock acceleration (Caprioli et al. 2015). In order to model the CR shock acceleration process in our hydro-dynamic simulations, we need to (i) find and characterise shocks within our simulation volume and (ii) model the CR acceleration with an efficiency that depends on shock Mach number and magnetic obliquity, i.e., the angle enclosed by the shock normal and upstream magnetic field orientation.

\subsubsection{Shock finding}

We employ the shock finding method developed by Schaal \& Springel (2015) and generalise it to include pre-existing as well as freshly accelerated CRs. Note that we restrict ourselves to the case without magnetic fields - an inclusion of those would add two more degrees of freedom to the system so that the resulting solution allows for two types of compressible shocks (slow-mode and fast-mode shocks) that are in principle able to accelerate particles. Moreover, efficient CR shock acceleration can amplify magnetic fields resonantly and non-resonantly (Caprioli \& Spitkovsky 2014b), further modifying the conservation laws.

A composite of thermal gas and CRs cannot be described by the equation of state of an ideal gas with an effective adiabatic index that is constant across the shock surface. Hence, the Rankine-Hugoniot shock jump conditions are necessarily modified from the the case of a single component fluid. Employing the continuity of mass and momentum across the shock in its rest frame (equation (B2) with $v_{\mathrm{s}}=0$ ), we can derive a closed form for the Mach number,

$$
\mathcal{M}_{1}^{2}=\frac{v_{1}^{2}}{c_{1}^{2}}=\left(\frac{P_{2}}{P_{1}}-1\right) \frac{x_{\mathrm{s}}}{\gamma_{\mathrm{eff}}\left(x_{\mathrm{s}}-1\right)} .
$$

Up- and downstream quantities are denoted by subscripts 1 and 2 , respectively, $x_{\mathrm{s}}=\rho_{2} / \rho_{1}$ is the density jump at the shock, $P_{i}=P_{\mathrm{th} i}+P_{\mathrm{cr} i}$ is the total pressure with $i \in\{1,2\}$, and $v_{1}$ denotes the velocity component parallel to the shock normal that is impinging on the shock from the upstream. The effective sound speed, $c_{1}$, and adiabatic index, $\gamma_{\mathrm{eff}}$, are given by

$$
\begin{gathered}
c_{1}^{2}=\frac{\gamma_{\mathrm{cr}} P_{\mathrm{cr} 1}+\gamma_{\mathrm{th}} P_{\mathrm{th} 1}}{\rho_{1}}=\frac{\gamma_{\mathrm{eff}} P_{1}}{\rho_{1}}, \text { and } \\
\left.\gamma_{\mathrm{eff}} \equiv \frac{\mathrm{d} \ln \left(P_{\mathrm{cr}}+P_{\mathrm{th}}\right)}{\mathrm{d} \ln \rho}\right|_{s}=\frac{\gamma_{\mathrm{cr}} P_{\mathrm{cr} 1}+\gamma_{\mathrm{th}} P_{\mathrm{th} 1}}{P_{1}} .
\end{gathered}
$$

Here the derivative is taken at constant generalised entropy $s$. Moreover, it proves beneficial to generalise the expression for the thermodynamic temperature by defining a pseudo temperature,

$$
k \tilde{T}=\frac{P}{n}=\frac{\mu_{\mathrm{mw}} m_{\mathrm{p}}\left(P_{\mathrm{th}}+P_{\mathrm{cr}}\right)}{\rho},
$$

where $n$ is the gas number density, $m_{\mathrm{p}}$ is the proton rest mass, and $\mu_{\mathrm{mw}}$ is the mean molecular weight.

We summarise the main steps of the generalised shock finding algorithm. First, we determine the direction of shock propagation in each Voronoi cell by identifying it with the unlimited gradient of the pseudo-temperature,

$$
d_{\mathrm{s}}=-\frac{\boldsymbol{\nabla} \tilde{T}}{|\boldsymbol{\nabla} \tilde{T}|} .
$$

The shock finding algorithm identifies a shock zone by applying the following local cell-based criteria to (i) find converging flows, (ii) filter spurious shocks such as tangential discontinuities and contacts, and (iii) providing a safeguard against labelling numerical noise as physical shocks:

(i) $\boldsymbol{\nabla} \cdot \boldsymbol{v}<0$,

(ii) $\boldsymbol{\nabla} \tilde{T} \cdot \boldsymbol{\nabla} \rho>0$,

(iii) $\tilde{\mathcal{M}}_{1}>\tilde{\mathcal{M}}_{\min }$.

We find that equation (29) is numerically not robust for spurious shocks or very weak shocks in combination with numerical noise. Thus, we employ the following, mathematically equivalent formulation to estimate the Mach number for the third criterion, using the density and pressure of 
neighbouring cells along the direction of shock propagation,

$\tilde{\mathcal{M}}_{1}^{2}= \begin{cases}\frac{1}{2 \gamma}\left[(\gamma+1) \frac{P_{2}}{P_{1}}+\gamma-1\right], & \text { for } \gamma_{1} \approx \gamma_{2} \equiv \gamma, \\ \frac{1}{\gamma_{\mathrm{eff}}} \frac{\left(y_{\mathrm{t}}-1\right) C}{C-\left[\left(\gamma_{1}+1\right)+\left(\gamma_{1}-1\right) y_{\mathrm{t}}\right]\left(\gamma_{2}-1\right)}, & \text { for } \gamma_{1} \neq \gamma_{2},\end{cases}$

where $C \equiv\left[\left(\gamma_{2}+1\right) y_{\mathrm{t}}+\gamma_{2}-1\right]\left(\gamma_{1}-1\right)$. Here, $y_{\mathrm{t}}=P_{2} / P_{1}$ denotes the total pressure jump across the interface of neighbouring cells and $\gamma_{i}=P_{i} / \varepsilon_{i}+1$, where $P_{i}=P_{\mathrm{th} i}+P_{\mathrm{cr} i}$ and $\varepsilon_{i}=\varepsilon_{\mathrm{th} i}+$ $\varepsilon_{\mathrm{cr} i}$ denote the total pressure and energy densities in the regions $i \in\{1,2\}$, respectively. The first line is a simplification provided $\gamma_{1}=\gamma_{2}$, and we use this expression if $2\left|\gamma_{1}-\gamma_{2}\right| /\left(\gamma_{1}+\right.$ $\left.\gamma_{2}\right)<0.01$. The tilde symbol indicates that $\tilde{\mathcal{M}}_{1}$ is only a lower limit to the true Mach number if the shock is broadened over more than the neighbouring cells. For concreteness, we chose a minimum Mach number $\tilde{\mathcal{M}}_{\min }=1.3$. We note that the combination of adiabatic indexes $\gamma_{1}, \gamma_{2}$, and $\gamma_{\text {eff }}$ of the previous equation are related by the shock adiabat and are not independent variables. As a result, the Mach number has to obey the consistency relation (which derives from the requirement that the pressure jump is a real quantity),

$$
\tilde{\mathcal{M}}_{1}^{2}>\frac{1}{\gamma_{\mathrm{eff}}}\left[\frac{\gamma_{2}^{2}-\gamma_{1}}{\gamma_{1}-1}+\sqrt{\left(\frac{\gamma_{2}^{2}-\gamma_{1}}{\gamma_{1}-1}\right)^{2}-\gamma_{2}^{2}}\right] .
$$

We tag cells that are inside the shock zone and show a maximum compression value along the direction of shock propagation $\boldsymbol{d}_{\mathrm{s}}$ as shock surface cells. In walking along the ray that is oriented along $\boldsymbol{d}_{\mathrm{s}}$ from the shock surface to the 'true' post-shock state, we collect the total energy $E_{\mathrm{tot}, i}=E_{\mathrm{th}, i}+E_{\mathrm{cr}, i}$ of each of those cells $i$, which represent the numerically broadened post-shock states. After reaching the post-shock state (which represents the first cell outside the shock zone), we reverse the direction of the ray and pass through the entire shock zone, which has a typical thickness of 3 to 4 cells, to determine the pre-shock energy $E_{\text {tot, } 1}$. This will be needed later on for modelling CR shock acceleration. The Mach number for a shock surface cell is calculated with equation (29) across the shock zone by using the preand post-shock states in the cells directly adjacent to the shock zone. Finally, we account for overlapping shock zones which can be present in colliding shocks (for details, please see Schaal \& Springel 2015).

\subsubsection{Diffusive shock acceleration of CRs}

A shock dissipates kinetic energy into thermal and nonthermal energy with a corresponding dissipated energy density that is equal to the total post-shock energy density corrected for the adiabatically compressed pre-shock energy density,

$$
\varepsilon_{\mathrm{diss}}=\varepsilon_{\mathrm{th} 2}+\varepsilon_{\mathrm{cr} 2}-\varepsilon_{\mathrm{th}, 1} x_{\mathrm{s}}^{\gamma_{\mathrm{th}}}-\varepsilon_{\mathrm{cr}, 1} x_{\mathrm{s}}^{\gamma_{\mathrm{cr}}},
$$

where $\varepsilon_{\mathrm{cr} 2}$ attains contributions from the adiabatically compressed pre-existing CRs and the freshly accelerated CRs. The dissipated energy flux at a shock ( $f_{\text {diss }}$ in units of energy per unit area and per unit time) is given by the dissipated energy density times the post-shock velocity $\left(v_{2}\right)$,

$$
\begin{aligned}
& f_{\text {diss }}=\varepsilon_{\text {diss }} v_{2}=\varepsilon_{\text {diss }} \frac{\mathcal{M}_{1} c_{1}}{x_{\mathrm{s}}}, \text { so that } \\
& \dot{E}_{\text {diss }}=f_{\text {diss }} A_{\text {shock }}
\end{aligned}
$$

is the shock-dissipated energy per unit time and $A_{\text {shock }}$ the area of the shock surface within a shocked cell.

Diffusive shock acceleration is able to convert a fraction $\zeta$ of the shock-dissipated energy to CRs,

$$
\Delta E_{\mathrm{cr}}=\zeta\left(\mathcal{M}_{1}, \theta\right) E_{\mathrm{diss}},
$$

where $E_{\text {diss }}=\dot{E}_{\text {diss }} \Delta t$ in our simulations with a discretized time integration scheme. As indicated, the CR acceleration efficiency depends on the shock strength as given by the Mach number $\mathcal{M}_{1}$, the magnetic obliquity $\theta$, and perhaps the plasma beta parameter. In practice, we define the magnetic obliquity as the angle between the shock normal and the magnetic field direction in the shock surface cell. We adopt an acceleration efficiency that is determined from recent hybrid particle-in-cell (PIC) simulations with kinetic protons and a charge neutralising electron fluid (Caprioli \& Spitkovsky 2014a). Provided the magnetic obliquity $\theta \lesssim 45^{\circ}$ (quasi-parallel geometry) and $\mathcal{M}_{1} \geqslant \mathcal{M}_{\text {crit }}$, the CR acceleration efficiency is $\zeta \approx 0.1 .^{3}$ Depending on the exact physics that determines $\mathrm{CR}$ injection into the diffusive shock acceleration process, we typically use $\mathcal{M}_{\text {crit }} \approx 3 .{ }^{4}$

We inject CR energy into the shock surface cells (labelled with a subscript $s$ ) as well as into the numerically broadened post-shock cells $i$ (including the 'true' post-shock cell, i.e., the first cell outside the 'shock zone') according to the following prescription,

$$
\begin{aligned}
\Delta E_{\mathrm{tot}} & =\sum_{j=s}^{s+n}\left(E_{\mathrm{tot}, j}-E_{\mathrm{tot}, 1}\right) \\
\Delta E_{\mathrm{cr}, i} & =\zeta\left(\mathcal{M}_{1}, \theta\right) E_{\mathrm{diss}} \frac{E_{\mathrm{tot}, i}-E_{\mathrm{tot}, 1}}{\Delta E_{\mathrm{tot}}} .
\end{aligned}
$$

Here, $E_{\mathrm{tot}, 1}$ is the pre-shock energy, $E_{\mathrm{tot}, i}=\left(\varepsilon_{\mathrm{th}, i}+\varepsilon_{\mathrm{cr}, i}\right) V_{i}$, and $V_{i}$ is the volume of a Voronoi cell $i$. This sum typically extends over $n=2$ to 3 cells. This prescription neglects the adiabatically compressed thermal and CR energies over the shock, which are insignificant for strong shocks that efficiently accelerate CRs. We found that this prescription yields robust results in a uniform medium as well as in cases where the shock propagates into a steeply stratified atmosphere.

\subsection{CR injection at supernova remnants}

Supernova explosions drive strong shocks into the ISM, which are believed to be one of the most important sources of the galactic CRs up to PeV energies. In our global (cosmological) galaxy formation models, we cannot afford to resolve individual supernova remnants (unlike in our companion paper, Simpson et al. 2016). Hence, we need to resort to a subresolution treatment of star formation and its regulation by supernova remnants. To compute radiative cooling in the galaxy formation simulations presented here, we assume an

3 The acceleration efficiency in those PIC simulations is defined to be the energy density fraction contained in non-thermal particles and was determined in the post-shock rest frame, which by definition has no kinetic contribution to the energy. This coincides with our definition provided we consider strong shocks that have a negligible pre-shock thermal energy density.

${ }^{4}$ We defer a detailed study of how the magnetic obliquity dependence of the acceleration efficiency influences the MHD solution to future work. 
optically thin gas in collisional ionisation equilibrium and include heating by a photo-ionising, time-dependent, spatially uniform ultraviolet background. Star formation and thermal supernovae feedback are modelled using the hybrid multiphase model for the interstellar medium of Springel \& Hernquist (2003). In this model, star formation is treated stochastically and the star formation rate is correlated to the free-fall time of self-gravitating sheets of gas, according to the observed Kennicutt law (1998).

In the original model, the energy released by supernovae heats the ambient hot phase of the ISM and evaporates clouds in supernova remnants. These effects establish a tightly self-regulated regime for star formation in the ISM. In practice, the ISM is described by a sub-resolution model, which assumes the ISM to be pressurised by star formation feedback so that there exists a coexistence between the hot phase and embedded cold clouds in pressure equilibrium. This is parametrized by a stiff effective equation of state above a critical density that sets the star formation threshold, $\rho_{0} \simeq 4 \times 10^{-25} \mathrm{~g} \mathrm{~cm}^{-3}$, above which the model interpolates between the hot and cold phases.

In order to model CR injection ${ }^{5}$ at supernova remnants, we assume that an energy fraction $\zeta_{\mathrm{SN}}=0.1$ of the supernova energy can be transferred to a CR population (Helder et al. 2012; Morlino \& Caprioli 2012; Ackermann \& et al. 2013). So far, we only model CR injection at core-collapse supernovae and leave the modelling of type Ia supernovae for future work. The total energy injection rate by supernovae for a given star formation rate density $\dot{\rho}_{\star}$ depends on the initial mass function (IMF). Assuming a Kroupa IMF (Kroupa 2001) and that stars above a mass of $8 \mathrm{M}_{\odot}$ explode as supernovae, we find a mass fraction of 0.128 of stars that end up in supernovae, and about one supernova per $100 \mathrm{M}_{\odot}$ of stellar mass formed.

With a canonical kinetic energy release of $10^{51}$ erg per supernova, this translates to an energy injection rate per unit volume of $\epsilon_{\mathrm{SN}} \dot{\rho}_{\star}$ with $\epsilon_{\mathrm{SN}}=10^{49} \mathrm{erg} \mathrm{M}_{\odot}^{-1}$. We model the $\mathrm{CR}$ energy injection per timestep of a gas cell as,

$$
\Delta E_{\mathrm{cr}}=\zeta_{\mathrm{SN}} \epsilon_{\mathrm{SN}} \dot{m}_{\star} \Delta t,
$$

where $\dot{m}_{\star}=m_{\star} \dot{\rho}_{\star} / \rho$ is the star formation rate of the mesh cell. The uncertainties in any of the parameters are parametrized with $\zeta_{\mathrm{SN}}$, which controls the amount of CR energy that is generated by supernovae. We inject this energy into the local environment of every newly created star particle using a spherical top hat filter that contains the closest 32 mesh cells of the star. The remaining supernova energy is assumed to be transferred to subgrid-scale turbulence that dissipates and heats the ambient gas, thereby establishing a tightly self-regulated regime of the ISM.

\subsection{Collisional loss processes of CRs}

CR particles propagating through a plasma will gradually dissipate their kinetic energy, and transfer it to the surrounding thermal plasma through individual electron scatterings in the Coulomb field of the CR particle as well as via

5 Note that we use the terminology ' $\mathrm{CR}$ injection' for a one-time $\mathrm{CR}$ energy gain whereas we adopt the phrase 'CR acceleration' for a continuous acceleration process at resolved shocks. small momentum transfers through excitations of quantised plasma oscillations. We refer to the sum of both effects as Coulomb losses. Additionally, energetic CRs collide inelastically with ambient thermal gas to produce pions, causing a catastrophic energy loss for the CRs. This process we refer to as hadronic losses. The rate of these energy loss mechanisms depends both on the physical properties of the ambient medium and on the detailed momentum spectrum of the $\mathrm{CR}$ population.

Hadronic interactions only affect the high-momentum regime with a rate that is to good approximation independent of momentum so that the spectral shapes remains largely invariant. In contrast, an accurate determination of the Coulomb loss rate of a CR population would require a full dynamical modelling of the CR momentum spectrum. Particles with low momenta are most strongly affected by Coulomb interactions with a rate that increases towards lower momenta. As a consequence, this induces a spectral break of the CR momentum distribution: while the highmomentum tail remains invariant, the low-momentum CR particles transfer their energy effectively to the thermal gas, and eventually get thermalized. Since we are not following the momentum distribution in this work, we will construct an equilibrium momentum distribution that balances $\mathrm{CR}$ injection on the one side and CR losses on the other side. The $\mathrm{CR}$ cooling and thermal heating rates are then derived from this equilibrium distribution, which is valid on timescales long compared to impulsive changes in the injection process.

\subsubsection{Coulomb losses}

The kinetic energy of a proton with dimensionless momentum $p=P_{\mathrm{p}} /\left(m_{\mathrm{p}} c\right)$ is given by

$$
E_{\mathrm{p}}(p)=\left(\sqrt{1+p^{2}}-1\right) m_{\mathrm{p}} c^{2} .
$$

The kinetic energy loss of a proton with $\gamma \ll m_{\mathrm{p}} / m_{\mathrm{e}}$ due to Coulomb interactions in a plasma is (Gould 1972)

$$
-\left(\frac{\mathrm{d} E_{\mathrm{p}}(p)}{\mathrm{d} t}\right)_{\text {Coul }}=\frac{4 \pi e^{4} n_{\mathrm{e}}}{m_{\mathrm{e}} \beta c}\left[\ln \left(\frac{2 m_{\mathrm{e}} c^{2} \beta p}{\hbar \omega_{\mathrm{pl}}}\right)-\frac{\beta^{2}}{2}\right] \equiv \frac{A_{\text {Coul }}}{\beta} .
$$

Here, $\omega_{\mathrm{pl}}=\sqrt{4 \pi e^{2} n_{\mathrm{e}} / m_{\mathrm{e}}}$ is the plasma frequency, $n_{\mathrm{e}}$ is the number density of free electrons, and $\beta \equiv v_{\mathrm{p}} / c=p / \sqrt{1+p^{2}}$ is the dimensionless $\mathrm{CR}$ velocity. The energy loss due to Coulomb interactions in a neutral gas can also be approximately calculated with this formula, provided $n_{\mathrm{e}}$ is taken to be the total electron number density (free plus bound). Note that atomic charge shielding effects lower the actual Coulomb loss rate so that a more accurate description of ionisation losses is desirable (Enßlin et al. 2007). To obtain an approximate analytical expression for the CR equilibrium distribution, we replace the term $\beta p$ in the Coulomb logarithm with its mean value for the spectrum, which can be written as $\langle\beta p\rangle=3 P_{\mathrm{cr}} /\left(m_{\mathrm{p}} c^{2} n_{\mathrm{cr}}\right)$. We also define a CR cooling timescale due to Coulomb cooling as

$$
\tau_{\text {Coul }} \equiv \frac{\varepsilon_{\mathrm{cr}}}{\left|\mathrm{d} \varepsilon_{\mathrm{cr}} / \mathrm{d} t\right|_{\text {Coul }}} .
$$

\subsubsection{Hadronic losses}

CR protons interact inelastically with nuclei of the surrounding thermal gas and produce mainly pions, provided 
their momentum exceeds the kinematic threshold $p_{\text {thr }} m_{\mathrm{p}} c=$ $0.78 \mathrm{GeV} / c$ for the hadronic reaction. The neutral pions decay after a mean lifetime of $9 \times 10^{-17} \mathrm{~s}$ into $\gamma$-rays, and the charged pions decay into secondary electrons/positrons and neutrinos:

$$
\begin{aligned}
\pi^{ \pm} & \rightarrow \mu^{ \pm}+v_{\mu} / \bar{v}_{\mu} \rightarrow e^{ \pm}+v_{e} / \bar{v}_{e}+v_{\mu}+\bar{v}_{\mu} \\
\pi^{0} & \rightarrow 2 \gamma .
\end{aligned}
$$

The presence of CRs can be uniquely identified by the characteristic spectral signature in $\gamma$-rays, the pion-decay bump, which is centred on half the pion's rest mass in the differential spectrum, as well as by the neutrino emission. The luminosity of secondary electrons/positrons produced in hadronic interactions amounts to a fraction of $1 / 6$ of that of the total pion luminosity (using isospin symmetry, there is a branching ratio of $2 / 3$ to produce charged pions and the mean energies of the produced secondaries in the laboratory frame is $\left.\left\langle E_{e^{ \pm}}\right\rangle=\left\langle E_{\pi^{ \pm}}\right\rangle / 4\right)$. Those secondaries cool via synchrotron emission and Compton scattering off photons of any radiation field (cosmic microwave background or star light) but the associated emission signatures are not unique due to the presence of other possible relativistic electron distributions.

The hadronic energy loss of a CR proton is independent of the partitioning among the pions during hadronic interactions and given by

$$
\begin{aligned}
-\left(\frac{\mathrm{d} E_{\mathrm{p}}}{\mathrm{d} t}\right)_{\text {hadr }} & =n_{\mathrm{N}} \sigma_{\mathrm{pp}} K_{\mathrm{p}} m_{\mathrm{p}} c^{3}(\gamma-1) \theta\left(p-p_{\mathrm{thr}}\right) \\
& \equiv A_{\text {hadr }}(\gamma-1) \theta\left(p-p_{\text {thr }}\right) .
\end{aligned}
$$

Here, $\sigma_{\mathrm{pp}} \approx 44.2$ mbarn is the total pion cross section (Pfrommer \& Enßlin 2004, assuming that the one-dimensional $\mathrm{CR}$ distribution function has a momentum spectral index $\alpha=2.2), K_{\mathrm{p}} \approx 1 / 2$ denotes the inelasticity of the hadronic reaction in the limiting regime (Mannheim \& Schlickeiser 1994), and $n_{\mathrm{N}}=n_{\mathrm{e}} /\left(1-X_{\mathrm{He}} / 2\right)$ is the target nucleon density in the ICM, assuming primordial element composition with $X_{\mathrm{He}}=0.24$. By analogy with Coulomb interactions, we define a CR cooling timescale owing to hadronic interactions as

$$
\tau_{\text {hadr }} \equiv \frac{\varepsilon_{\text {cr }}}{\left|\mathrm{d} \varepsilon_{\text {cr }} / \mathrm{d} t\right|_{\text {hadr }}} .
$$

\subsubsection{Equilibrium distribution}

To estimate the total loss rate due to Coulomb and hadronic reactions, we will derive an equilibrium spectrum which balances continuous CR injection and the described energy losses (following Enßlin et al. 2007). Assuming a homogeneous environment and an isotropic CR distribution in momentum space, the effective one-dimensional CR spec$\operatorname{trum} f_{\mathrm{p}}^{(1)}(p, t)=4 \pi p^{2} f_{\mathrm{p}}(p, t)$ is governed by the Fokker-Planck equation,

$$
\frac{\partial f_{\mathrm{p}}^{(1)}(p, t)}{\partial t}+\frac{\partial}{\partial p}\left[\dot{p}(p, t) f_{\mathrm{p}}^{(1)}(p, t)\right]=Q^{(1)}(p)-\frac{f_{\mathrm{p}}^{(1)}(p, t)}{\tau_{\text {loss }}(p)},
$$

where the momentum loss rates due to Coulomb and hadronic interactions are

$$
\dot{p}(p)=\left[\left(\frac{\mathrm{d} E_{\mathrm{p}}(p)}{\mathrm{d} t}\right)_{\text {Coul }}+\left(\frac{\mathrm{d} E_{\mathrm{p}}}{\mathrm{d} t}\right)_{\text {hadr }}\right]\left(\frac{\mathrm{d} E_{\mathrm{p}}(p)}{\mathrm{d} p}\right)^{-1} .
$$

We approximate hadronic losses as continuously occurring rather than catastrophically, assume a sufficiently extended environment so that we can ignore escape from the system $\left(\tau_{\text {loss }} \rightarrow \infty\right)$, and search for steady state solutions $\left(\partial f^{(1)} / \partial t=0\right)$. We assume that the CR injection spectrum is a power-law in momentum,

$$
Q^{(1)}(p)=Q_{\text {inj }} p^{-\alpha_{\text {inj }}} \theta\left(p-p_{1}\right),
$$

where $\theta(x)$ is the Heaviside distribution, which is unity for positive arguments and zero otherwise, and $p_{1}$ is the low-momentum cutoff. Using the Jacobian transformation $\mathrm{d} E_{\mathrm{p}}(p) / \mathrm{d} p=m_{\mathrm{p}} c^{2} \beta(p)$, we find the asymptotic steady state spectrum by assuming negligible hadronic and Coulomb losses in the low- and high-momentum regimes, respectively,

$$
f_{\mathrm{p}}^{(1)}(p)=\frac{Q_{\mathrm{inj}} p^{-\alpha_{\mathrm{inj}}}}{\left(\alpha_{\mathrm{inj}}-1\right) A_{\mathrm{Coul}}} \times \begin{cases}p^{3}, & p_{1} \ll p \ll p_{*}, \\ p_{*}^{3}, & p \gg p_{*} .\end{cases}
$$

The intersection momentum $p_{*}$ depends on the ratio of the Coulomb-to-hadronic loss rates

$$
p_{*}=\sqrt[3]{\frac{A_{\mathrm{Coul}}}{A_{\mathrm{hadr}}}}=\sqrt[3]{\frac{4 \pi e^{4} n_{\mathrm{e}}}{m_{\mathrm{e}} m_{p} c^{4} \sigma_{\mathrm{pp}} K_{\mathrm{p}} n_{\mathrm{N}}} \ln \left(\frac{2 m_{\mathrm{e}} c^{2}\langle\beta p\rangle}{\hbar \omega_{\mathrm{pl}}}\right)} \approx 1.1,
$$

where we have dropped the small term due to momentum transfers through excitations of quantised plasma oscillations in the Coulomb cooling rate. The numerical value of $p_{*} \approx 1.1$ is accurate to 10 per cent for electron densities in the range $\left[10^{-6}, 10^{3}\right] \mathrm{cm}^{-3}$ (Enßlin et al. 2007).

We provide an analytic approximation to the steady state equilibrium $\mathrm{CR}$ spectrum and introduce a matched asymptotic solution,

$$
f_{\mathrm{p}}^{(1)}(p)=\frac{Q_{\text {inj }} p^{-\alpha_{\text {inj }}} \theta\left(p-p_{1}\right)}{\left(\alpha_{\text {inj }}-1\right) A_{\text {Coul }}\left(p_{*}^{-3}+p^{-3}\right)} .
$$

Using this approximate steady state spectrum, we derive the Coulomb loss rate of a CR population,

$$
\begin{aligned}
\Lambda_{\text {Coul }} & =\int_{p_{1}}^{\infty} f_{\mathrm{p}}^{(1)}(p)\left(\frac{\mathrm{d} E_{\mathrm{p}}}{\mathrm{d} t}\right)_{\text {Coul }} \mathrm{d} p \\
& =-2.78 \times 10^{-16}\left(\frac{n_{\mathrm{e}}}{\mathrm{cm}^{-3}}\right)\left(\frac{\varepsilon_{\mathrm{cr}}}{\mathrm{erg} \mathrm{cm}^{-3}}\right) \mathrm{erg} \mathrm{s}^{-1} \mathrm{~cm}^{-3},
\end{aligned}
$$

where we expressed the normalisation of the injection spectrum $Q_{\mathrm{inj}}$ in terms of the CR energy density $\varepsilon_{\mathrm{cr}}$ and we adopted $p_{1}=0.1$ and $\alpha_{\text {inj }}=2.2$, which is characteristic of the CR injection spectrum at supernova remnants or jets of active galactic nuclei. The loss rate $\Lambda_{\text {Coul }}$ depends somewhat on the value of $p_{1}$, but only weakly as the low-momentum part attains a weak momentum scaling, $p^{3-\alpha_{\text {inj }}}$. It does, however, depend on the precise value of $\alpha_{\mathrm{inj}}$ and varies in the range $\Lambda_{\text {Coul }}=-1.35 \times 10^{-16}$ to $-12.8 \times 10^{-16} \mathrm{erg} \mathrm{s}^{-1} \mathrm{~cm}^{-3}$ for $\alpha_{\text {inj }}=2.1$ to 2.7. Similarly, the hadronic loss rate of an equilibrium CR population is

$$
\begin{aligned}
\Lambda_{\text {hadr }} & =\int_{p_{1}}^{\infty} f_{\mathrm{p}}^{(1)}(p)\left(\frac{\mathrm{d} E_{\mathrm{p}}}{\mathrm{d} t}\right)_{\text {hadr }} \mathrm{d} p \\
& =-7.44 \times 10^{-16}\left(\frac{n_{\mathrm{e}}}{\mathrm{cm}^{-3}}\right)\left(\frac{\varepsilon_{\mathrm{cr}}}{\mathrm{erg} \mathrm{cm}^{-3}}\right) \mathrm{erg} \mathrm{s}^{-1} \mathrm{~cm}^{-3} .
\end{aligned}
$$

The hadronic loss rate depends less sensitively on the precise value of $\alpha_{\text {inj }}$; it varies as $\Lambda_{\text {hadr }}=-8.1 \times 10^{-16}$ to $-5.6 \times$ $10^{-16} \mathrm{erg} \mathrm{s}^{-1} \mathrm{~cm}^{-3}$ for $\alpha_{\text {inj }}=2.1$ to 2.7. While the Coulomb loss rate is sub-dominant for hard injection indexes $\alpha_{\text {inj }} \lesssim 2.4$, it dominates over hadronic losses for softer injection spectra. 
The total CR energy-loss rate due to Coulomb and hadronic interactions is $\Lambda_{\mathrm{cr}}=\Lambda_{\mathrm{hadr}}+\Lambda_{\text {Coul }}=-\lambda_{\mathrm{cr}} n_{\mathrm{e}} \varepsilon_{\mathrm{cr}}$ where $\lambda_{\mathrm{cr}}=1.022 \times 10^{-15} \mathrm{~cm}^{3} \mathrm{~s}^{-1}$ is the rate coefficient for collisional energy loss of the CRs. This allows us to implicitly follow the associated energy loss of CRs according to the solution

$$
E_{\text {cr }}(t)=E_{\text {cr }}\left(t_{0}\right) \mathrm{e}^{-\lambda_{\text {cr }} n_{\mathrm{e}}} .
$$

The CR energy lost in Coulomb interactions is entirely thermalized, thereby heating the surrounding plasma of the ISM or intracluster medium. In contrast, most of the hadronic energy losses escape in $\gamma$ rays and neutrinos as the interaction regions are optically thin to these hadronic decay products.

As explained in Sect. 3.3.2, on average a fraction of $1 / 6$ of the hadronically dissipated luminosity ends up in secondary electrons/positrons. While the highly energetic electrons $\left(\gamma_{\mathrm{e}} \gtrsim 10^{3}\right)$ cool via synchrotron emission as well as Compton scattering, most of the mildly relativistic electrons $\left(\gamma_{\mathrm{e}} \lesssim 200\right)$ will thermalize this energy via Coulomb collisions (Rephaeli 1979; Sarazin 1999). The secondary electrons generated at the kinematic threshold of the hadronic reaction have a mean energy of $\left\langle E_{\mathrm{e}^{ \pm}}\right\rangle=m_{\pi^{ \pm}} c^{2} / 4=35 \mathrm{MeV}=68 m_{\mathrm{e}} c^{2}$. Hence, we assume that the majority of the energy of secondary electrons/positrons is used for heating the surrounding plasma by Coulomb interactions. As a result, the collisional heating rate due to Coulomb and hadronic interactions is given by $\Gamma_{\text {th }}=-\Lambda_{\text {Coul }}-\Lambda_{\text {hadr }} / 6=\lambda_{\text {th }} n_{\mathrm{e}} \varepsilon_{\mathrm{cr}}$, where $\lambda_{\text {th }}=4.02 \times 10^{-16} \mathrm{~cm}^{3} \mathrm{~s}^{-1}$ is the rate coefficient for collisional heating of the ambient plasma by CRs. We obtain a gain of thermal energy of

$$
\Delta E_{\mathrm{th}}=E_{\mathrm{cr}}\left(1-\mathrm{e}^{-\lambda_{\mathrm{th}} n_{\mathrm{e}} t}\right) .
$$

due to these collisional CR heating processes.

\subsubsection{Limitations}

What are the conditions of validity for our approach of computing the CR loss rate from the equilibrium distribution? Clearly, this is an excellent description if CR injection balances the non-adiabatic cooling processes. As we will show in the following, this equilibrium distribution also provides a good approximation to the case of a freely cooling CR population in the absence of injection at late times. While Coulomb cooling increases the spectral break, catastrophic $\mathrm{CR}$ losses due to hadronic interactions lowers the break. As a result, the CR population evolves towards an attractor solution that resembles the equilibrium distribution and exhibits an identical spectral break, but shows a low-momentum spectrum with a modified power-law index (see figures 5 and 6 in Enßlin et al. 2007).

In general, a freely cooling CR spectrum has to be integrated numerically, but we can analytically demonstrate this effect for a simplified case of a CR power-law distribution,

$$
f(p)=C p^{-\alpha} \theta(p-q) .
$$

Non-adiabatic cooling processes lead to a change of the energy density, $\mathrm{d} \varepsilon_{\mathrm{cr}}=\left(\tau_{\text {hadr }}^{-1}+\tau_{\text {Coul }}^{-1}\right) \varepsilon_{\mathrm{cr}} \mathrm{d} t$ and a change of the number density $\mathrm{d} n_{\mathrm{cr}}=\varepsilon_{\mathrm{cr}} \mathrm{d} t /\left[\tau_{\text {Coul }} E_{\mathrm{p}}(q)\right]$ because hadronic interactions conserve the number of CRs. The implied change in the normalisation $C$ and low-momentum cutoff $q$ can be straightforwardly calculated by means of a Jacobian transformation. Hence, at late times Coulomb and hadronic interactions reach a balance with fixed cutoff $q_{\text {fix }}$, which is given as a solution of the equation (Jubelgas et al. 2008)

$$
\frac{T_{\mathrm{cr}}\left(\alpha, q_{\mathrm{fix}}\right)}{E_{\mathrm{p}}\left(q_{\mathrm{fix}}\right)}=1+\frac{\tau_{\text {Coul }}\left(q_{\mathrm{fix}}\right)}{\tau_{\text {hadr }}\left(q_{\mathrm{fix}}\right)},
$$

where $T_{\text {cr }}=\varepsilon_{\text {cr }} / n_{\text {cr }}$ is the average kinetic energy of a CR population. The normalisation decreases with time as

$$
\frac{\mathrm{d} \ln C}{\mathrm{~d} t}=\left[\tau_{\text {hadr }}(q)\left(1-\frac{E_{\mathrm{p}}(q)}{T_{\mathrm{cr}}(\alpha, q)}\right)\right]^{-1} \rightarrow \frac{\tau_{\mathrm{hadr}}+\tau_{\mathrm{Coul}}}{\tau_{\mathrm{hadr}} \tau_{\mathrm{Coul}}},
$$

for $q \rightarrow q_{\mathrm{fix}}$. This shows that a freely cooling CR distribution has an invariant spectral shape at late times and decays with a constant rate that is given by the right-hand side of equation (59).

However, this approach is potentially problematic if the real CR spectrum is not in equilibrium. This is particularly true after a fresh CR injection event, in which case Coulomb losses should efficiently thermalize the low-momentum part of the CR distribution. Instead, Coulomb cooling of our equilibrium spectrum only removes CRs at low (late-time) cooling rates. The net result is an initially substantially slower Coulomb cooling than a full (non-equilibrium) solution with a time-dependent spectrum would provide. We defer a detailed study, which follows the CR spectrum in time and space, to future work.

\section{RESULTS}

All simulations and application runs discussed in the following section were performed with the moving mesh set-up of AREPO using standard parameters for mesh regularisation (Vogelsberger et al. 2012; Pakmor et al. 2016a). We also repeated some of these simulations with a spatially fixed mesh and report any significant differences to the moving mesh case.

\subsection{Shock tubes}

To assess the validity of our shock acceleration algorithm and to gain confidence in our numerical CR implementation, we perform a sequence of shock-tube simulations in one, two, and three dimensions, with a range of shock strengths, and with various resolutions. In Fig. 1, we present three types of problem set-ups: a shock tube that encounters a pre-existing population of CRs, with and without taking into account CR acceleration, as well as a shock propagating in thermal gas with inlined CR acceleration. Note that since this problem is scale-free, the solution can be scaled to any astrophysical problem at hand.

Solutions of the Riemann shock-tube problem exist in the case of a single polytropic fluid (Courant \& Friedrichs 1948) as well as for a two-component fluid composed of CRs and thermal gas (Pfrommer et al. 2006), in which the CRs are adiabatically compressed over the shock. So far, an exact solution that also accounts for CR shock acceleration has not been reported in the literature to our knowledge. To close this gap, we derive such an exact solution of a shock tube with CR shock acceleration in the case of a shock propagating in purely thermal gas (Appendix B) as well as for the case of a shock propagating in a composite of CRs and thermal gas (Appendix C).

Figure 1 shows the resulting shock-tube setup for our 

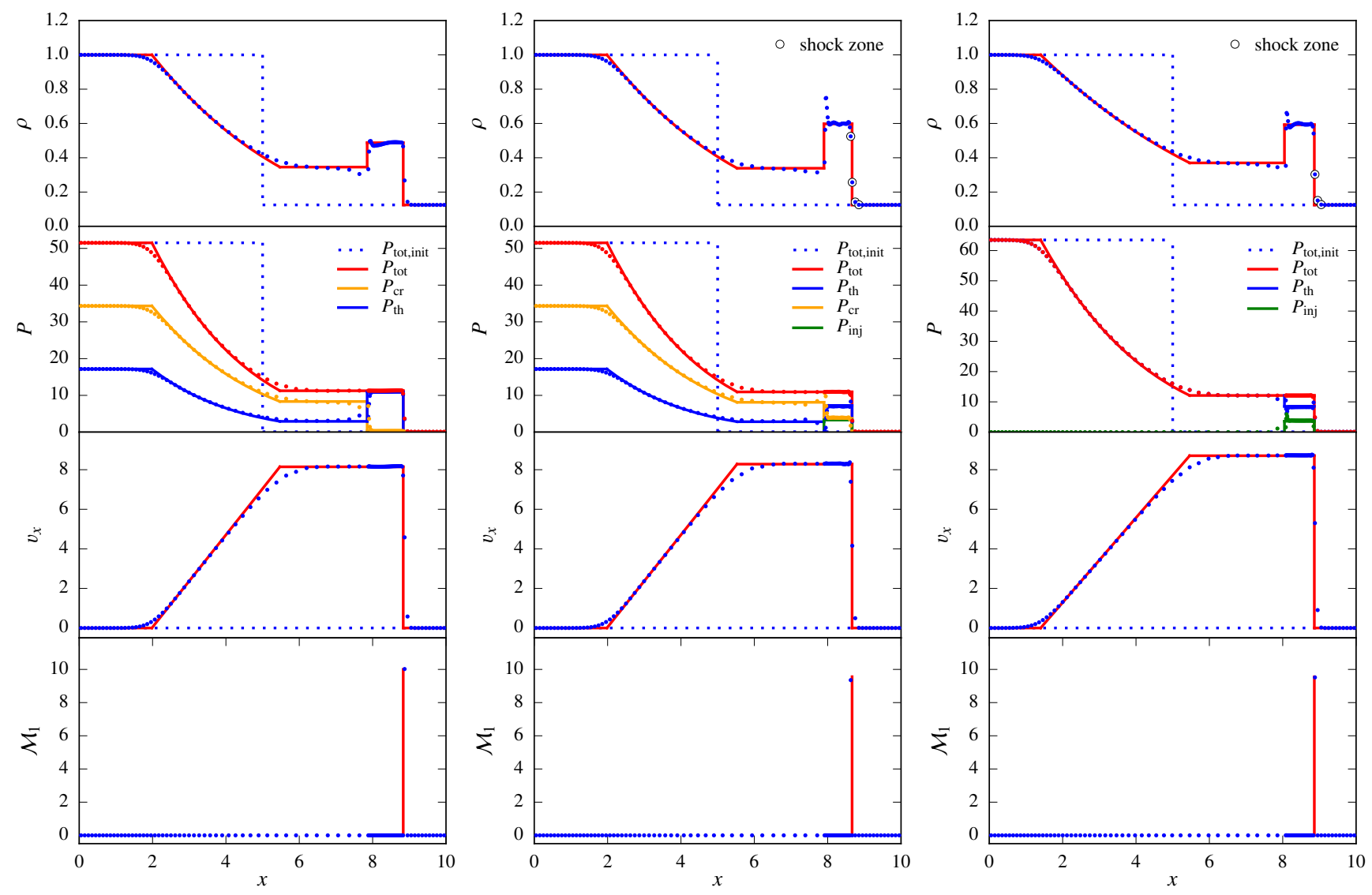

Figure 1. CR shock-tube tests of our moving mesh code. Shown are one-dimensional simulations with 100 cells at time $t=0.35$. In each of the three columns, we show from the top to bottom: mass density $\rho$, pressure $P$, velocity $v_{x}$, and pre-shock Mach number $\mathcal{M}_{1}$ of our simulations (points) and the exact solution (solid). The initial state of the Riemann problem is also shown (blue dotted). Left-hand panels: the shock propagates through a composite of CRs and thermal gas without taking into account CR acceleration. Initially, the CR pressure is twice the thermal pressure in the left-half space and equal to the thermal pressure in the right-half space. Upon encountering the shock, the CRs are only adiabatically compressed. Middle panels: Same initial conditions as before, but now also accounting for CR shock acceleration $\left(P_{\text {inj }}\right.$ shown in green). Shown is also the shock zone (open circles) and the shock surface (cell with $\left.\mathcal{M}_{1} \neq 0\right)$. As a result of $\mathrm{CR}$ acceleration, the post-shock gas is more compressible and denser. Thus, the shock does not propagate as fast as in the case without CR acceleration. Right-hand panels: this shock tube shows CR-shock acceleration in a thermal gas without pre-existing CRs.

one-dimensional simulations with 100 mesh cells that are initially equally spaced. We choose our initial conditions such that a shock with Mach number of $\mathcal{M}_{1}=10$ forms in the absence of CR acceleration. Any deviation of that is an immediate consequence of CR acceleration. The detailed simulation set-up is reported in Table 1. To examine our CR shock-injection algorithm at the extreme, we choose a high value for the $\mathrm{CR}$ acceleration efficiency of $\zeta=0.5$ and do not account for non-adiabatic CR loss processes.

Overall, there is excellent agreement of our simulations with the exact solutions. This agreement implies an excellent performance of the approximate HLLD Riemann solver for the two-fluid problem of thermal gas and CRs that our exact solution adopts. We note that the averaged hydrodynamic values in our two- and three-dimensional shock tube simulations match the analytic solutions similarly well. To understand how our CR injection algorithm works in practice, we also show the shock zone (Section 3.1.1). The shock surface, i.e., the cell within the shock zone that has the highest velocity divergence, characterised by $\mathcal{M}_{1} \neq 0$, is almost always situated at the edge of the shock zone. This result holds also for weaker shock waves. It implies that our algorithm injects CRs mostly into two cells, the shock surface and the first cell outside the shock zone, in the direction towards the post-shock region. Injecting and accelerating CRs from the thermal pool lowers the effective adiabatic index of the post-shock gas, making it more compressible and thus denser. As a result of this and as a consequence of mass conservation, the shock does not propagate as fast as in the case without $\mathrm{CR}$ acceleration.

The only noticeable difference to the exact solution is the high-density blip in the first two cells past the contact discontinuity. This comes about because in the first few time steps after the start of the simulation when the shock has not yet fully developed and the post-shock regime is about to form, our algorithm injects too much CR energy because the estimated pressure jump is initially too large. While this causes an increased compressibility in comparison to the exact solution, the algorithm recovers as soon as the shock and post-shock regime have formed and then performs correctly.

We note that our second-order hydrodynamic scheme with a fixed mesh exhibits chequerboard instabilities within 
Table 1. Initial set-up of our shock tubes and resulting shock strengths.

\begin{tabular}{lcccccccr}
\hline \hline & $\rho_{5}^{(1)}$ & $\rho_{1}$ & $P_{5}$ & $P_{1}$ & $X_{\text {cr5 }}$ & $X_{\text {crl }}$ & $x_{\mathrm{s}}^{(2)}$ & \multicolumn{1}{c}{$\mathcal{M}_{1}$} \\
\hline th & 1 & 0.125 & 63.499 & 0.1 & 0 & 0 & 3.88 & 10.00 \\
th+inj & 1 & 0.125 & 63.499 & 0.1 & 0 & 0 & 4.74 & 9.56 \\
CR & 1 & 0.125 & 51.516 & 0.1 & 2 & 1 & 3.90 & 10.00 \\
CR+inj & 1 & 0.125 & 51.516 & 0.1 & 2 & 1 & 4.78 & 9.56 \\
\hline
\end{tabular}

(1) The left-half space in the initial conditions $(x<5)$ is denoted with a subscript 5 while the right-half space $(x>5)$ has a subscript 1 .

(2) The density jump at the shock is given by $x_{\mathrm{s}}=\rho_{2} / \rho_{1}$.

the rarefaction wave at the position of the high-pressure state of the initial conditions. These are known numerical artefacts that result from the large expansion velocity relative to the fixed mesh and cause the approximate Riemann finder to return erroneously the characteristics of the shock state instead of the intermediate state representing the rarefaction fan. ${ }^{6}$ If run with the moving mesh, these chequerboard instabilities vanish identically because the lower velocity relative to the moving mesh enables the Riemann solver to correctly identify the intermediate states.

\subsection{Sedov-Taylor blast wave}

We are interested in how CR shock acceleration changes the expansion behaviour of spherical shocks and whether it breaks self-similarity of the exact solution in the case without CR acceleration. To this end, we perform a sequence of simulations of the Sedov-Taylor problem, i.e. a point explosion leading to an energy-driven, spherically symmetric strong shock that expands into a cold external medium with negligible pressure. The curved geometry of the shock surface in this problem is well suited to explore the behaviour of our CR shock acceleration algorithm with a fully unstructured 3D Voronoi mesh free of any preferred directions in the initial conditions.

The initial Voronoi mesh is generated by distributing mesh-generating points randomly in the unit box $(x, y, z) \in$ $[0,1]^{3}$. We relax the mesh via Lloyd's algorithm (1982) to obtain a glass-like configuration. The initial conditions are as follows: we fill the box with a uniform density gas of $\rho_{0}=1$ and pressure $P_{0}=10^{-4}$, the initial velocities are identically zero and we adopt an thermal adiabatic index of $\gamma=5 / 3$. We inject an initial thermal energy of $E_{0}=1$ into the central mesh cell. To explore numerical convergence, we repeat the simulations at various resolutions corresponding to $25^{3}, 50^{3}$, and $100^{3}$ cells. In these simulations, we follow hydrodynamics without self-gravity and - in case of CR-shock acceleration - assume a large CR acceleration efficiency of $\zeta=0.5$ in order to examine our algorithm under extreme conditions. We do not account for non-adiabatic CR loss processes.

Figure 2 shows cross-sections through the centre of the $3 \mathrm{D}$ volume of our simulation with $\mathrm{CR}$ shock acceleration at $t=0.1$. We chose the low-resolution simulation with $50^{3}$ cells so that features of the CR distribution can be related to individual mesh cells, which are also shown in the images. The

6 We caution that a better reconstruction scheme for the fixedmesh (that avoids the pairwise decoupling) should also be able to correct for these inaccuracies. original glass-like distribution of mesh cells is still visible in the corners of the maps. We note that the cross-section of a 3D Voronoi mesh is in general no longer a Voronoi tessellation. We address the details of the CR shock acceleration algorithm in the density cross-section (Fig. 2, top left). The area in between the two white contour lines shows the shock zone, i.e. it is characterised by converging flows for which spurious shocks such as tangential discontinuities and contacts have been filtered out. The velocity convergence along the direction of shock propagation is maximised in the cell labelled as shock surface. The collection of shock-surface cells is enclosed by the black contour lines. As before in the case of the (strong) shock tube simulations, the shock zone extends typically over three to four cells along the shock normal and the inner boundary that connects to the post-shock region often coincides with the shock surface cells. This implies that we typically inject CRs into two (or rarely three) cells along the direction of shock propagation $\boldsymbol{d}_{\mathrm{s}}$ as inferred by the local orientation of the temperature gradient.

The shock velocity decreases steeply with time in the Sedov solution. Thus, most of the specific energy is dissipated at early times. Because we funnel a fixed fraction of the dissipated energy into CRs in our model, the CR energy per unit mass, $u_{\mathrm{cr}}$, decreases also steeply with radius (Fig. 2, top right). In contrast, the total pressure peaks at the shock surface and plateaus to a constant value inside the blast wave (Fig. 2, bottom left), indicating that the density decrease due to adiabatic expansion is exactly countered by the increase in specific energy. Most interestingly, the relative CR pressure, $X_{\mathrm{cr}}=P_{\mathrm{cr}} / P_{\mathrm{th}}$, increases steeply with increasing distance from the shock (Fig. 2, bottom right). This is because adiabatic expansion of a composite of $\mathrm{CR}$ and thermal gas favours the $\mathrm{CR}$ pressure over the thermal pressure due to the softer equation of state of CRs.

For our adopted value for the CR acceleration efficiency $\zeta=0.5$, we obtain a $\mathrm{CR}$ pressure ratio at the shock of $X_{\mathrm{cr} 0}=\left(\gamma_{\mathrm{cr}}-1\right) \varepsilon_{\mathrm{cr} 0} /\left[\left(\gamma_{\mathrm{th}}-1\right) \varepsilon_{\mathrm{th} 0}\right]=\zeta /[2(1-\zeta)]=0.5$. Adiabatic expansion over a density expansion factor $\delta$ yields

$$
X_{\mathrm{cr}}=\frac{P_{\mathrm{cr} 0} \delta_{\mathrm{cr}}^{\gamma_{\mathrm{c}}}}{P_{\mathrm{th} 0} \delta_{\mathrm{th}}}=X_{\mathrm{cr} 0} \delta^{-1 / 3} \approx 5\left(\frac{\delta}{10^{-3}}\right)^{-1 / 3},
$$

in agreement with the simulated average values. However, the cross-section of $X_{\text {cr }}$ shows a substantial scatter in this quantity at any given radius. A close inspection reveals radial 'fingers' of CR over-pressured pockets close to the shock surface. This can be traced back to the scatter in the temperature gradient that defines the opposite direction of shock propagation. This causes individual 'shock rays' that point opposite to the direction of shock propagation to sometimes converge in a single post-shock cell. As a result, we inject 

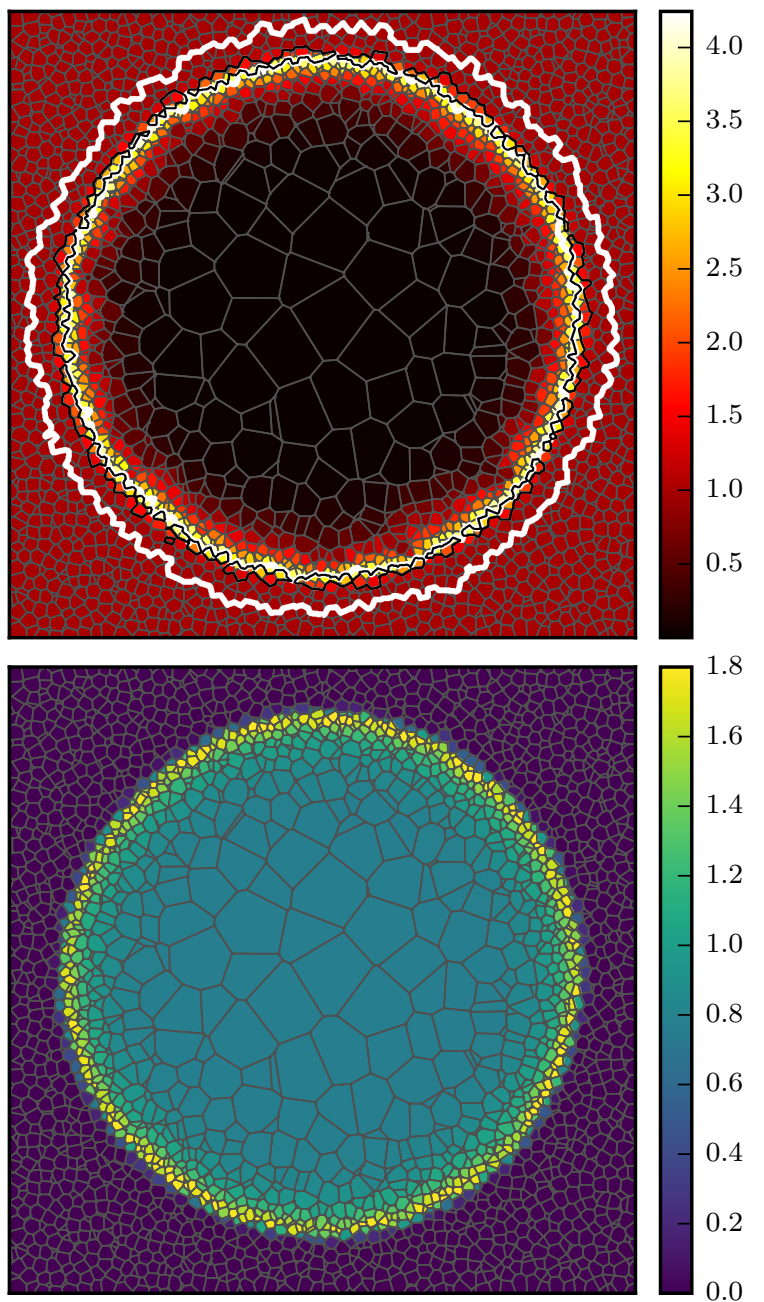
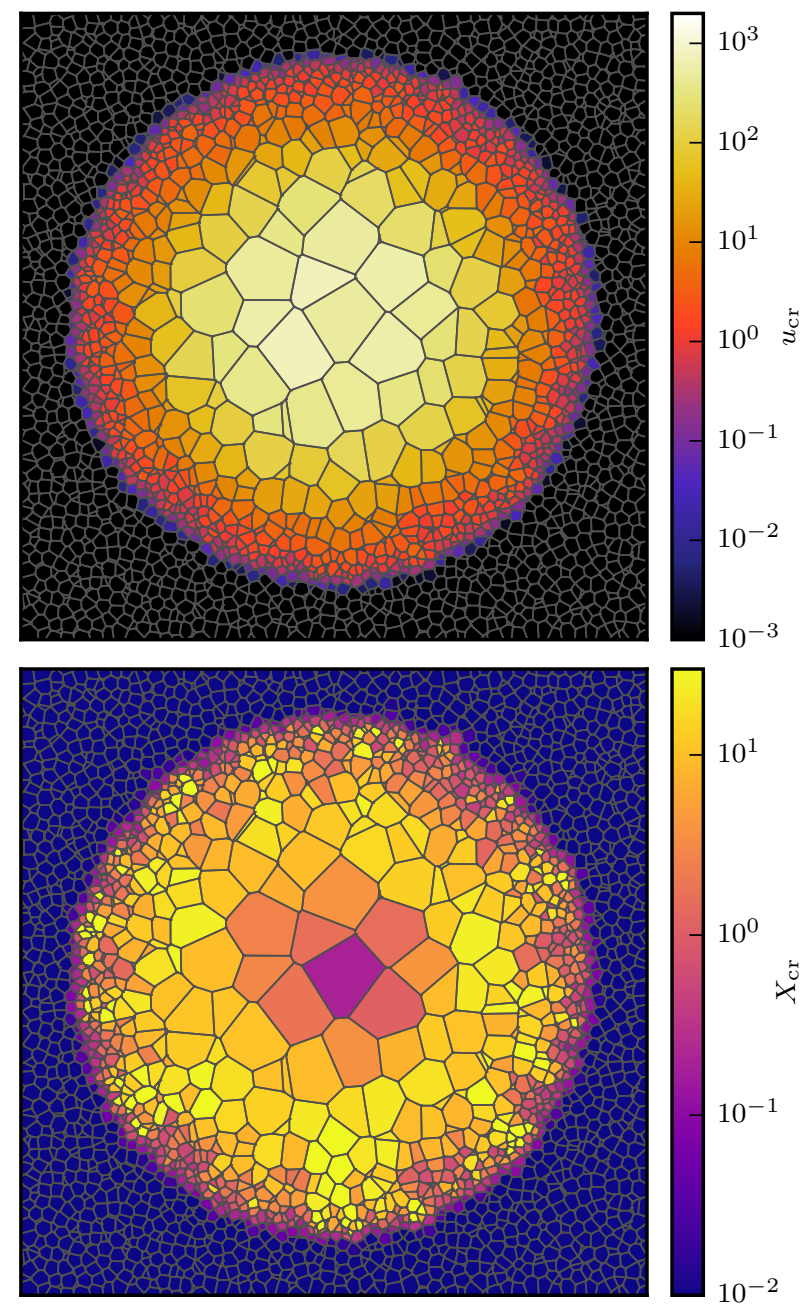

Figure 2. Sedov-Taylor blast wave with $\mathrm{CR}$ acceleration. We present cross-sections through the centre of the 3D simulation volume of our CR shock acceleration simulation with $50^{3}$ cells at time $t=0.1$. Shown are the mass density $\rho$ (top left), the CR energy per unit mass $u_{\text {cr }}$ (top right), the total pressure $P_{\text {tot }}=P_{\text {th }}+P_{\text {cr }}$ (bottom left), and the relative CR pressure $X_{\mathrm{cr}}=P_{\text {cr }} / P_{\text {th }}$ (bottom right). In the density cross-section, we also visualise the shock zone (bounded by two thick white contour lines) as well as the shock surface (thin black contour).

CR energy (and accordingly remove thermal energy) twice in those cells and consequently skip CR injection in adjacent cells if those are missed by a 'shock ray'. While this may represent a weakness of our CR injection algorithm, we emphasise that physical CR diffusion (which is neglected here for clarity) will smooth out these irregularities on short timescales. ${ }^{7}$

In order to quantify these visual impressions, we would like to compare radial profiles of azimuthally averaged quantities to exact solutions. Unfortunately, a solution of the Sedov problem for a two-component fluid which accounts for $\mathrm{CR}$ acceleration at the expanding shock does not exist. In order to check whether such a simulation with CR acceleration obeys a self-similar behaviour, we need to derive a constant adiabatic index that best describes the solution.

\footnotetext{
7 The fact that this scatter in $X_{\text {cr }}$ can be maintained by the code, is a manifestation of the low numerical diffusivity of our moving mesh technique.
}

To this end, we show the radial profile of the effective adiabatic index $\gamma_{\text {eff }}$ (equation 30) of our $100^{3}$-cell simulation at $t=0.1$ (Fig. 3, top left). It transitions from the thermal gas value of $\gamma=5 / 3$ outside the shock to a value that is almost fully CR dominated well inside the blast wave, because the adiabatic expansion favours the CR pressure over the thermal gas pressure (Fig. 3, bottom left). Overall, a value of $\gamma=7 / 5$ characterises the average behaviour of $\gamma_{\text {eff }}$ well, as can be validated by comparing the total simulated pressure profile to the exact profile of a polytropic fluid of $\gamma=7 / 5$.

In the case of a single polytropic fluid, the shock radius of a $3 \mathrm{D}$ explosion evolves as

$$
r_{\text {shock }}(t)=\left(\frac{E_{0}}{\alpha \rho_{0}}\right)^{1 / 5} t^{2 / 5},
$$

where $\alpha=(0.49,0.851)$ for $\gamma=(5 / 3,7 / 5)$ according to Sedov (1959). In Figure 3 (top right) we show the time evolution of the azimuthally averaged shock radius of our simulation without (blue points) and with CR acceleration (red 

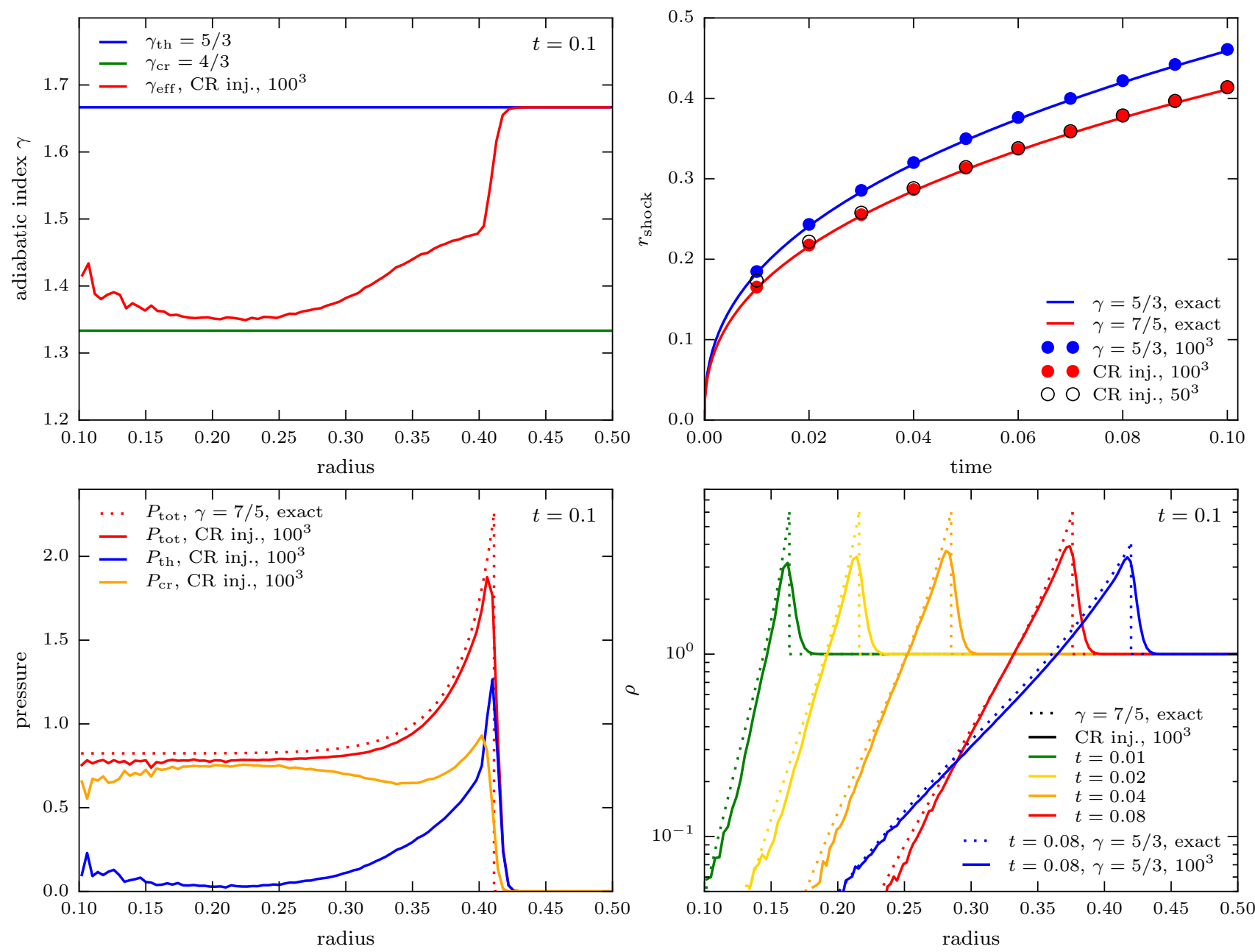

Figure 3. 3D Sedov-Taylor blast wave simulations with CR shock acceleration. All radial profiles show azimuthally averaged quantities and are centred on the centre-of-mass of the initial cell into which the energy was injected. Top left: we show the effective adiabatic index $\gamma_{\text {eff }}$ of our $100^{3}$-cell simulation, which transitions from the thermal gas value of $\gamma=5 / 3$ outside the shock to a value that is almost fully CR-dominated well inside the blast wave. Top right: time evolution of the azimuthally averaged shock radius of our simulations (data points, determined from the shock surface property) and the exact solution (solid line). Except for the innermost data point at $t=0.01$, the $100^{3}$-cell simulation has well converged to the $50^{3}$-cell run. Bottom left: we compare the simulated total pressure (solid red) to the exact solution for a polytropic gas with $\gamma=7 / 5$ (dotted red) and show the contributions from the partial pressures of the thermal (blue) and CR constituents (orange). Bottom right: time sequence of the density profiles of our simulation with CR acceleration (solid, green-to-red) and the exact solution for a polytropic gas with $\gamma=7 / 5$ (dotted, green-to-red). Also shown is the density profile of a simulation without $\mathrm{CR}$ acceleration (solid blue) and the exact solution (dotted blue), which has advanced further in comparison to the case with $\mathrm{CR}$ acceleration.

points). In agreement with our shock tube simulations, the blast wave that accelerates CRs propagates at a slower rate in comparison to the case without CR acceleration. This comes about because of the softer effective equation of state of the composite fluid that allows higher post-shock densities and thus cannot advance as fast. We demonstrate that our $100^{3}$-cell simulation has converged to the $50^{3}$-cell run, with the exception of the innermost data point at $t=0.01$. These measured shock positions compare well to the exact values for $\gamma=5 / 3$ and $7 / 5$, respectively. This shows that a constant value for $\gamma_{\text {eff }}$ captures the overall expansion behaviour of the blast wave despite the fact that the adiabatic index is changing as a function of radius. This can be explained because in our model, a fixed fraction of the dissipated energy is in- jected into CRs so that the CR pressure ratio experiences the same adiabatic expansion for the same dilution factor.

This can be substantiated further by comparing the density profiles of our simulation with CR acceleration (solid, green-to-red) to the self-similar analytical solution for a polytropic gas with $\gamma=7 / 5$ (dotted, green-to-red) at different times. The shapes of the density profiles stay approximately self-similar and show similar deviations from the analytical solution as the density profile of a simulation without CR acceleration (solid blue) and the exact solution (dotted blue). However, the CR acceleration run differs by a larger factor from the exact solution at the shock in comparison to the polytropic run with $\gamma=5 / 3$. This is because our approximation of $\gamma=7 / 5$ clearly breaks down at the shock, where we obtain an effective adiabatic index, $\gamma_{\text {eff }}$, and shock 
compression ratio, $x_{\mathrm{s}}$, of

$$
\begin{aligned}
\gamma_{\mathrm{eff}} & =\frac{\gamma_{\mathrm{cr}} X_{\mathrm{cr} 0}+\gamma_{\mathrm{th}}}{X_{\mathrm{cr} 0}+1}=\frac{14}{9}=1 . \overline{5}, \text { and } \\
x_{\mathrm{s}} & =\frac{\gamma_{\mathrm{eff}}+1}{\gamma_{\mathrm{eff}}-1}=\frac{23}{5}=4.6 .
\end{aligned}
$$

The expression for $x_{\mathrm{s}}$ in equation (63) is valid in the limit of a strong shock, and the numerical values are obtained by adopting the assumed CR acceleration efficiency of $\zeta=0.5$. We conclude that the azimuthally averaged post-shock density falls short of the theoretically expected value by about $15 \%$, which is a similar deficiency factor as for the polytropic run with $\gamma=5 / 3$.

\subsection{Isolated models of galaxy formation}

We now assess the impact of CR pressure feedback on the formation and evolution of isolated disk galaxies in dark matter haloes that range in mass from $10^{10}$ to $10^{12} \mathrm{M}_{\odot}$. We would like to understand how exactly CRs accelerated by supernova remnants are able to regulate star formation by means of their pressure feedback. Moreover, we are interested how the structure of the ISM changes as a consequence of CR feedback and whether this has any direct consequences on magnetic dynamo amplification mechanisms. ${ }^{8}$ We are in particular interested how the strength of CR pressure feedback depends on halo mass, because the global star conversion efficiency needs to be a strong function of halo mass in order to explain the shallow faint end of the galaxy luminosity function. While strong galactic outflows in form of winds are potentially responsible for the majority of this mass-dependent regulation of star formation, we will study here how much of this reduction of star formation is directly related to the additional non-thermal pressure support of CRs.

We model the ISM by an effective pressurised equation of state and follow radiative cooling and star formation using the approach by Springel \& Hernquist (2003). We employ ideal MHD using cell-centred magnetic fields and the Powell et al. (1999) scheme for divergence control (Pakmor et al. 2011; Pakmor \& Springel 2013). In these simulations, we account for CR injection at supernovae with a CR energy injection efficiency of $\zeta_{\mathrm{SN}}=0.1$, follow advective $\mathrm{CR}$ transport, and account for adiabatic changes of the $\mathrm{CR}$ energy as well as Coulomb and hadronic CR cooling as detailed in Sects. 3.2 and 3.3. ${ }^{9}$ Note that in these simulations, we use the sub-resolution model of CR injection at supernovae (Sect. 3.2) and do not employ our explicit shock finding method and associated CR acceleration. Also, we neglect active CR transport in the form of anisotropic diffusion and streaming, which is studied in detail in a companion paper (Pakmor et al. 2016c) and only consider advective CR transport with the gas. Because in this approximation, CRs are tied to the gas, they cannot diffuse ahead of the gas and, as a result of this, their pressure gradient cannot impulsively

${ }^{8}$ Here, we report values for the magnetic field strength in cgs units.

9 Here and in Sect. 4.4, we use the collisional heating rate due to Coulomb interactions only, where $\Gamma_{\text {th }}=-\Lambda_{\text {Coul }}=\tilde{\lambda}_{\text {th }} n_{\mathrm{e}} \varepsilon_{\mathrm{cr}}$ and $\tilde{\lambda}_{\text {th }}=2.78 \times 10^{-16} \mathrm{~cm}^{3} \mathrm{~s}^{-1}$. start to dominate the force balance, which is a necessary condition for accelerating the gas in order to launch a powerful galactic wind.

The initial conditions are given by a prescribed dark matter potential that results from a density distribution motivated from cosmological simulations (Navarro et al. 1997). The density profile is characterised by a concentration parameter, which we keep fixed at a value of $c_{200}=12$ across our halo mass range. Hence, the haloes are scaled versions of each other which would evolve in a self-similar way if we only considered gravity and ideal (magneto-)hydrodynamics. However, the physics of cooling, star formation and CRs breaks this scale-invariance and any quantitative differences among the haloes can be directly related to additional scales introduced by these processes.

We adopt a hydrostatic gas distribution that is initially in equilibrium within the halo. We assume that the halo carries a small amount of angular momentum, parametrized by a spin parameter $\lambda=0.05$, which is close to the median found in cosmological simulations. In all cases, we use a baryon mass fraction of $\Omega_{\mathrm{b}} / \Omega_{\mathrm{m}}=0.155$. The magnetic field is initialised through a uniform homogeneous seed field along the $x$-axis with an initial strength of $10^{-10} \mathrm{G}$, and there are no CRs in the initial setup.

In the initial conditions, we have $10^{6}$ gas cells inside the virial radius, each of which with a target mass of $1.55 \times$ $10^{3} \mathrm{M}_{\odot} \times M_{10}$, where $M_{10}=M_{200} /\left(10^{10} \mathrm{M}_{\odot}\right)$. This target gas mass also corresponds to the typical mass of a stellar macroparticle representing a stellar population. We enforce that the mass of all cells is within a factor of two of the target mass by explicitly refining and de-refining the mesh cells. We additionally require that adjacent cells differ in volume by less than a factor of 10 and refine the larger cell if this condition is violated.

When we evolve one of these haloes forward in time, radiative cooling diminishes the pressure support of the gas in the centre, which then collapses while conserving its specific angular momentum, thus settling into a rotationally supported cold disk. In the disk, the gas is compressed by self-gravity to sufficiently high densities that star formation ensues. In our model, CRs are injected into the ambient ISM surrounding stellar macro-particles, providing the gas with additional non-thermal pressure. Because the energy stored in CRs is subject to different dissipative loss processes in comparison to the thermal gas, their additional pressure support alters the radiative cooling of galaxies. This reduces the overall cooling efficiency of gas in haloes, directly resulting in a reduction of the condensated phase of cold gas and quenches subsequent star formation (see Fig. 4, left-hand panel). In agreement with findings by Jubelgas et al. (2008), during the first 2 Gyr of the simulation this suppression due to CR pressure feedback is larger for more shallow gravitational potential wells, which are provided by the stars and the dark matter halo, and hence the smaller galaxies are more strongly quenched. However, this trend is reversed at late times because the galaxy in the model without CRs has exhausted its available gas reservoir.

The star formation rate peaks around $0.1-0.2 \mathrm{Gyr}$ and then declines exponentially, so that most of the stars are formed in the first Gyr. As the first stars are forming, the $\mathrm{CR}$ energy density in the disk quickly reaches equilibrium with the thermal energy density and dominates the internal 

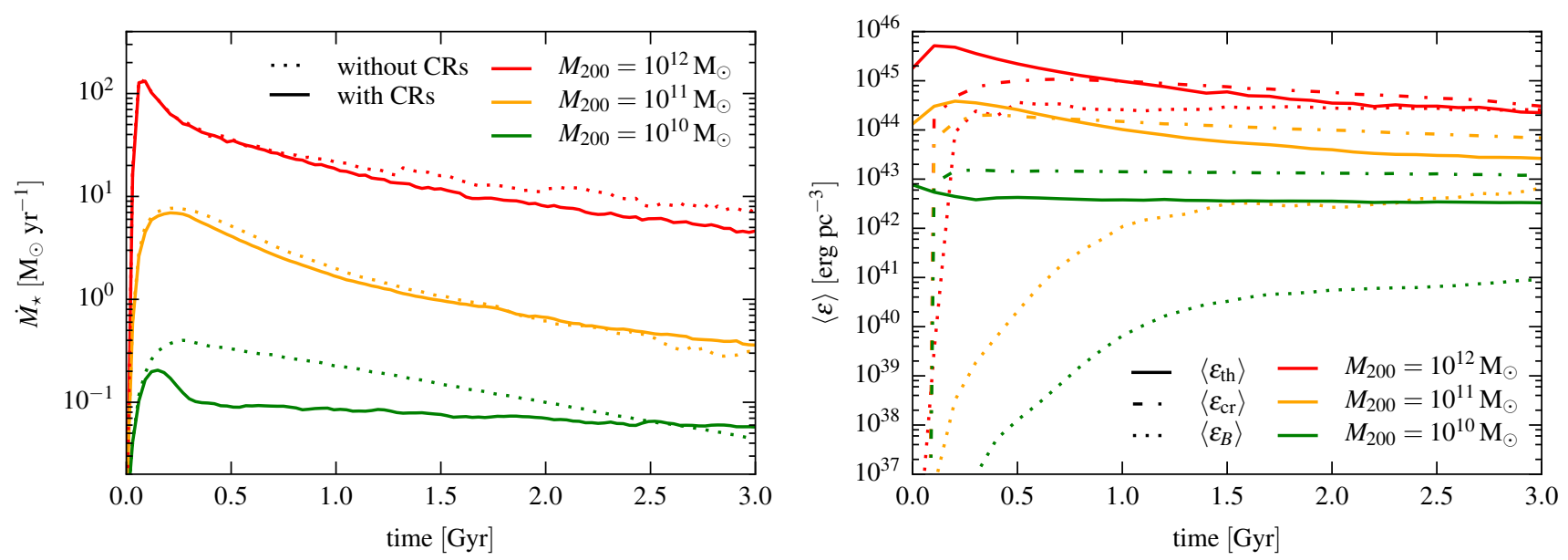

Figure 4. Time evolution of the star formation rate (left-hand panel) and the average energy densities in a disk of radius 10 kpc and height $1 \mathrm{kpc}$ that is centred on the mid-plane (right-hand panel). Different halo masses with $10^{10}$, $10^{11}$, and $10^{10} \mathrm{M}_{\odot}$ are colour coded. Simulations with advective CR feedback (solid lines) suppress star formation more strongly in smaller galaxies in comparison to simulations without CRs (dotted lines). The right panel shows the evolution of the thermal energy density, CR energy density, and magnetic energy density, respectively, in our MHD simulations with CR feedback.

energy budget soon thereafter (see Figure 4, right panel). Smaller galaxies show a shorter time scale for energy equilibration and the CR energy dominance, $\left\langle\varepsilon_{\mathrm{cr}}\right\rangle /\left\langle\varepsilon_{\mathrm{th}}\right\rangle$, is also larger in small galaxies because of the shallower potential wells which amplify the impact of CR pressure feedback.

Figure 5 shows the disk at time $t=1.5 \mathrm{Gyr}$ after the start of the evolution and compares the simulations with and without advective CR transport (top and bottom panels, respectively). At this time, the gas density strongly peaks in the centre of the disk, where most of the stars are formed and thus most of the CRs are injected there, which is reflected in the distribution of CR energy density. A visual difference of the density distributions between the two types of simulations is apparent. While the simulation without CR feedback shows the smoothed density and thermal energy of the ISM as predicted by the subgrid-scale model of the pressurised effective equation of state, the ISM is very clumpy in the run with CR feedback. Every low-density cavity corresponds to the location of a star forming region (represented by a stellar macro-particle) and was evacuated by the Sedov-Taylor blast wave that has formed as a result of the CR energy deposition of the collection of supernovae with an energy that corresponds to the stellar mass formed (Sect. 3.2). This becomes evident from the tight spatial correlation of the density cavities and peaks in the CR energy density (top left and middle panel of Fig. 5). The cavity sizes grow larger with galacto-centric radius because of the lower ambient gas densities that the blast waves encounter there. We note that the cavity morphologies are not smoothed due to numerical effects thanks to the very low numerical diffusivity of the moving mesh technique that enables mesh cells to co-rotate with the rotating disk. Eventually the cavities are sheared and dispersed by differential rotation on time scales that increase with galacto-centric radius, leaving the supernovaeblown cavities at galactic outskirts intact for longer times.

While CR streaming and diffusion are believed to smooth out the inhomogeneous CR distribution to some extent, we envision that some of the results here should also carry over to situations where more realistic spatially varying diffusion coefficients are employed. Upstream of supernova remnant shocks, high-energy CRs drive nonresonant hybrid plasma instabilities (Bell 2004), which generate strong electromagnetic turbulence that causes the CRs to experience on average one scattering event per gyro revolution. The resulting motion of CRs can be described by a diffusive transport that is characterised by a Bohm diffusion coefficient of $\kappa_{\mathrm{B}} \approx p \beta m_{\mathrm{p}} c^{2} /(3 e B) \approx 3 \times$ $10^{21}\left(p m_{\mathrm{p}} c^{2} / \mathrm{GeV}\right)(B / \mu \mathrm{G})^{-1} \mathrm{~cm}^{2} \mathrm{~s}^{-1}$ (Stage et al. 2006). If the CRs propagate far into the upstream of the shock or if the supernova remnant shock wave has sufficiently slowed down and entered the snowplough phase so that the CRs that have been trapped in the interior of the remnant can escape the supernova environment, the CR-wave scattering rate starts to decline. As a result, the CR mean free path increases so that the effective diffusion coefficient - as long as the mode of CR propagation can reliably be described by a diffusion process - approaches the average galactic value of $3 \times 10^{28} \mathrm{~cm}^{2} \mathrm{~s}^{-1}$.

In addition to the increased clumpiness of the ISM, the CR feedback simulation shows a larger density scale height in comparison to the MHD simulation without CRs (lefthand panels of Fig. 5). This increase is due to the additional $\mathrm{CR}$ pressure that is subject to different dissipative loss processes in comparison to the thermal gas, and has a cooling time well above the radiative loss time of thermal gas. In Fig. 6, we compare the gas density and CR energy density after 1.5 Gyr in our CR simulations for different halo masses of $10^{10}, 10^{11}$, and $10^{12} \mathrm{M}_{\odot}$. The aspect ratio of the gas disk (disk length-to-height) decreases for smaller disks to almost unity for the $10^{10} \mathrm{M}_{\odot}$ halo as a result of the relatively larger impact of CR pressure feedback in smaller haloes. This is in qualitative agreement with the results of smoothed particle hydrodynamics simulations by Jubelgas et al. (2008), although the extents of the disks in our moving mesh simulations are nearly twice of that of their simulations owing to improvements of the hydrodynamical method here. 

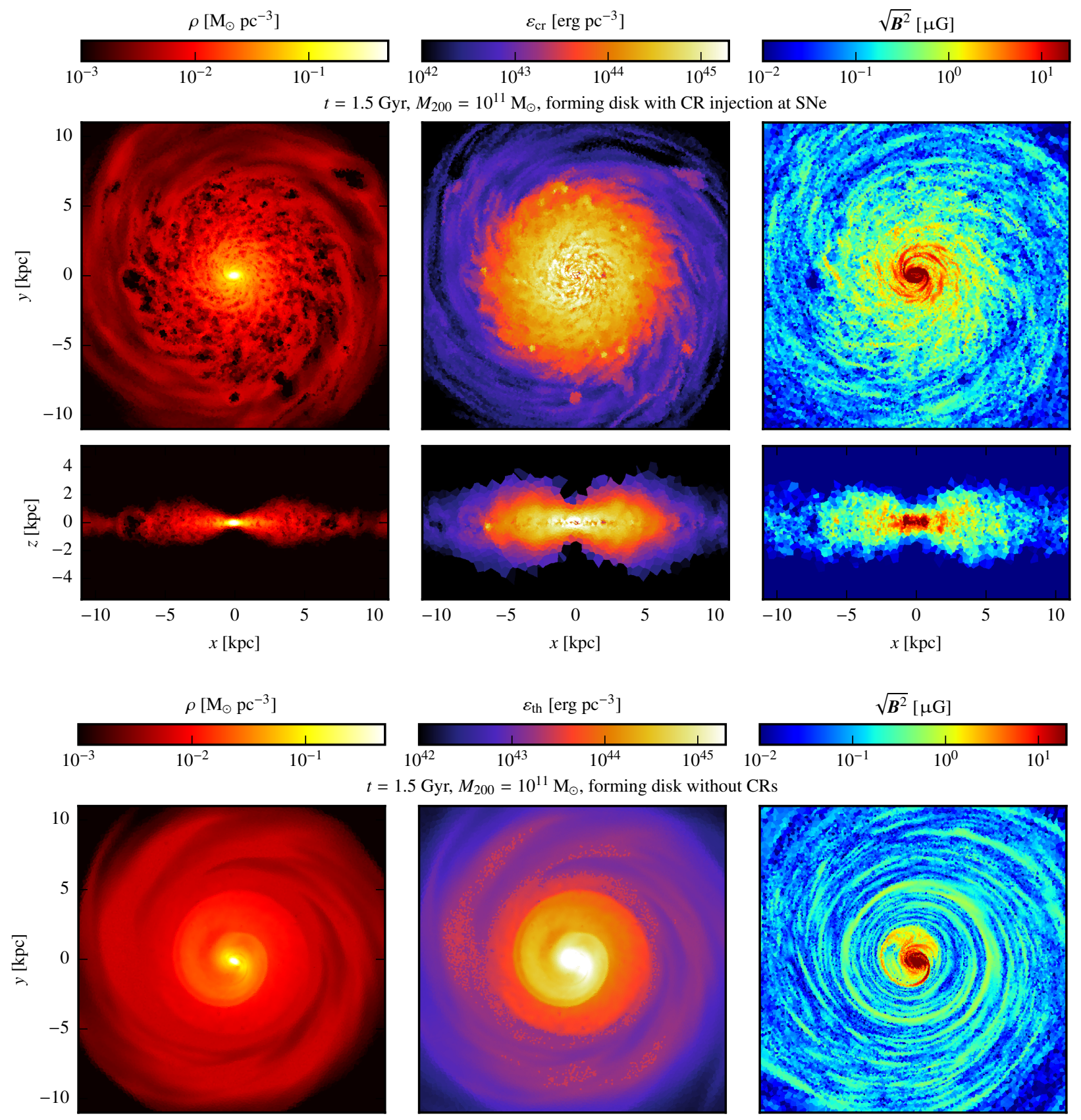

$t=1.5 \mathrm{Gyr}, M_{200}=10^{11} \mathrm{M}_{\odot}$, forming disk without CRs
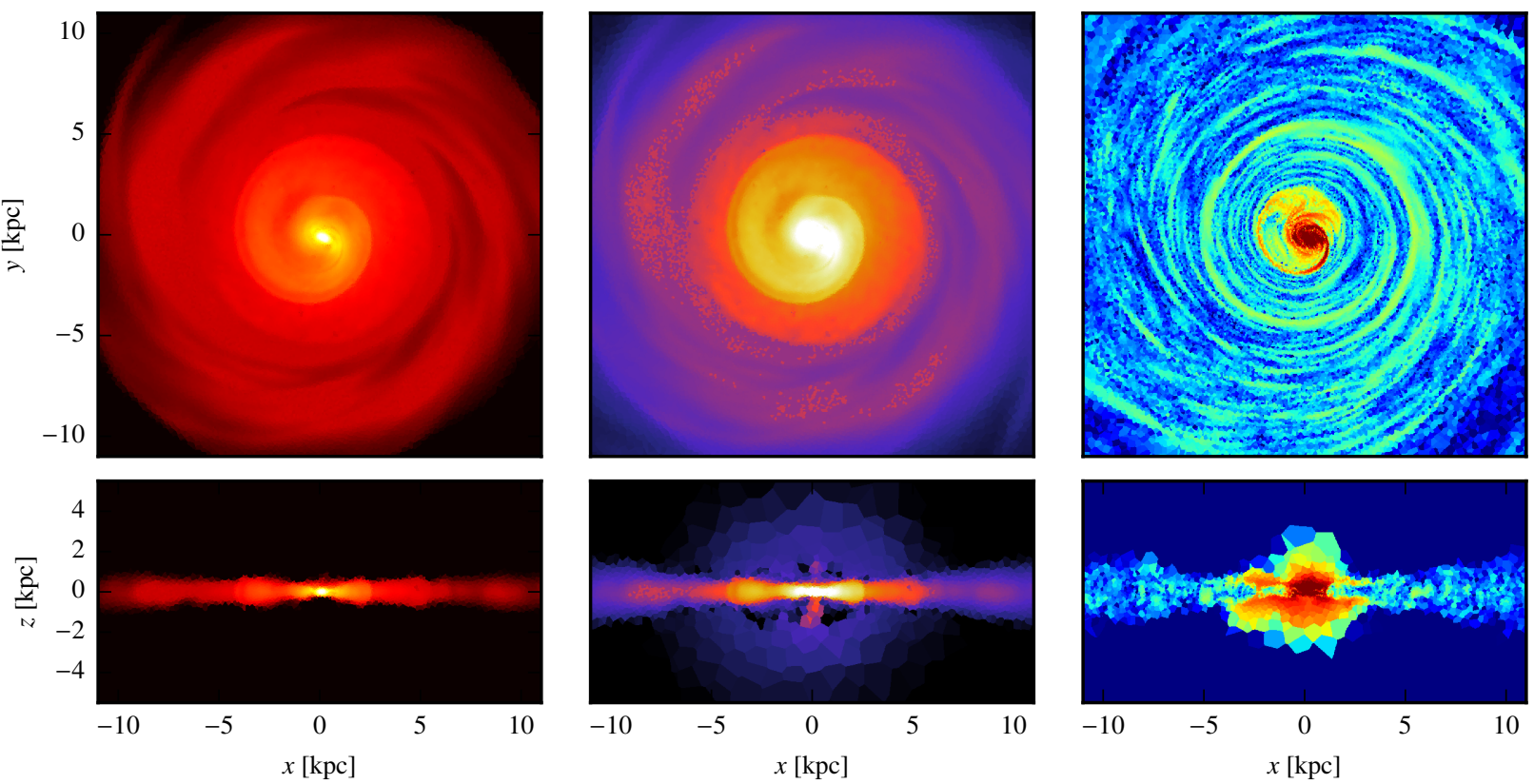

Figure 5. Properties of the gas disk in our $10^{11} \mathrm{M}_{\odot}$ halo after 1.5 Gyr in MHD simulations where we inject CRs with our supernova remnant model and follow their advection with the gas (top six panels) and simulations without CRs (bottom six panels). We show cross-sections of gas properties in the mid-plane of the disk (face-on views) and vertical cut-planes through the centre (edge-on views) of the gas density (left-hand panels), CR and thermal energy density (middle panels, top and bottom, respectively), and the magnetic field strength (right-hand panels). 

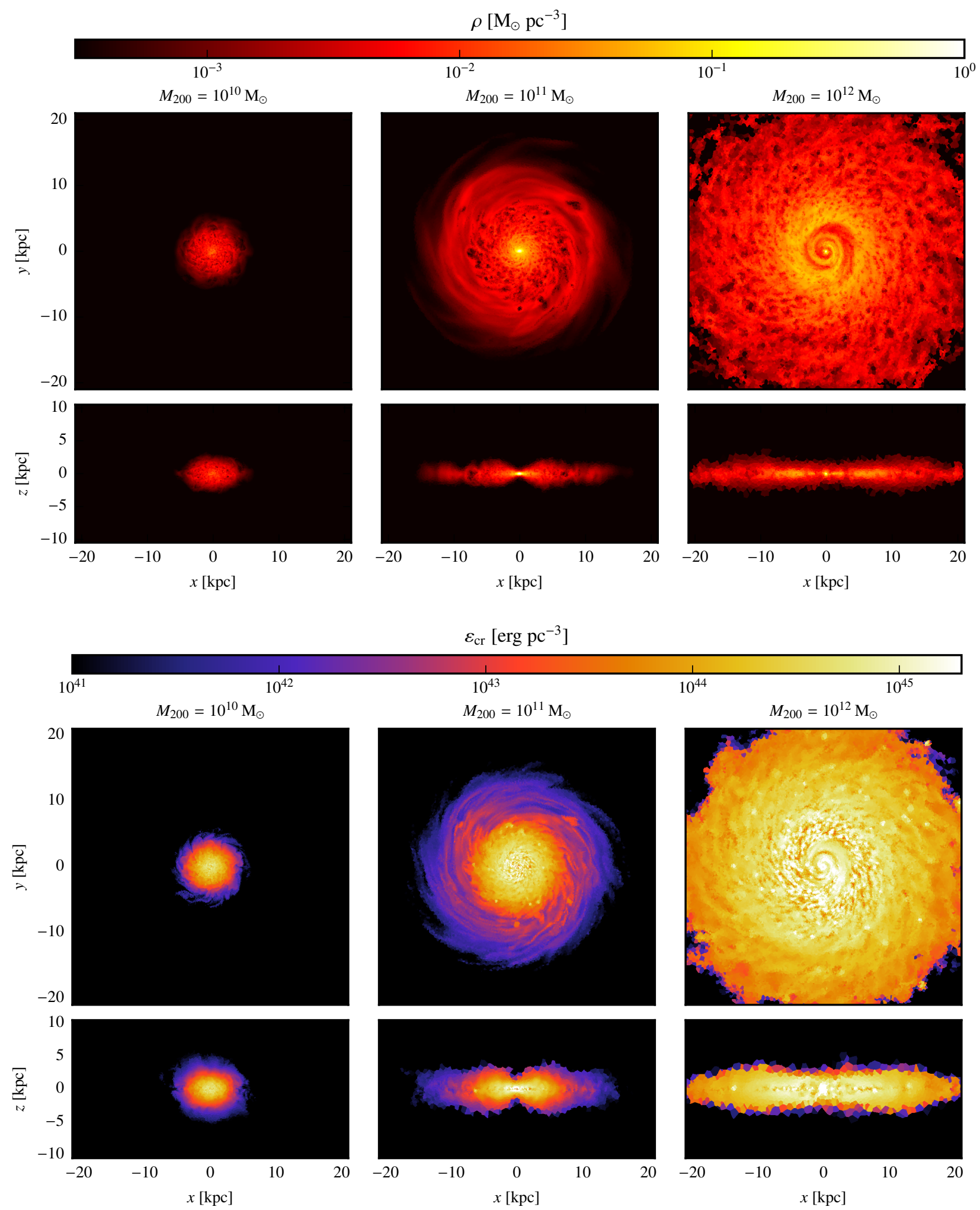

Figure 6. Properties of the gas disk after 1.5 Gyr in MHD simulations where we inject CRs with our supernova remnant model and follow their advection with the gas. We present the gas density (top six panels) and CR energy density (bottom six panels), and show cross-sections in the mid-plane of the disk (face-on views) and vertical cut-planes through the centre (edge-on views). From the left to right, we compare different halo masses of $10^{10}, 10^{11}$, and $10^{12} \mathrm{M}_{\odot}$. 

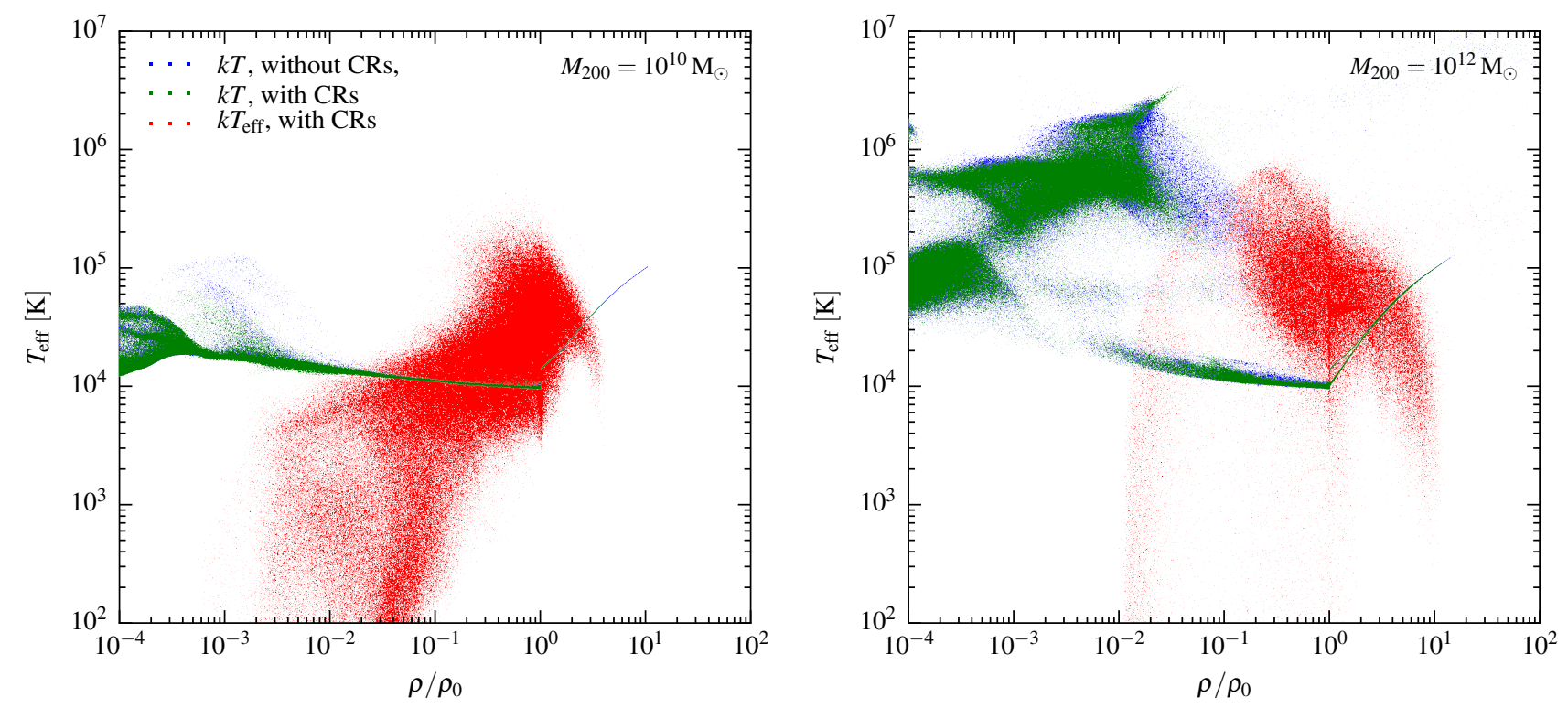

Figure 7. Distribution of the gas in the temperature-density plane for our halo of mass $10^{10} \mathrm{M}_{\odot}$ (left-hand panel) and $10^{12} \mathrm{M}_{\odot}$ (right-hand panel). All densities are scaled to the star formation threshold $\rho_{0}=4.05 \times 10^{-25} \mathrm{~g} \mathrm{~cm}^{-3}$. We compare the thermal gas properties $(T$ and $\rho$ ) in our simulation without CR physics (blue points) to the simulation with advective CR physics (green points). To assess the impact of CR pressure feedback, we also show the CR pseudo temperature $T_{\mathrm{eff}}=P_{\mathrm{cr}} \mu_{\mathrm{mw}} m_{\mathrm{p}} \rho^{-1}$ versus gas density (red). We show the systems at time $t=2.0 \mathrm{Gyr}$ after the start of the evolution.

Comparing the distribution of CR energy density across the mass sequence reveals a more homogeneous distribution in the larger halo of mass $10^{12} \mathrm{M}_{\odot}$ : star formation proceeds here throughout the entire disk rather than being only concentrated towards the centre as in the smaller haloes, and hence most of the CRs are injected more homogeneously into the ISM.

In the initial phase of the galaxy assembly (within the first $200 \mathrm{Myr}$ ), the magnetic field strength is exponentially amplified on small length and time scales. This is consistent with a turbulent small-scale dynamo that operates to an average field strength of about $10 \mu \mathrm{G}$ for our Milky Way-type halo of $10^{12} \mathrm{M}_{\odot}$ and to $10^{-2} \mu \mathrm{G}$ for our less massive galaxies (see right panel in Fig. 4). After this initial assembly phase the disk has finished forming and the dominating differential rotation of the gas in the disk stretches the magnetic flux tubes so that the coherence scale can grow. In the Milky Way-type galaxy, the field strength has saturated and only grows in scale while the magnetic field continues to grow exponentially in the smaller haloes, but on much longer time scales, suggesting that the dominant dynamo amplification mechanism has changed. Interestingly, the morphology of the magnetic field is also substantially modified by CR feedback (right panels of Fig. 5). While the field structure is very regular in the case without CRs, it attains a chaotic small-scale structure which is superimposed on the dominant azimuthal component. Apparently, the additional turbulent velocity field as a result of the supernova explosions has intensified the dynamo action which further amplified the field.

We complement our discussion on the exact mechanisms of $\mathrm{CR}$ pressure feedback in regulating star formation by considering the distribution of the gas in the temperaturedensity plane for our small and massive galaxy (Fig. 7). We compare the thermal gas properties $(T$ and $\rho$ ) in our simulation without CR physics (blue points) to the simulation with $\mathrm{CR}$ pressure feedback (green points). As the cooling gas is infalling onto the disk, it is accelerated by the gravitational potential and shocks to the virial temperature of the halo. It continues to loose energy through radiative cooling and slowly moves onto the nearly isothermal branch of the ISM along which it moves to higher densities as it cools further up to the critical density of the star formation threshold. At this point, the ISM is parametrized by a stiff effective equation of state, which interpolates between the hot and cold phases and provides an effective pressure to the ISM.

Clearly, there are regions in the $T$ - $\rho$ plane, which are avoided by the thermal gas in the run with CR injection (visible by the dominant blue colour). This is due to CR pressure feedback as can be directly assessed by considering the CR pseudo temperature $T_{\text {eff }}=P_{\text {cr }} \mu_{\mathrm{mw}} m_{\mathrm{p}} \rho^{-1}$ (red points), which dominates over the thermal temperature at the density of interest, i.e., where the thermal gas has been pushed out in comparison to the simulation without CRs. We note that the normalisation of the CR pseudo temperature depends on the hadronic and Coulomb cooling rate of CRs. A temporarily increased cooling rate as a result of fresh CR injection would have lowered the CR pseudo temperature in comparison to our approach that is based on a CR equilibrium distribution (see Section 3.3.4). This explains differences to the MilkyWay type galaxy simulations by Jubelgas et al. (2008) that follow a simplified CR spectrum with a single power-law spectrum and momentum cutoff, which provides a temporal resolution of the Coulomb cooling rate. As a result, they find 
the CR pressure to be insufficient to significantly affect massive galaxies. ${ }^{10}$ This demonstrates the need of future work to model the spectral CR distribution in space and time to accurately follow Coulomb cooling processes.

\subsection{Cosmological simulations}

In order to study the impact of diffusive shock acceleration on cosmological structure formation shocks as well as to examine the generalised shock finder in combination with the $\mathrm{CR}$ shock acceleration algorithm in a non-trivial realisation of shock morphologies, we perform here non-radiative cosmological MHD simulations that do not include radiative cooling or star formation. We focus on analysing the spatial distribution of the $\mathrm{CR}$ distribution in relation to the cosmological structure formation shocks, and on Mach number statistics.

We adopt a standard cold dark matter model with a cosmological constant $(\Lambda \mathrm{CDM})$, as recently inferred by the Planck Collaboration et al. (2016). The cosmological parameters of our model are as follows: $\Omega_{\mathrm{m}}=\Omega_{\mathrm{dm}}+\Omega_{\mathrm{b}}=$ $0.3089, \Omega_{\mathrm{b}}=0.0864, \Omega_{\Lambda}=0.6911, h=0.6774, n_{\mathrm{s}}=0.9667$, and $\sigma_{8}=0.8159$. Here, $\Omega_{\mathrm{m}}$ denotes the total matter density in units of the critical density for geometrical closure, $\rho_{\text {crit }}=3 H_{0}^{2} /(8 \pi G) . \Omega_{\mathrm{b}}$ and $\Omega_{\Lambda}$ specify the density of baryons and the cosmological constant at the present day, respectively. The present day Hubble constant is parametrized as $H_{0}=100 h \mathrm{~km} \mathrm{~s}^{-1} \mathrm{Mpc}^{-1}, n_{\mathrm{s}}$ denotes the spectral index of the primordial power-spectrum, and $\sigma_{8}$ is the rms linear mass fluctuation within a sphere of radius $8 h^{-1} \mathrm{Mpc}$ extrapolated to $z=0$.

Initially, our simulation employed $2 \times 512^{3}$ gas and dark matter resolution elements, which were regularly distributed within a periodic box of comoving size $100 h^{-1} \mathrm{Mpc}$. As a result, the dark matter particles have masses of $4.6 \times 10^{8} h^{-1} \mathrm{M}_{\odot}$ and the gas cells have a target mass of $1.79 \times 10^{8} h^{-1} \mathrm{M}_{\odot}$. Using explicit refinement and de-refinement we ensure that the mass of all cells remains within a factor of two of the target mass. The gravitational force softening was of a spline form with a Plummer-equivalent softening length of $6.5 h^{-1} \mathrm{kpc}$ comoving.

Initial conditions were created by perturbing the homogeneous particle distribution with a realisation of a Gaussian random field characterised by the $\Lambda$ CDM linear power spectrum. The displacement field in Fourier space was laid down using the Zel'dovich approximation, and the amplitude of each random phase mode was drawn from a Rayleigh distribution. For the initial redshift we chose $z_{\text {init }}=127$ which translates to an initial temperature of the gas of $T_{\text {init }}=244.8 \mathrm{~K}$. The adiabatic index of the gas is set to $\gamma_{\text {th }}=5 / 3$.

In these simulations, we account for CR acceleration at resolved cosmological structure formation shocks and adopt

10 However, in order not to overestimate the CR cooling by artificially lowering the energy-weighted momentum during a supernova injection event, the formalism by Jubelgas et al. (2008) only injects CRs above a specific momentum, which guarantees that the spectral component can only grow. This implies a lower effective injection efficiency in comparison to a case that follows a multi-component CR distribution. a realistic value for the acceleration efficiency of $\zeta=0.1$ at all shocks exceeding a pre-shock Mach number of $\mathcal{M}_{1}=3$, and zero otherwise. For simplicity, we assume that the acceleration efficiency does not depend on magnetic shock obliquity. We adopt an ultra-relativistic CR population which translates to a CR adiabatic index of $\gamma_{\mathrm{cr}}=4 / 3$. As a result, the $\mathrm{CR}$ pressure ratio in the immediate post-shock regime amounts to $X_{\text {cr }}=\zeta /[2(1-\zeta)]=0.0 \overline{5}$, assuming a cold preshock gas with negligible pressure support characteristic of external accretion shocks. Once injected, we follow advective CR transport, account for adiabatic changes of the CR energy as well as Coulomb and hadronic CR cooling. The magnetic field is initialised as a uniform homogeneous seed field along the $x$-axis with an initial comoving strength of $10^{-15} \mathrm{G}$. There are no CRs in the initial setup.

Figure 8 shows a visualisation of the simulation volume at $z=0$ that reveals several quantities of relevance for $\mathrm{CR}$ acceleration at structure formation shocks as well as the successive CR transport. The lower panels show strong external shocks with Mach numbers exceeding $\mathcal{M}_{1} \sim 100$ that occur when the cosmic fluid accretes into cosmic filaments and super-cluster regions. Interior to these structures, for the most part weak shocks are visible. Still, most of the energy is dissipated inside collapsed structures due to the higher pre-shock density and the increased shock velocities there. In contrast, little energy is dissipated in cosmic voids. These results are in excellent agreement with previous work that uses non-radiative physics (Ryu et al. 2003; Pfrommer et al. 2006, 2007; Skillman et al. 2008; Vazza et al. 2009, 2011; Hong et al. 2014; Schaal \& Springel 2015), while the addition of radiative physics introduces new populations of shocks as a result of non-gravitational energy release (Pfrommer et al. 2007, 2008; Kang et al. 2007; Planelles \& Quilis 2013; Schaal et al. 2016).

The thermal pressure distribution traces the cosmic web as revealed by the overdensity map (Fig. 8, top panels). However, the pressure shows an increased contrast in comparison to the density distribution because of the multiplication with the temperature field that drops steeply outside the location of cosmological formation shocks. At collisionless shocks, not all the free energy is thermalized but a fraction is funnelled to CRs, provided the shock strength exceeds a critical Mach number. Hence the CR pressure represents a biased distribution of the thermal pressure distribution, with groups and super-cluster regions being prominently visible (Fig. 8, middle panels). While this compares qualitatively well with previous work (Pfrommer et al. 2008; Vazza et al. 2012), we defer a detailed comparison study to future work. The relative CR pressure traces the overall morphology of the cosmic web well, and attains values that typically range up to $X_{\mathrm{cr}}=0.0 \overline{5}$. As in the case of the Sedov explosion problem, individual mesh cells can have CR pressure ratios that scatter above this value if the 'shock rays' delineating the direction of shock propagation converge in post-shock cells. The low-density web in cosmic voids remains almost invisible and exhibits CR pressure ratios less than $10^{-4}$. This is because the shocks responsible for forming these structures are weak and dissipate little energy during their assembly.

A close inspection of the $X_{\text {cr }}$ projection reveals that this quantity does not peak toward the densest centres, but at the location of the strong external formation shocks, which are the primary sources of $\mathrm{CR}$ acceleration in cosmic structures. 

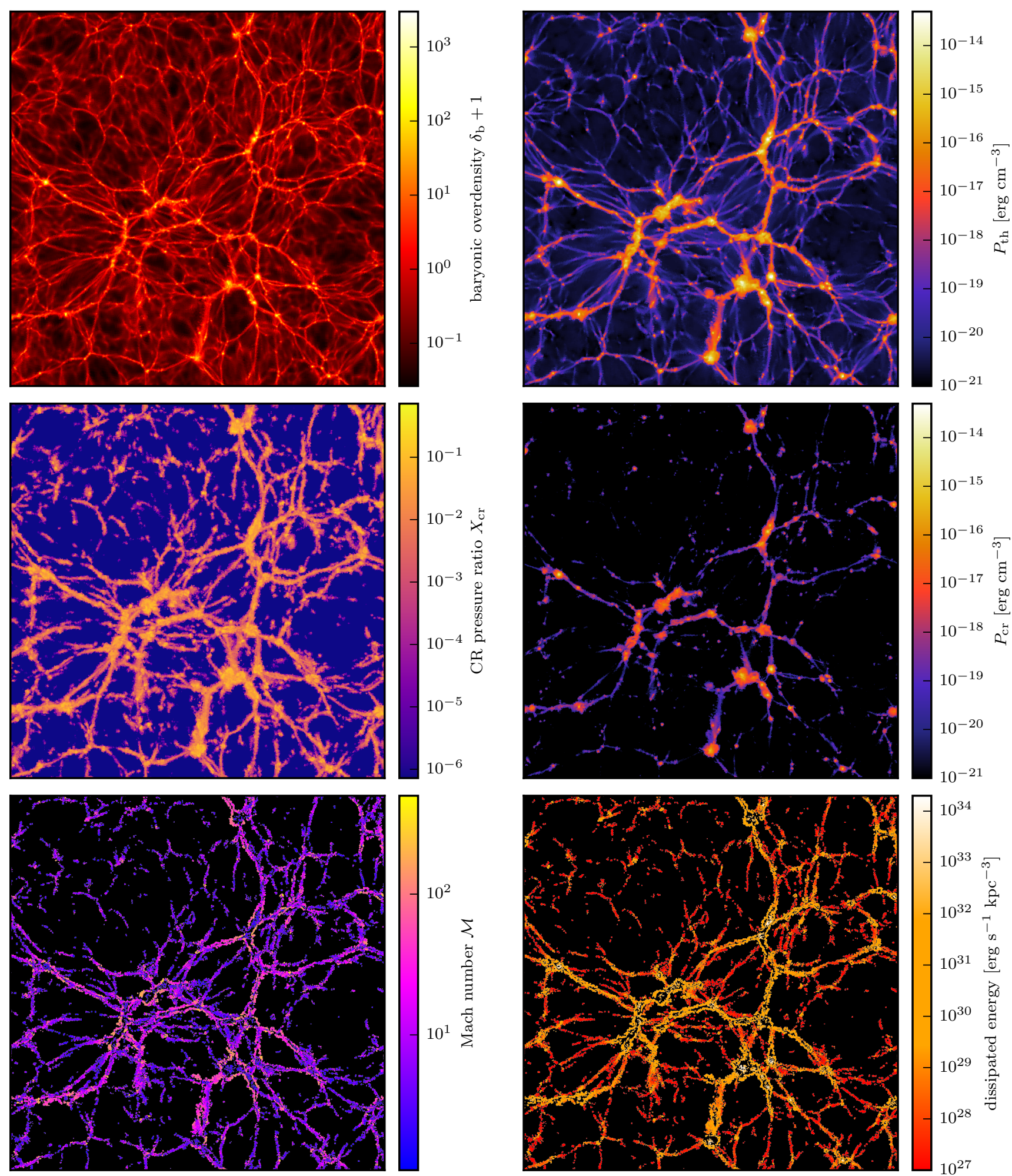

Figure 8. Visualisation of a non-radiative cosmological MHD simulation at redshift $z=0$ which accounts for CR acceleration at structure formation shocks. Top panels: projections of the volume-weighted baryonic overdensity and the thermal pressure. The width and the height of the plots correspond to the full box size $\left(100 \mathrm{~h}^{-1} \mathrm{Mpc}\right)$. All projections exhibit a depth of $300 \mathrm{kpc}$. Middle panels: projection of the CR pressure ratio $X_{\mathrm{cr}}=P_{\mathrm{cr}} / P_{\mathrm{th}}$ and the volume-weighted CR pressure. Bottom panels: projections of the Mach number of structure formation shocks weighted with the energy dissipation (left-hand panel) and energy dissipation rate density (right-hand panel). 


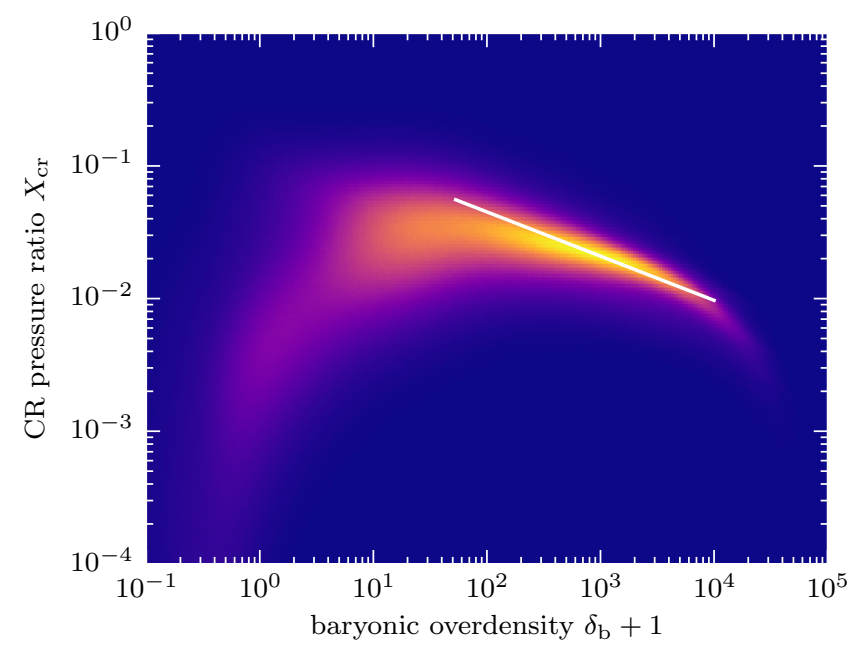

Figure 9. Distribution of the CR pressure ratio $X_{\mathrm{cr}}=P_{\mathrm{cr}} / P_{\mathrm{th}}$ as a function of baryonic overdensity, $\delta_{\mathrm{b}}$, at $z=0$. The colours show a linear scaling of the phase space density. CRs are accelerated at strong external formation shocks that form at overdensities of $\delta_{\mathrm{b}} \sim 5$ to 50. During the collapse into haloes, the composite of CRs and thermal gas experiences adiabatic compression, which favours the thermal pressure over that provided by CRs and causes the CR pressure ratio to drop as $X_{\mathrm{cr}} \propto \rho^{-1 / 3}$ (white line).

This can be understood by looking at the distribution of the CR pressure ratio $X_{\mathrm{cr}}=P_{\mathrm{cr}} / P_{\mathrm{th}}$ as a function of baryonic overdensity (Fig. 9) in combination with spherical collapse theory. As an overdensity collapses under the influence of its own self-gravity, its expansion rate drops below the Hubble expansion and its overdensity starts to increase (while its physical density continues to decrease with the Hubble expansion). As the overdensity reaches a value of $\delta_{\mathrm{b}}=5.55$ (in the spherical collapse model), the expanding shell of pristine cosmic gas turns around and collapses onto filaments and super-cluster regions. As a result, cosmological formation shocks form at the location where the ram pressure of the collapsing gas, $\rho v^{2}$, balances the total pressure, $P_{\mathrm{tot}}=P_{\mathrm{th}}+P_{\mathrm{cr}}$, of the previously collapsed shells of gas and the cold pristine gas is shock-heated for the first time in cosmic history. In our simulations, these shocks are characterised by overdensities of $\delta_{\mathrm{b}} \sim 5$ to 50, and the CR pressure ratio in the shock-heated gas amounts to $X_{\text {cr }} \lesssim 0.05$, as expected for our injection efficiency and cold pre-shock gas. During the continued collapse into haloes, the composite of CRs and thermal gas is adiabatically compressed, which favours the thermal pressure over the $\mathrm{CR}$ pressure and hence causes the $\mathrm{CR}$ pressure ratio to drop as $X_{\text {cr }} \propto \rho^{-1 / 3}$. Finally, at the highest densities, the Coulomb and hadronic cooling time scales are shorter than the Hubble time and CRs start to loose pressure support at overdensities of $\delta_{\mathrm{b}} \gtrsim 10^{4}$. The density dependence of $X_{\mathrm{cr}}$ nicely resembles the smoothed particle hydrodynamics results by Pfrommer et al. (2007) while the decline of $X_{\text {cr }}$ as a result of adiabatic compression in the adaptive-mesh refinement simulations by Vazza et al. (2014) is virtually absent and converges only very weakly with increasing resolution.

Figure 10 quantifies the shock distribution and the associated energy dissipation in our simulation. The left-hand panel shows the differential shock surface area per unit vol- ume as a function of Mach number for different redshifts that are equally spaced in look-back time. Note that in our analysis we neither account for radiative cooling nor for reionisation. We find that the cumulative area of shocks is dominated by weak shocks with Mach numbers $\mathcal{M}_{1}=3$ to 4 and decreases towards lower redshift, while the surface area increases for strong shocks with $\mathcal{M}_{1} \gtrsim 20$. The trend of the shock area with redshift is reversed for weak shocks if the pre-shock gas is assumed to be photo-heated to $10^{4} \mathrm{~K}$, in which case we reproduce the result found by Schaal \& Springel (2015). Essentially, such an analysis increases the Jeans mass in voids above the masses of most of the haloes so that shocks would not have formed in voids in such a realisation of the universe. Instead, we show here the unmodified shock distribution, which was responsible for the CR acceleration our simulations. At low redshift, the accretion from previously unshocked gas onto hot filaments and cluster outskirts forms shocks with Mach numbers of $\mathcal{M}_{1} \sim 100$, which decisively changes the slope of the Mach number distribution and introduces a shoulder around $\mathcal{M}_{1} \sim 100$ at late times.

The right-hand panel of Fig. 10 shows the distribution of dissipated energy density per time, which includes the generated thermal and CR energy at the shock. Most importantly, CR shock acceleration does not change the overall appearance of the shock distribution, which is shaped by the distribution of gravitational potentials in space and potential depths. Theoretically, we would expect the Mach numbers to be slightly weaker in the case with $\mathrm{CR}$ acceleration but this difference is not visible on the large logarithmic scale in Fig. 10 (it is only a $5 \%$ effect at $\mathcal{M}_{1}=10$ for an extreme CR injection efficiency of $\zeta=0.5$ ). The total dissipated energy increases with time until $z=0.5$ and slightly decreases thereafter. The increase of the shock-dissipated energy with time is due to the increasing densities in collapsed structures and increasing shock velocities as deeper potential wells are forming, since the kinetic energy flux through the shock scales as $F_{\text {kin }} \propto \rho v_{\mathrm{s}}^{3}$. At low redshifts, this effect saturates because of the self-similar density profiles of collapsed haloes in nonradiative simulations and, furthermore, dark energy slows structure growth and dilutes the pre-shock gas inside voids, which leads to a drop of the thermal energy flux for high Mach numbers (Pfrommer et al. 2006).

To test for numerical convergence, we additionally simulated the same cosmological model by degrading the particle resolution in steps of 8 . We find convergence in the Mach number distributions at late times $z<3$, indicating that the bulk of the relevant shock structures are well resolved.

\section{SUMMARY AND CONCLUSIONS}

In this paper, we have detailed a new versatile approach for treating CR physics coupled to MHD in a hydrodynamical code with an unstructured moving mesh, as realised in the massively parallel AREPO code. This enables us to perform self-consistent MHD-CR simulations in a cosmological framework. We model the CR distribution as a second fluid with an adiabatic index (usually taken to be $\gamma_{\mathrm{cr}}=4 / 3$ ). Our implementation accounts for diffusive shock acceleration of CRs at resolved shocks in the computational domain, and additionally from supernova remnants that are individually not resolved in simulations of galaxy formation, but that fol- 

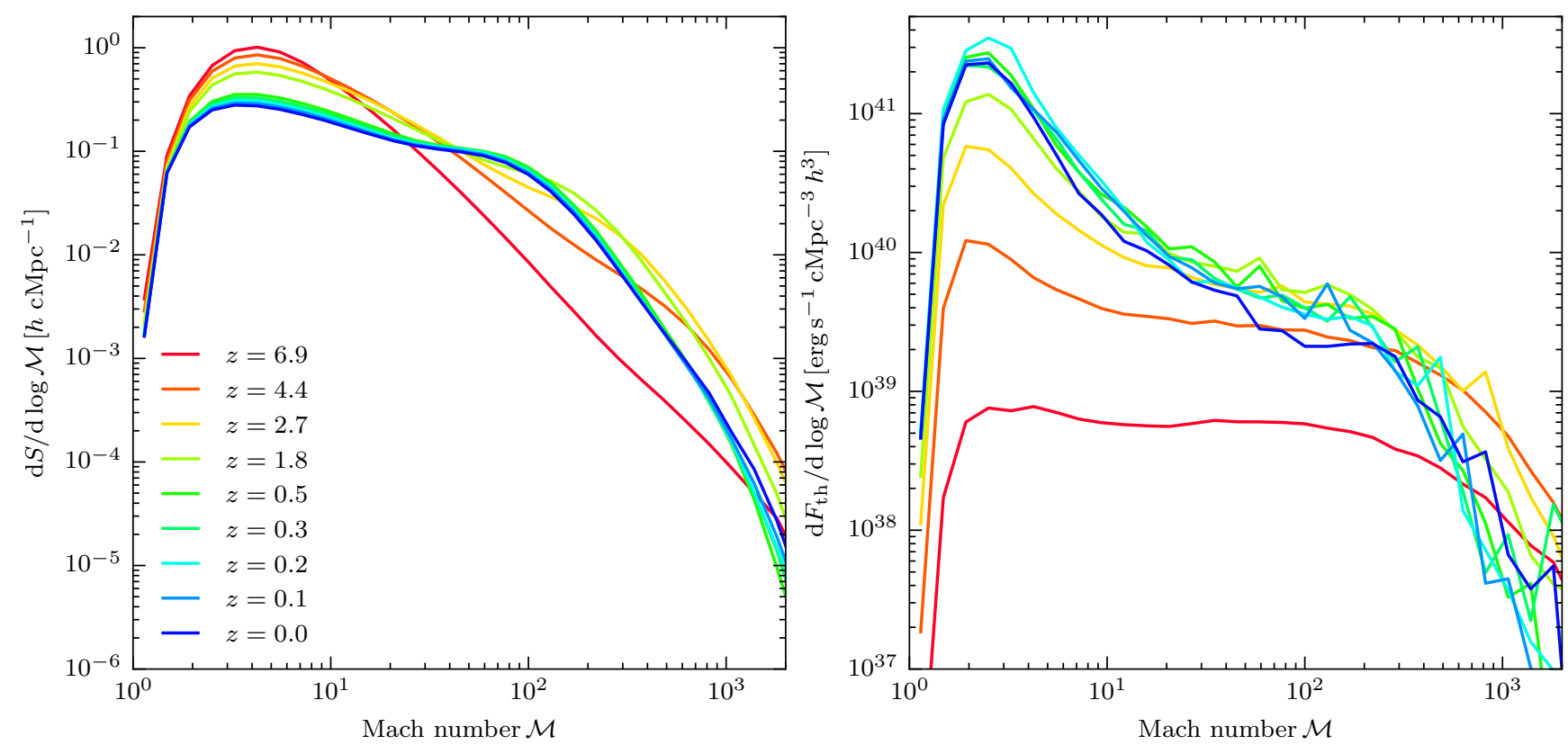

Figure 10. Left-hand panel: we show the differential shock surface area per unit volume as a function of Mach number for different redshifts. Right-hand panel: distribution of the dissipated energy density per time, which accounts for the total of generated thermal and CR energy at the shock. While there is more energy dissipated at late times in weak internal shocks, the shock surface per volume decreases for weak shocks.

low star formation and feedback in a sub-resolution framework.

So far, our approach follows the advective transport of CRs with the magnetised plasma as well as the anisotropic diffusive transport along the orientation of the local magnetic field lines. For the latter, we employ a gradient-limited, conservative, semi-implicit scheme for anisotropic CR diffusion that supports local time stepping, as described in a companion paper (Pakmor et al. 2016b). We account for the most important CR loss processes in the form of Coulomb and hadronic interactions with the thermal plasma by adopting an equilibrium CR distribution that results from balance between injection and dissipation processes. Furthermore, we also model CR energy losses as a result of the generation of Alfvén waves by the CR streaming instability. This novel element enables us to study problems associated with $\mathrm{CR}$ acceleration at supernova remnants, and to understand the dynamical impact of CRs on galaxy formation and the evolution of galaxy clusters.

We have validated our new numerical methods in a number of different problem set-ups, including CR acceleration at planar and spherically expanding shocks, isolated galaxy formation, and CR acceleration and transport in nonradiative cosmological simulations.

- Riemann shock tube. We demonstrate the accuracy of our CR implementation in a Riemann shock tube problem with a pre-existing $\mathrm{CR}$ population that modifies the dynamics, but - in the absence of CR shock acceleration - is only adiabatically compressed across the shock jump. To model diffusive shock acceleration at shocks in our simulation, we find and characterise them on-the-fly and accelerate CRs according to a sub-resolution prescription from PIC plasma simulations. Our shock tube simulations com- pare very well to newly derived, exact solutions of the Riemann shock-tube problem with $\mathrm{CR}$ acceleration (as shown in the Appendices B and C). As expected, injecting and accelerating CRs from the thermal distribution lowers the effective adiabatic index of the post-shock gas, increases its compressibility and causes it to become denser. Because of mass conservation and of the higher post-shock gas density, the shock does then not propagate as fast as in the case without $\mathrm{CR}$ acceleration.

- Sedov-Taylor blast wave. A slower propagating shock in the case of diffusive shock acceleration of CRs is also seen for a spherically expanding shock as a result of a point explosion, corresponding to the well-known SedovTaylor problem. We observe a strongly varying effective adiabatic index $\gamma_{\text {eff }}$ of the two-fluid medium as a result of CR shock acceleration: the value of $\gamma_{\text {eff }}$ decreases from its canonical value of $5 / 3$ in the external medium to a lower value that depends on the CR shock-acceleration efficiency because the freshly accelerated CRs exhibit a softer equation of state. Importantly, $\gamma_{\text {eff }}$ drops further to a value close to that of the intrinsic CR population of $\gamma_{\mathrm{cr}} \approx 4 / 3$ because of the successive adiabatic expansion in the interior of the blast wave that favours the CR pressure over the thermal pressure. Despite this strong variation of the adiabatic index, the selfsimilarity of the solution is almost conserved. In particular, the evolution of the expanding shock radius as well as the detailed pressure and density profiles can be well approximated by a constant but modified adiabatic index which we find to be close to $7 / 5$ for our optimistic CR acceleration efficiency of $\zeta=0.5$.

- Galaxy formation. To explore the impact of $\mathrm{CR}$ physics on MHD simulations of galaxy formation, we model $\mathrm{CR}$ injection at supernovae, advective $\mathrm{CR}$ transport, and Coulomb and hadronic CR interactions with the ambient 
gas. In line with previous findings that employed smoothed particle hydrodynamics, we find that CR pressure feedback suppresses star formation more strongly in smaller galaxies in comparison to simulations without CRs. During the first starburst, the CR energy density in the disk quickly reaches equilibrium with the thermal energy density and dominates the internal energy budget soon thereafter. The slowly cooling non-thermal pressure reservoir provided by CRs causes the disks to be more expanded in the vertical direction, providing additional dynamical stability to a disk that would otherwise be unstable to gravitational collapse.

The local injection of CR energy at supernovae modifies the multi-phase structure of the ISM, which exhibits an amorphous clumpy structure when CR physics is included. Without CRs, this multiphase structure is mostly suppressed by the stiff effective equation of state above of the adopted subgrid model for star formation and its regulation. We envision that such a multiphase structure as a result of local $\mathrm{CR}$ feedback will also be maintained when we additionally account for CR streaming and diffusion, and employ a temporarily and spatially varying diffusion coefficient. The latter is expected to increase with distance from the location of supernova remnants and reach the average Galactic value on large scales.

Initially, all our galaxy models with halo masses ranging from $10^{10}$ to $10^{12} \mathrm{M}_{\odot}$ exhibit a rapid turbulent dynamo for the magnetic field that eventually transitions to a slower amplification process. This also increases the magnetic coherence scale of the field as it is wound up by differential rotation of the disk. The field structure is very regular and quiet in the simulations without CRs, reflecting the pressurised equation of state of the ISM. In contrast, in our CR simulations, it attains a chaotic small-scale component which is superimposed on the dominant azimuthal structure. We find that the additional turbulent velocity field is a result of supernova explosions that sustain an additional dynamo action, amplifying the field to observed strengths exceeding $10 \mu \mathrm{G}$ in the centres of the disks.

- Cosmological simulations. To study the impact of diffusive shock acceleration on cosmological structure formation shocks, we have simulated a representative volume of the universe with the currently favoured $\Lambda$ CDM cosmology, following non-radiative MHD. By employing our on-the-fly shock finder, we model CR acceleration at formation shocks that exceed pre-shock Mach numbers $\mathcal{M}_{1}=3$, and subsequently followed their advective transport as well as their Coulomb and hadronic interactions with the ambient gas. We find that CRs do not modify the shock statistics, which is shaped by the distribution of gravitational potentials in space and potential depths. CRs are mostly accelerated at strong external formation shocks that form at overdensities of $\delta_{\mathrm{b}} \sim 5$ to 50 as the pristine cosmic fluid collapses onto filaments and sheets. During the collapse into haloes, the composite of CRs and thermal gas experiences adiabatic compression, which increases both pressure components. However, this favours the thermal pressure over that provided by CRs and causes the CR pressure ratio to drop with density as $X_{\text {cr }} \propto \rho^{-1 / 3}$. At the highest densities, the Coulomb and hadronic cooling time scales are shorter than the Hubble time, and CRs start to loose pressure support at overdensities of $\delta_{\mathrm{b}} \gtrsim 10^{4}$.
Combined with our companion paper on the technical details of the anisotropic CR transport solver on a moving unstructured mesh (Pakmor et al. 2016b), we have introduced a new advanced treatment of CR physics in current cosmological hydrodynamic codes. We expect this will be useful for studying many timely questions related to CR physics and galaxy formation. In Pakmor et al. (2016c) we have already studied wind formation in disk galaxies, and in Simpson et al. (2016) explored the problem of star formation regulation in high-resolution simulations of the ISM. It will be very interesting to extend these works to further science applications.

\section{ACKNOWLEDGEMENTS}

The authors would like to thank the referee for a constructive report which helped improving this paper. This work has been supported by the European Research Council under ERC-CoG grant CRAGSMAN-646955, ERC-StG grant EXAGAL-308037 and by the Klaus Tschira Foundation. VS and KS acknowledge support through subproject EXAMAG of the Priority Programme 1648 'Software for Exascale Computing' of the German Science Foundation.

\section{REFERENCES}

Achatz U., Steinacker J., Schlickeiser R., 1991, A\&A, 250, 266

Ackermann M., et al. 2013, Science, 339, 807

Battaglia N., Bond J. R., Pfrommer C., Sievers J. L., 2012a, ApJ, 758,74

Battaglia N., Bond J. R., Pfrommer C., Sievers J. L., 2012b, ApJ, 758,75

Battaglia N., Bond J. R., Pfrommer C., Sievers J. L., 2013, ApJ, 777,123

Bauer A., Springel V., 2012, MNRAS, 423, 2558

Bell A. R., 2004, MNRAS, 353, 550

Booth C. M., Agertz O., Kravtsov A. V., Gnedin N. Y., 2013, ApJ, 777, L16

Boulares A., Cox D. P., 1990, ApJ, 365, 544

Breitschwerdt D., McKenzie J. F., Voelk H. J., 1991, A\&A, 245, 79

Breitschwerdt D., Dogiel V. A., Völk H. J., 2002, A\&A, 385, 216 Brunetti G., Jones T. W., 2014, Int. J. Mod. Phys. D, 23, 1430007

Caprioli D., Spitkovsky A., 2014a, ApJ, 783, 91

Caprioli D., Spitkovsky A., 2014b, ApJ, 794, 46

Caprioli D., Pop A.-R., Spitkovsky A., 2015, ApJ, 798, L28

Courant R., Friedrichs K. O., 1948, Supersonic flow and shock waves. Pure and Applied Mathematics, New York: Interscience, 1948

Di Matteo T., Springel V., Hernquist L., 2005, Nature, 433, 604

Dolag K., Komatsu E., Sunyaev R., 2016, MNRAS,

Donnert J., Dolag K., Brunetti G., Cassano R., 2013, MNRAS, 429,3564

Dorfi E. A., Breitschwerdt D., 2012, A\&A, 540, A77

Enßlin T. A., Pfrommer C., Springel V., Jubelgas M., 2007, A\&A, 473,41

Enßlin T., Pfrommer C., Miniati F., Subramanian K., 2011, A\&A, 527, A99

Everett J. E., Zweibel E. G., Benjamin R. A., McCammon D., Rocks L., Gallagher III J. S., 2008, ApJ, 674, 258

Everett J. E., Schiller Q. G., Zweibel E. G., 2010, ApJ, 711, 13

Farmer A. J., Goldreich P., 2004, ApJ, 604, 671

Fujita Y., Ohira Y., 2012, ApJ, 746, 53

Girichidis P., et al., 2016, ApJ, 816, L19 
Gould R. J., 1972, Physica, 58, 379

Guedes J., Callegari S., Madau P., Mayer L., 2011, ApJ, 742, 76

Guo F., Oh S. P., 2008, MNRAS, 384, 251

Hall D. E., Sturrock P. A., 1967, Physics of Fluids, 10, 2620

Hanasz M., Lesch H., Naab T., Gawryszczak A., Kowalik K., Wóltański D., 2013, ApJ, 777, L38

Helder E. A., Vink J., Bykov A. M., Ohira Y., Raymond J. C., Terrier R., 2012, Space Sci. Rev., 173, 369

Henriques B. M. B., White S. D. M., Thomas P. A., Angulo R., Guo Q., Lemson G., Springel V., Overzier R., 2015, MNRAS, 451, 2663

Hong S. E., Ryu D., Kang H., Cen R., 2014, ApJ, 785, 133

Hopkins P. F., Kereš D., Oñorbe J., Faucher-Giguère C.-A., Quataert E., Murray N., Bullock J. S., 2014, MNRAS, 445, 581

Ipavich F. M., 1975, ApJ, 196, 107

Jacob S., Pfrommer C., 2016a, preprint, (arXiv:1609.06321)

Jacob S., Pfrommer C., 2016b, preprint, (arXiv:1609.06322)

Jubelgas M., Springel V., Enßlin T., Pfrommer C., 2008, A\&A, 481,33

Kang H., Ryu D., Cen R., Ostriker J. P., 2007, ApJ, 669, 729

Kennicutt Jr. R. C., 1998, ApJ, 498, 541

Kravtsov A. V., Borgani S., 2012, ARA\&A, 50, 353

Kroupa P., 2001, MNRAS, 322, 231

Krumholz M. R., Thompson T. A., 2012, ApJ, 760, 155

Kulsrud R., Pearce W. P., 1969, ApJ, 156, 445

Lerche I., 1967, ApJ, 147, 689

Lloyd S., 1982, IEEE Trans Inf. Theory, 28, 129

Loewenstein M., Zweibel E. G., Begelman M. C., 1991, ApJ, 377, 392

Mannheim K., Schlickeiser R., 1994, A\&A, 286, 983

Marinacci F., Pakmor R., Springel V., 2014, MNRAS, 437, 1750

McCarthy I. G., Le Brun A. M. C., Schaye J., Holder G. P., 2014, MNRAS, 440, 3645

McCarthy I. G., Schaye J., Bird S., Le Brun A. M. C., 2016, preprint, (arXiv:1603.02702)

McKenzie J. F., Voelk H. J., 1982, A\&A, 116, 191

McNamara B. R., Nulsen P. E. J., 2007, ARA\&A, 45, 117

McNamara B. R., Nulsen P. E. J., 2012, New Journal of Physics, 14,055023

Miniati F., Ryu D., Kang H., Jones T. W., 2001a, ApJ, 559, 59

Miniati F., Jones T. W., Kang H., Ryu D., 2001b, ApJ, 562, 233

Miyoshi T., Kusano K., 2005, J. Comput. Phys., 208, 315

Morlino G., Caprioli D., 2012, A\&A, 538, A81

Murray N., Quataert E., Thompson T. A., 2005, ApJ, 618, 569

Navarro J. F., Frenk C. S., White S. D. M., 1997, ApJ, 490, 493

Oppenheimer B. D., Davé R., 2006, MNRAS, 373, 1265

Pakmor R., Springel V., 2013, MNRAS, 432, 176

Pakmor R., Bauer A., Springel V., 2011, MNRAS, 418, 1392

Pakmor R., Springel V., Bauer A., Mocz P., Munoz D. J., Ohlmann S. T., Schaal K., Zhu C., 2016a, MNRAS, 455, 1134

Pakmor R., Pfrommer C., Simpson C. M., Kannan R., Springel V., 2016b, MNRAS, 462, 2603

Pakmor R., Pfrommer C., Simpson C. M., Springel V., 2016c, ApJ, 824, L30

Peterson J. R., Fabian A. C., 2006, Phys. Rep., 427, 1

Pfrommer C., 2008, MNRAS, 385, 1242

Pfrommer C., 2013, ApJ, 779, 10

Pfrommer C., Enßlin T. A., 2004, A\&A, 413, 17

Pfrommer C., Springel V., Enßlin T. A., Jubelgas M., 2006, MNRAS, 367, 113

Pfrommer C., Enßlin T. A., Springel V., Jubelgas M., Dolag K., 2007, MNRAS, 378, 385

Pfrommer C., Enßlin T. A., Springel V., 2008, MNRAS, 385, 1211

Pinzke A., Pfrommer C., 2010, MNRAS, 409, 449

Pinzke A., Oh S. P., Pfrommer C., 2013, MNRAS, 435, 1061

Pinzke A., Oh S. P., Pfrommer C., 2015, preprint, (arXiv: 1503.07870)
Planck Collaboration et al., 2016, A\&A, 594, A13

Planelles S., Quilis V., 2013, MNRAS, 428, 1643

Powell K. G., Roe P. L., Linde T. J., Gombosi T. I., De Zeeuw D. L., 1999, J. Comput. Phys., 154, 284

Ptuskin V. S., Voelk H. J., Zirakashvili V. N., Breitschwerdt D., 1997, A\&A, 321, 434

Puchwein E., Springel V., 2013, MNRAS, 428, 2966

Recchia S., Blasi P., Morlino G., 2016, MNRAS, 462, 4227

Rephaeli Y., 1979, ApJ, 227, 364

Rodrigues L. F. S., Sarson G. R., Shukurov A., Bushby P. J., Fletcher A., 2016, ApJ, 816, 2

Rosdahl J., Schaye J., Teyssier R., Agertz O., 2015, MNRAS, 451, 34

Ruszkowski M., Yang H.-Y. K., Zweibel E., 2016, preprint, (arXiv: 1602.04856)

Ryu D., Kang H., Hallman E., Jones T. W., 2003, ApJ, 593, 599

Salem M., Bryan G. L., 2014, MNRAS, 437, 3312

Salem M., Bryan G. L., Hummels C., 2014, ApJ, 797, L18

Samui S., Subramanian K., Srianand R., 2010, MNRAS, 402, 2778

Sarazin C. L., 1999, ApJ, 520, 529

Schaal K., Springel V., 2015, MNRAS, 446, 3992

Schaal K., et al., 2016, MNRAS, 461, 4441

Schaye J., Dalla Vecchia C., 2008, MNRAS, 383, 1210

Schaye J., et al., 2010, MNRAS, 402, 1536

Schaye J., et al., 2015, MNRAS, 446, 521

Schlickeiser R., 2002, Cosmic Ray Astrophysics

Sedov L. I., 1959, Similarity and Dimensional Methods in Mechanics

Sijacki D., Pfrommer C., Springel V., Enßlin T. A., 2008, MNRAS, 387, 1403

Sijacki D., Vogelsberger M., Kereš D., Springel V., Hernquist L., 2012, MNRAS, 424, 2999

Simpson C. M., Pakmor R., Marinacci F., Pfrommer C., Springel V., Glover S. C. O., Clark P. C., Smith R. J., 2016, ApJ, 827, L29

Skilling J., 1971, ApJ, 170, 265

Skilling J., 1975, MNRAS, 172, 557

Skillman S. W., O'Shea B. W., Hallman E. J., Burns J. O., Norman M. L., 2008, ApJ, 689, 1063

Skinner M. A., Ostriker E. C., 2015, ApJ, 809, 187

Socrates A., Davis S. W., Ramirez-Ruiz E., 2008, ApJ, 687, 202 Springel V., 2010, MNRAS, 401, 791

Springel V., Hernquist L., 2003, MNRAS, 339, 289

Springel V., Di Matteo T., Hernquist L., 2005, MNRAS, 361, 776

Stage M. D., Allen G. E., Houck J. C., Davis J. E., 2006, Nature Physics, 2, 614

Takahashi K., Yamada S., Yamada 2014, Journal of Plasma Physics, 80, 255

Thompson T. A., Quataert E., Murray N., 2005, ApJ, 630, 167

Tüllmann R., Dettmar R.-J., Soida M., Urbanik M., Rossa J., 2000, A\&A, 364, L36

Uhlig M., Pfrommer C., Sharma M., Nath B. B., Enßlin T. A., Springel V., 2012, MNRAS, 423, 2374

Vazza F., Brunetti G., Gheller C., 2009, MNRAS, 395, 1333

Vazza F., Dolag K., Ryu D., Brunetti G., Gheller C., Kang H., Pfrommer C., 2011, MNRAS, 418, 960

Vazza F., Brüggen M., Gheller C., Brunetti G., 2012, MNRAS, 421,3375

Vazza F., Gheller C., Brüggen M., 2014, MNRAS, 439, 2662

Vogelsberger M., Sijacki D., Kereš D., Springel V., Hernquist L., 2012, MNRAS, 425, 3024

Vogelsberger M., et al., 2014, MNRAS, 444, 1518

Wiener J., Oh S. P., Guo F., 2013, MNRAS, 434, 2209

Wiener J., Pfrommer C., Oh S. P., 2016, preprint, (arXiv: 1608.02585)

Zhuravleva I., et al., 2014, Nature, 515, 85

Zirakashvili V. N., Breitschwerdt D., Ptuskin V. S., Voelk H. J., 1996, A\&A, 311, 113 


\section{APPENDIX A: COSMIC RAY HYDRODYNAMICS}

In this Appendix, we derive the energy equation for CRs in a magnetised plasma (McKenzie \& Voelk 1982; Guo \& Oh 2008), additionally augmented with an expression for momentum diffusion as a result of second-order Fermi acceleration. When CRs are streaming along the local magnetic field at a speed faster than the Alfvén speed, they resonantly excite Alfvén waves at the gyroscale by the CR streaming instability (Lerche 1967; Kulsrud \& Pearce 1969). These waves scatter the CRs in pitch angle and attempt to confine them to the frame comoving with the Alfvén waves. Here, we only consider forward Alfvén waves that propagate nearly parallel to the unperturbed background magnetic field, in the direction of the streaming CRs (backward Alfvén waves are damped; see Lerche 1967; Kulsrud \& Pearce 1969). Depending on the damping rate of the forward Alfvén waves due to turbulent damping or non-linear Landau damping in an ionised plasma (Farmer \& Goldreich 2004; Kulsrud \& Pearce 1969), and additional ion-neutral damping in a sufficiently neutral plasma, this confinement can be incomplete, leading to a diffusive motion relative to the forward Alfvén wave frame (Wiener et al. 2013).

Defining a dimensionless momentum of a particle, $\boldsymbol{p}=\boldsymbol{P}_{\mathrm{p}} /(m c)$ and its magnitude $p=|\boldsymbol{p}|$, we start with the relativistic Vlasov equation for the $3 \mathrm{D} \mathrm{CR}$ distribution function $f_{\mathrm{p}}$ and derive a Fokker-Planck equation for the transport of CRs (Skilling 1971, 1975; Schlickeiser 2002),

$$
\frac{\partial f_{\mathrm{p}}}{\partial t}+\left(\boldsymbol{v}+\boldsymbol{v}_{\mathrm{st}}\right) \cdot \boldsymbol{\nabla} f_{\mathrm{p}}=\boldsymbol{\nabla} \cdot\left[\kappa_{\mathrm{p}} \boldsymbol{b}\left(\boldsymbol{b} \cdot \boldsymbol{\nabla} f_{\mathrm{p}}\right)\right]+\frac{1}{3} p \frac{\partial f_{\mathrm{p}}}{\partial p} \boldsymbol{\nabla} \cdot\left(\boldsymbol{v}+\boldsymbol{v}_{\mathrm{st}}\right)+\frac{1}{p^{2}} \frac{\partial}{\partial p}\left[p^{2} \Gamma_{\mathrm{p}} \frac{\partial f_{\mathrm{p}}}{\partial p}\right]+Q_{\mathrm{p}},
$$

where $f_{\mathrm{p}}=f_{\mathrm{p}}(\boldsymbol{x}, p, t)$ is the isotropic momentum part of the CR phase space distribution function. This equation has been derived in the quasi-linear approximation that assumes small-amplitude electro-magnetic fluctuations. It requires incoherent mode coupling of the fluctuating electromagnetic fields described as the superposition of individual plasma wave modes. It is only valid on timescales long compared to the pitch angle scattering relaxation time $\tau \sim O\left(D_{\mu \mu}^{-1}\right)$ where $D_{\mu \mu}=D_{\mu \mu}(\boldsymbol{x}, p, \mu)$ is the Fokker-Planck coefficient representing the frequency of pitch angle scattering of CRs by hydromagnetic waves. Here $\mu \equiv \boldsymbol{b} \cdot \boldsymbol{p} / p$ denotes the pitch-angle cosine, and $\boldsymbol{b}=\boldsymbol{B} /|\boldsymbol{B}|$ is a unit vector along the local magnetic field.

Under these conditions, the particles can locally reach near-equilibrium, which results in a small anisotropy of the distribution function, i.e. $\delta f_{\mathrm{p}} \ll f_{\mathrm{p}}$. Note that equation (A1) employs a mixed coordinate frame in which the configuration-space coordinates $(\boldsymbol{x})$ are measured in the laboratory system and the momentum-space coordinates $\boldsymbol{p}=\left(p \sqrt{1-\mu^{2}} \cos \varphi, p \sqrt{1-\mu^{2}} \sin \varphi, p \mu\right)$ are defined with respect to the rest frame of the streaming CRs, i.e., in the frame comoving with the velocity $\boldsymbol{v}+\boldsymbol{v}_{\mathrm{st}}$. In this equation, $\boldsymbol{v}$ is the mean velocity of the thermal background plasma, $\boldsymbol{v}_{\mathrm{st}}=-\boldsymbol{v}_{\mathrm{A}} \operatorname{sgn}\left(\boldsymbol{B} \cdot \boldsymbol{\nabla} f_{\mathrm{p}}\right)$ is the streaming velocity of CRs, $\boldsymbol{v}_{\mathrm{A}}=\boldsymbol{B} / \sqrt{4 \pi \rho}$ is the local Alfvén velocity (in the cgs system of units), and $\boldsymbol{\nabla} \equiv \partial / \partial \boldsymbol{x}$. The spatial diffusion coefficient $\kappa_{\mathrm{p}}$ (in units of $\mathrm{cm}^{2} \mathrm{~s}^{-1}$ ) and the momentum diffusion rate $\Gamma_{\mathrm{p}}$ (in units of $\mathrm{s}^{-1}$ ) are given by

$$
\kappa_{\mathrm{p}}(x, p)=\frac{\beta^{2} c^{2}}{8} \int_{-1}^{+1} \frac{\left(1-\mu^{2}\right)^{2}}{D_{\mu \mu}} \mathrm{d} \mu, \quad \text { and } \quad \Gamma_{\mathrm{p}}=\frac{1}{2} \int_{-1}^{+1}\left[D_{p p}-\frac{D_{\mu p}^{2}}{D_{\mu \mu}}\right] \mathrm{d} \mu,
$$

where $\beta=p / \gamma$ is the dimensionless CR particle speed and $\gamma=\sqrt{1+p^{2}}$ is its Lorentz factor. Here $D_{p p}=D_{p p}(\boldsymbol{x}, p, \mu)$ and $D_{\mu p}=D_{\mu p}(x, p, \mu)$ are the Fokker-Planck coefficients representing the ensemble averages of the rate of change of the particles' momenta $p$ with themselves and with the rate of change of the particles' pitch angles $\mu$, evaluated along the first-order corrections to the particle orbits (Hall \& Sturrock 1967; Achatz et al. 1991),

$$
D_{\mu \mu}=\mathcal{R} \int_{0}^{\infty}\left\langle\dot{\mu}(t) \dot{\mu}^{*}(t+\tau)\right\rangle \mathrm{d} \tau, \quad D_{\mu p}=\mathcal{R} \int_{0}^{\infty}\left\langle\dot{\mu}(t) \dot{p}^{*}(t+\tau)\right\rangle \mathrm{d} \tau, \quad D_{p p}=\mathcal{R} \int_{0}^{\infty}\left\langle\dot{p}(t) \dot{p}^{*}(t+\tau)\right\rangle \mathrm{d} \tau .
$$

Here, the asterisk denotes the complex conjugate, $\mathcal{R}$ denotes the real part of the integral, and the equation of motion is

$$
\dot{\boldsymbol{p}}=Z e\left[\delta \boldsymbol{E}+\frac{1}{m c \gamma} \boldsymbol{p} \times\left(\boldsymbol{B}_{0}+\delta \boldsymbol{B}\right)\right],
$$

where $Z e$ denotes the charge of the particle, and $(\delta \boldsymbol{E}, \delta \boldsymbol{B})$ denote the fluctuations of the electromagnetic field with respect to the mean magnetic field $\boldsymbol{B}_{0}$. The term on the left-hand side of equation (A1) accounts for advective transport of the CR distribution function with the Alfvén wave frame relative to the laboratory rest frame, while the terms on the right-hand side represent, from left to right: diffusive transport along magnetic field lines, diffusive shock (first-order Fermi) acceleration, second-order Fermi acceleration (which is equivalent to momentum-space diffusion), and sources and sinks for the distribution function (generally denoted by $Q_{\mathrm{p}}$ ).

To derive the evolution equation for the CR number and energy density, we define three thermodynamic quantities as moments of $f_{\mathrm{p}}$, namely CR number density $n_{\mathrm{cr}}$, CR pressure $P_{\mathrm{cr}}$, and CR energy density $\varepsilon_{\mathrm{cr}}$ :

$$
\begin{aligned}
& n_{\mathrm{cr}}=4 \pi \int_{0}^{\infty} p^{2} f_{\mathrm{p}}(p) \mathrm{d} p=\frac{4 \pi C}{\alpha-3} q^{3-\alpha}, \\
& P_{\text {cr }}=\frac{4 \pi m c^{2}}{3} \int_{0}^{\infty} \beta p^{3} f_{\mathrm{p}}(p) \mathrm{d} p=\frac{4 \pi C m c^{2}}{6} \mathcal{B}_{\frac{1}{1+q^{2}}}\left(\frac{\alpha-4}{2}, \frac{5-\alpha}{2}\right), \\
& \varepsilon_{\mathrm{cr}}=4 \pi \int_{0}^{\infty} p^{2} E_{\mathrm{p}}(p) f_{\mathrm{p}}(p) \mathrm{d} p=\frac{4 \pi C m c^{2}}{\alpha-3}\left[\frac{1}{2} \mathcal{B}_{\frac{1}{1+q^{2}}}\left(\frac{\alpha-4}{2}, \frac{5-\alpha}{2}\right)+q^{3-\alpha}\left(\sqrt{1+q^{2}}-1\right)\right] .
\end{aligned}
$$

Here $\mathcal{B}_{x}(a, b)$ denotes the incomplete beta function (assuming $\alpha>4$ ) and $E_{\mathrm{p}}(p)$ is the kinetic energy of a CR particle with 
momentum $p$,

$$
E_{\mathrm{p}}(p)=\left(\sqrt{1+p^{2}}-1\right) m c^{2}
$$

For the explicit forms of equations (A5) to (A7), we adopted a power-law CR distribution function,

$$
f_{\mathrm{p}}(x, p, t) \equiv \frac{\mathrm{d} N}{\mathrm{~d}^{3} p \mathrm{~d} V}=C p^{-\alpha} \theta(p-q),
$$

with low-momentum cutoff $q$, normalisation $C$ and 3D spectral index $\alpha .{ }^{11}$

Integration of equation (A1) over all particle momenta yields the evolution equation for the CR number density $n_{\mathrm{cr}}$ :

$$
\frac{\partial n_{\mathrm{cr}}}{\partial t}+\boldsymbol{\nabla} \cdot \boldsymbol{F}_{n}=\bar{Q}_{n}, \quad \text { where } \quad \boldsymbol{F}_{n}=\left(\boldsymbol{v}+\boldsymbol{v}_{\mathrm{st}}\right) n_{\mathrm{cr}}-\kappa_{n} \boldsymbol{b}\left(\boldsymbol{b} \cdot \boldsymbol{\nabla} n_{\mathrm{cr}}\right) .
$$

Here, $\kappa_{n}$ is the momentum-space averaged spatial diffusion coefficient and $\bar{Q}_{n}$ is the net source of CRs, given by

$$
\kappa_{n}=\frac{\int_{0}^{\infty} p^{2} \kappa_{\mathrm{p}}(\boldsymbol{x}, p)\left(\boldsymbol{b} \cdot \boldsymbol{\nabla} f_{\mathrm{p}}\right) \mathrm{d} p}{\int_{0}^{\infty} p^{2}\left(\boldsymbol{b} \cdot \boldsymbol{\nabla} f_{\mathrm{p}}\right) \mathrm{d} p}, \quad \text { and } \quad \bar{Q}_{n}=4 \pi \int_{0}^{\infty} p^{2} Q_{\mathrm{p}}(\boldsymbol{x}, p) \mathrm{d} p .
$$

Note that the second-order Fermi acceleration term (with the rate coefficient $\Gamma_{\mathrm{p}}$ ) drops from this equation because this process conserves CR particle number. For this to hold mathematically, we have to require that the combination $\Gamma_{\mathrm{p}} \partial f_{\mathrm{p}} / \partial p$ vanishes at infinity in momentum space.

Multiplication of equation (A1) by $E_{\mathrm{p}}(p)$ and integration over all particle momenta results in an evolution equation for the CR energy density,

$$
\frac{\partial \varepsilon_{\mathrm{cr}}}{\partial t}+\boldsymbol{\nabla} \cdot \boldsymbol{F}_{\varepsilon}=\left(\boldsymbol{v}+\boldsymbol{v}_{\mathrm{st}}\right) \cdot \boldsymbol{\nabla} P_{\mathrm{cr}}+\Gamma_{\mathrm{acc}}+\bar{Q}_{\varepsilon}, \quad \text { where } \quad \boldsymbol{F}_{\varepsilon}=\left(\boldsymbol{v}+\boldsymbol{v}_{\mathrm{st}}\right)\left(P_{\mathrm{cr}}+\varepsilon_{\mathrm{cr}}\right)-\kappa_{\varepsilon} \boldsymbol{b}\left(\boldsymbol{b} \cdot \boldsymbol{\nabla} \varepsilon_{\mathrm{cr}}\right)
$$

Here $\kappa_{\varepsilon}$ is the kinetic energy-weighted spatial diffusion coefficient, $\bar{Q}_{\varepsilon}$ is the net source of mean kinetic energy density of CRs, and $\Gamma_{\text {acc }}$ is the gain rate of energy density due to second-order Fermi acceleration in units of $\mathrm{erg} \mathrm{cm}^{-3} \mathrm{~s}^{-1}$ :

$$
\begin{aligned}
\kappa_{\varepsilon} & =\frac{\int_{0}^{\infty} p^{2} E_{\mathrm{p}}(p) \kappa_{\mathrm{p}}(\boldsymbol{x}, p)\left(\boldsymbol{b} \cdot \boldsymbol{\nabla} f_{\mathrm{p}}\right) \mathrm{d} p}{\int_{0}^{\infty} p^{2} E_{\mathrm{p}}(p)\left(\boldsymbol{b} \cdot \boldsymbol{\nabla} f_{\mathrm{p}}\right) \mathrm{d} p}, \\
\bar{Q}_{\varepsilon} & =4 \pi \int_{0}^{\infty} p^{2} E_{\mathrm{p}}(p) Q_{\mathrm{p}}(\boldsymbol{x}, p) \mathrm{d} p, \text { and } \\
\Gamma_{\mathrm{acc}} & =-4 \pi m c^{2} \int_{0}^{\infty} \beta p^{2} \Gamma_{\mathrm{p}} \frac{\partial f_{\mathrm{p}}}{\partial p} \mathrm{~d} p>0 \text { for } \frac{\partial f_{\mathrm{p}}}{\partial p}<0 .
\end{aligned}
$$

The flux function $\boldsymbol{F}_{\varepsilon}$ of equation (A12) represents the advective transport of CR enthalpy density $\left(h_{\mathrm{cr}}=P_{\mathrm{cr}}+\varepsilon_{\mathrm{cr}}\right)$ with the total velocity $\left(\boldsymbol{v}+\boldsymbol{v}_{\mathrm{st}}\right)$ as well as the anisotropic diffusive transport of CR energy density into and out of a given volume element. The first term on the right-hand side describes the energy-loss rate of CRs due to the volume work of the CR pressure gradient on the background plasma $\left(\boldsymbol{v} \cdot \boldsymbol{\nabla} P_{\mathrm{cr}}\right)$ and the generation of Alfvén waves $\left(\boldsymbol{v}_{\mathrm{st}} \cdot \boldsymbol{\nabla} P_{\mathrm{cr}}\right) . \Gamma_{\mathrm{acc}}$ accounts for energy gain due to second-order Fermi acceleration (which is only a positive gain process if $\partial f_{\mathrm{p}} / \partial p<0$ ) and $\bar{Q}_{\varepsilon}=\Gamma_{\mathrm{cr}}+\Lambda_{\mathrm{cr}}$ represents various gain and loss processes for the CR energy density. Equation (A12) is mathematically equivalent to the fourth row of the matrix equation (1) employed in our code (where we subsumed $\Gamma_{\text {acc }}$ into $\Gamma_{\mathrm{cr}}$ for clarity of the notation). To first approximation, we will adopt a constant spatial diffusion coefficient so that $\kappa_{\mathrm{p}}=\kappa_{\varepsilon}$. Future work will employ a momentum dependence of $\kappa_{\mathrm{p}}$ so that the energy-weighted spatial diffusion coefficient automatically acquires a spatial dependence through the gradient of the distribution function $\left(\boldsymbol{\nabla} f_{\mathrm{p}}\right)$ in equation (A13). Omission of this spatial dependence will lead to results that are not self-consistent.

\section{APPENDIX B: RIEMANN SHOCK-TUBE PROBLEM WITH COSMIC RAY ACCELERATION}

Exact solvers of the Riemann shock-tube problem are of eminent importance for understanding the hydrodynamic behaviour of a fluid and for validating numerical implementations of approximate Riemann solvers. While exact solutions to the problem have been put forward for a single polytropic fluid (Courant \& Friedrichs 1948), for a MHD fluid (Takahashi et al. 2014), and a two-component fluid composed of CRs and thermal gas (Pfrommer et al. 2006), such an exact solution of the Riemann problem with CR acceleration at shocks is still lacking.

Collisionless shocks in astrophysical plasmas are able to accelerate thermal ions through the process of diffusive shock acceleration. The presence of freshly injected CRs modifies the classical Rankine-Hugoniot jump conditions due to the softer equation of state of CRs, which leads to a more compressible composite gas in the post-shock regime and thus an enhanced

11 Note that it is not required to specify the form of the CR distribution function for the derivation of the CR energy equation. However, we will employ such a simplified form for the distribution function of the injected CR population when we derive the CR cooling rates in Sect. 3.3. 
density jump compared to the classical case of a purely thermal gas. In reality, CRs are diffusing multiple times across the shock front and develop a precursor in the upstream that adiabatically heats the incoming fluid before it encounters the discontinuity at the subshock. While it is possible for the highest-energy CRs to escape upstream of the shock, the majority - and in particular the pressure-carrying CRs with energies $E \sim m c^{2}$ - are swept downstream of the shock. Since we are interested in scales much larger than the diffusion length, which is given by the spatial extent of the CR precursor that we do not aim to resolve, we represent the shock region by a discontinuity and assume that the freshly accelerated CRs are injected into the downstream region of the shock.

The mathematical complexity of the solution differs depending on the presence of CRs in the initial conditions. Hence, in this section we first present the exact solution for the Riemann problem in the case of a polytropic gas $\left(\right.$ i.e., $\left.P_{\mathrm{th}}=(\gamma-1) \varepsilon_{\mathrm{th}}\right)$ experiencing a collisionless shock that is sufficiently strong to accelerate CRs. Then, in Appendix C we consider the case of a gas composed of a pre-existing population of CRs and thermal gas and allowing for the acceleration of CRs. The freshly injected CRs obey an equation of state $P_{\text {inj }}=\left(\gamma_{\text {inj }}-1\right) \varepsilon_{\text {inj }}$, where $\gamma_{\text {inj }}=4 / 3$ for an ultra-relativistic CR population that can be accelerated at a strong shock. The injected energy density into CRs, $\varepsilon_{\text {inj }}$, is a constant fraction of the total dissipated energy density at the shock (which is equal to the generated internal energy density corrected for adiabatic compression),

$$
\varepsilon_{\mathrm{inj}}=\zeta \varepsilon_{\mathrm{diss}}=\zeta\left(\varepsilon_{2}-\varepsilon_{1} x_{\mathrm{s}}^{\gamma}\right),
$$

where the compression ratio at the shock is denoted by $x_{\mathrm{s}}=\rho_{2} / \rho_{1}$, the total post-shock energy density is $\varepsilon_{2}=\varepsilon_{\text {inj }}+\varepsilon_{\text {th2 } 2}$, and $\zeta$ is the effective energy injection efficiency after correcting for the fraction of low-energy CRs that is immediately rethermalized by Coulomb interactions with thermal protons. Here, $\varepsilon_{1}$ and $\varepsilon_{2}$ indicate the total energy densities in the upstream and downstream regime of the shock, respectively.

In the following, we summarise the steps which lead to the solution of the Riemann problem, for completeness. Without loss of generality, we assume an initial state with higher pressure in the left half-space. At time $t>0$, the evolving solution is characterised by five regions of gas with different hydrodynamical states which are numbered in ascending order from the right. From the left to right, these regions are separated by the head and the tail of the leftwards propagating rarefaction wave, and the rightwards propagating contact discontinuity and the shock. A Galilean transformation of the Rankine-Hugoniot shock jump conditions from the shock to the laboratory rest system leads to the generalised Rankine-Hugoniot conditions of mass, momentum, and energy conservation at a shock,

$$
\begin{aligned}
v_{\mathrm{s}}[\rho] & =[\rho v], \\
v_{\mathrm{s}}[\rho v] & =\left[\rho v^{2}+P\right], \\
v_{\mathrm{s}}\left[\rho \frac{v^{2}}{2}+\varepsilon\right] & =\left[\left(\rho \frac{v^{2}}{2}+\varepsilon+P\right) v\right] .
\end{aligned}
$$

Here $v_{\mathrm{s}}$ and $v$ denote the shock and the mean gas velocity measured in the laboratory rest system and we defined the abbreviation $[F] \equiv F_{i}-F_{j}$ for the jump of some quantity $F$ across the shock. Note that we assume the pressure of freshly injected CRs to be only nonzero in regime 2 in between the shock and the contact discontinuity. The leftwards propagating rarefaction wave is characterised by an isentropic change of state, $\mathrm{d} s=0$ ( $s$ is the specific entropy), that conserves the Riemann invariant $\Gamma^{+}$:

$$
\Gamma^{+}=v+\int_{0}^{\rho} \frac{c_{\text {sound }}\left(\rho^{\prime}\right)}{\rho^{\prime}} \mathrm{d} \rho^{\prime}=v+\frac{2 c_{\text {sound }}(\rho)}{\gamma-1}=\text { const. }
$$

In the last step, we assumed a polytropic equation of state of the thermal gas, $P=A \rho^{\gamma}$, where $A=$ const. for an isentropic change of state. Appropriately combining these equations, the solution reads as follows:

$$
\begin{gathered}
\rho(x, t)= \begin{cases}\rho_{5}, & x \leqslant-c_{5} t, \\
\rho_{5}\left[-\eta^{2} \frac{x}{c_{5} t}+\left(1-\eta^{2}\right)\right]^{2 /(\gamma-1)}, & -c_{5} t<x \leqslant-v_{\mathrm{t}} t, \\
\rho_{3}, & -v_{\mathrm{t}} t<x \leqslant v_{2} t, \\
\rho_{2}, & v_{2} t<x \leqslant v_{\mathrm{s}} t, \\
\rho_{1}, & x>v_{\mathrm{s}} t,\end{cases} \\
P(x, t)= \begin{cases}P_{5}, & x \leqslant-c_{5} t, \\
P_{5}\left[-\eta^{2} \frac{x}{c_{5} t}+\left(1-\eta^{2}\right)\right]^{2 \gamma /(\gamma-1)}, & -c_{5} t<x \leqslant-v_{\mathrm{t}} t, \\
P_{2}=P_{3}, & -v_{\mathrm{t}} t<x \leqslant v_{\mathrm{s}} t, \\
P_{1}, & x>v_{\mathrm{s}} t,\end{cases} \\
v(x, t)= \begin{cases}0, & x \leqslant-c_{5} t, \\
\left(1-\eta^{2}\right)\left(\frac{x}{t}+c_{5}\right), & -c_{5} t<x \leqslant-v_{\mathrm{t}} t, \\
v_{2}=v_{3}, & -v_{\mathrm{t}} t<x \leqslant v_{\mathrm{s}} t, \\
0, & x>v_{\mathrm{s}} t .\end{cases}
\end{gathered}
$$

Here $\eta^{2}=(\gamma-1) /(\gamma+1), c_{1}=\sqrt{\gamma P_{1} / \rho_{1}}$, and $c_{5}=\sqrt{\gamma P_{5} / \rho_{5}}$ are the speeds of sound in the unperturbed state to the right and left, respectively, $v_{\mathrm{s}}$ is the shock speed and $v_{\mathrm{t}}$ is the speed of propagation of the rarefaction wave's tail in the laboratory system. 
The total post-shock pressure $P_{2}=P_{\mathrm{th} 2}+P_{\text {inj }}$ is obtained by solving (numerically) the non-linear equation for the compression ratio $x_{\mathrm{s}}$, which is derived from the generalised Rankine-Hugoniot conditions over the shock while ensuring the conservation of the Riemann invariant that connects the states 5 and 3 according to equation (B3):

$$
\left[\frac{P_{2}\left(x_{\mathrm{s}}\right)}{P_{1}}-1\right] \frac{\mathcal{A}\left(x_{\mathrm{s}}\right)}{1+\mathcal{A}\left(x_{\mathrm{s}}\right)}-\frac{2 \gamma}{(\gamma-1)^{2}} \frac{c_{5}^{2}}{c_{1}^{2}}\left\{1-\left[\frac{P_{2}\left(x_{\mathrm{s}}\right)}{P_{5}}\right]^{(\gamma-1) /(2 \gamma)}\right\}^{2}=0 .
$$

To derive this equation, we introduce the jump of the thermal pressure across the shock, $y_{\mathrm{s}}$, the ratio of CR-to-thermal energy flux generated at the shock, $\xi=\zeta /(1-\zeta)$, and the Atwood number, $\mathcal{A}$, and find

$$
\begin{aligned}
y_{\mathrm{s}}\left(x_{\mathrm{s}}\right) & =\frac{P_{\mathrm{th} 2}\left(x_{\mathrm{s}}\right)}{P_{1}}=\frac{\xi\left[x_{\mathrm{s}}\left(\gamma_{\mathrm{inj}}-1\right)-\left(\gamma_{\mathrm{inj}}+1\right)\right] x_{\mathrm{s}}^{\gamma}-x_{\mathrm{s}}(\gamma+1)+(\gamma-1)}{\xi\left[x_{\mathrm{s}}\left(\gamma_{\mathrm{inj}}-1\right)-\left(\gamma_{\mathrm{inj}}+1\right)\right]+x_{\mathrm{s}}(\gamma-1)-(\gamma+1)}, \\
\mathcal{A}\left(x_{\mathrm{s}}\right) & \equiv \frac{\rho_{2}-\rho_{1}}{\rho_{2}+\rho_{1}}=\frac{P_{\mathrm{th} 2}\left(\gamma_{\mathrm{inj}}-1\right)+P_{\mathrm{inj}}(\gamma-1)-P_{1}\left(\gamma_{\mathrm{inj}}-1\right)}{P_{\mathrm{th} 2}\left(\gamma_{\mathrm{inj}}-1\right) \gamma+P_{\mathrm{inj}}(\gamma-1) \gamma_{\mathrm{inj}}+P_{1}\left(\gamma_{\mathrm{inj}}-1\right) \gamma}=\frac{y_{\mathrm{s}}\left(x_{\mathrm{s}}\right)+\xi\left[y_{\mathrm{s}}\left(x_{\mathrm{s}}\right)-x_{\mathrm{s}}^{\gamma}\right]-1}{y_{\mathrm{s}}\left(x_{\mathrm{s}}\right) \gamma+\xi\left[y_{\mathrm{s}}\left(x_{\mathrm{s}}\right)-x_{\mathrm{s}}^{\gamma}\right] \gamma_{\mathrm{inj}}+\gamma}, \\
P_{2}\left(x_{\mathrm{s}}\right) & \equiv P_{\mathrm{th} 2}\left(x_{\mathrm{s}}\right)+P_{\mathrm{inj}}\left(x_{\mathrm{s}}\right)=\left\{y_{\mathrm{s}}\left(x_{\mathrm{s}}\right)+\frac{\gamma_{\mathrm{inj}}-1}{\gamma-1} \xi\left[y_{\mathrm{s}}\left(x_{\mathrm{s}}\right)-x_{\mathrm{s}}^{\gamma}\right]\right\} P_{1}, \\
P_{\mathrm{inj}}\left(x_{\mathrm{s}}\right) & \equiv \frac{\gamma_{\mathrm{inj}}-1}{\gamma-1} \xi\left[y_{\mathrm{s}}\left(x_{\mathrm{s}}\right)-x_{\mathrm{s}}^{\gamma}\right] P_{1} .
\end{aligned}
$$

In the limiting case of no $\mathrm{CR}$ injection $(\xi=0)$, it can be shown straightforwardly that equation (B7) reduces to equation (A6) in Pfrommer et al. (2006) for the classical case of a polytropic fluid without CR acceleration. The density on the left of the contact discontinuity is $\rho_{3}=\rho_{5}\left[P_{2}\left(x_{\mathrm{s}}\right) / P_{5}\right]^{1 / \gamma}$, since the gas is adiabatically connected to the left. The post-shock density is simply given by $\rho_{2}=x_{\mathrm{s}} \rho_{1}$. The velocity of the post-shock gas, $v_{2}$, is obtained by combining the rarefaction wave equation, $x / t=v-c$, and the Riemann invariant $\Gamma^{+}$:

$$
v_{2}=v_{3}=\frac{2 c_{5}}{(\gamma-1)}\left[1-\left(\frac{P_{2}\left(x_{\mathrm{s}}\right)}{P_{5}}\right)^{(\gamma-1) /(2 \gamma)}\right] .
$$

Mass conservation across the shock yields $v_{\mathrm{s}}$, and the speed of propagation of the rarefaction wave's tail, $v_{\mathrm{t}}$, is derived with the aid of (B6),

$$
v_{\mathrm{s}}=\frac{v_{2}}{1-\rho_{1} / \rho_{2}} \quad \text { and } \quad v_{\mathrm{t}}=c_{5}-\frac{v_{2}}{1-\eta^{2}}
$$

\section{APPENDIX C: RIEMANN SHOCK-TUBE PROBLEM FOR A COMPOSITE OF COSMIC RAYS AND THERMAL GAS WITH COSMIC RAY ACCELERATION}

\section{C1 Derivation}

A composite gas consisting of CRs and thermal particles does not obey a polytropic equation of state with a constant adiabatic index; only the sup-populations (thermal gas and CRs) fulfil such a relation separately. In the following, we summarise the key considerations that lead to the exact solution of the Riemann shock-tube problem for a composite of thermal gas and CRs (that are adiabatically compressed at the shock) while allowing for CR acceleration at the shock. Accounting for CR shock acceleration yields a composite gas in the post-shock region that is more compressible and experiences an enhanced density jump in comparison to the case without CR acceleration (Pfrommer et al. 2006). To proceed, we adopt the following three approximations: (i) as before, we only consider scales much larger than the CR diffusion length so that we can represent the region surrounding the shock by a discontinuous jump of thermodynamic quantities, (ii) we assume that the pre-existing CR population is adiabatically compressed over the shock and keep the adiabatic index of this CR population, $\gamma_{\mathrm{cr}}$, constant over the shock-tube, and (iii) we assume that the freshly injected CR population obeys the equation of state, $P_{\text {inj }}=\left(\gamma_{\mathrm{inj}}-1\right) \varepsilon_{\mathrm{inj}}$, where $\gamma_{\text {inj }}=4 / 3$ for an ultra-relativistic CR population that can be accelerated at a strong shock. Note that the pressure of freshly injected CRs is only nonzero in the post-shock regime between the shock and the contact discontinuity. Taking $\gamma_{\mathrm{cr}}=$ const. is justified as long as the pre-existing CR pressure is not dominated by trans-relativistic CRs of low energy.

As before, we adopt the convention that the high-pressure state in the initial condition is on the left-hand side. The evolving solution for time $t>0$ is characterised by five regions of gas with different hydrodynamical states which are numbered in ascending order from the right. Starting at the low-pressure state to the right, we encounter the pre-shock region (1), the post-shock region (2), the region trapped in between the contact discontinuity and the rarefaction wave (3), the rarefaction wave itself (4), and the unperturbed high-pressure region to the left (5). We use the notation $P_{2}=P_{\mathrm{inj}}+P_{\mathrm{cr} 2}+P_{\mathrm{th} 2}, \varepsilon_{2}=\varepsilon_{\mathrm{inj}}+\varepsilon_{\mathrm{cr} 2}+\varepsilon_{\mathrm{th} 2}$, $P_{3}=P_{\mathrm{cr} 3}+P_{\mathrm{th} 3}$, and $\varepsilon_{3}=\varepsilon_{\mathrm{cr} 3}+\varepsilon_{\mathrm{th} 3}$ for the total composite pressures and energy densities in the respective regions. The exact solution of the initial value problem requires to determine the time evolution of 14 unknown quantities in the regions (2) and (3): $\rho_{2}, v_{2}, P_{\mathrm{th} 2}, P_{\mathrm{cr} 2}, P_{\mathrm{inj}}, \varepsilon_{\mathrm{th} 2}, \varepsilon_{\mathrm{cr} 2}, \varepsilon_{\mathrm{inj}}$, and $\rho_{3}, v_{3}, P_{\mathrm{th} 3}, P_{\mathrm{cr} 3}, \varepsilon_{\mathrm{th} 3}, \varepsilon_{\mathrm{cr} 3}$ (the behaviour of the rarefaction wave directly follows from these). The regions (2) and (3) are separated by a contact discontinuity, which implies a vanishing mass flux across it and thus, $v_{2}=v_{3}$ and $P_{2}=P_{3}$. The thermal gas obeys a polytropic equation of state, i.e. $\varepsilon_{\mathrm{th} i}=P_{\mathrm{th} i} /\left(\gamma_{\mathrm{th}}-1\right)$ for $i \in\{2,3\}$. This reduces the dimensionality of our problem to 10 unknowns. According to our assumption (iii), the freshly injected CR population obeys a 
polytropic equation of state with adiabatic index $\gamma_{\mathrm{inj}}$ and its energy density, $\varepsilon_{\mathrm{inj}}$, is a constant fraction of the total dissipated energy density at the shock (i.e., the generated internal energy density corrected for adiabatic compression),

$$
\varepsilon_{\mathrm{inj}}=\zeta \varepsilon_{\mathrm{diss}}=\zeta\left(\varepsilon_{\mathrm{inj}}+\varepsilon_{\mathrm{th} 2}-\varepsilon_{\mathrm{th} 1} x_{\mathrm{s}}^{\gamma_{\mathrm{th}}}\right),
$$

where the compression ratio at the shock is denoted by $x_{\mathrm{s}}=\rho_{2} / \rho_{1}$, and $\zeta<1$ is the effective energy injection efficiency after correcting for the fraction of low-energy CRs that is immediately re-thermalized by Coulomb interactions with thermal protons. This reduces the dimensionality by 2 unknowns. Thermal gas and pre-existing CRs are adiabatically expanded over the rarefaction wave and, in our approximation, the pre-existing CRs are adiabatically compressed at the shock, yielding the following relations,

$$
\begin{aligned}
P_{\mathrm{th} 3}=P_{\mathrm{th} 5}\left(\frac{\rho_{3}}{\rho_{5}}\right)^{\gamma_{\mathrm{th}}}, & \\
P_{\mathrm{cr} 3}=P_{\mathrm{cr} 5}\left(\frac{\rho_{3}}{\rho_{5}}\right)^{\gamma_{\mathrm{cr}}}, & \varepsilon_{\mathrm{cr} 3}=\varepsilon_{\mathrm{cr} 5}\left(\frac{\rho_{3}}{\rho_{5}}\right)^{\gamma_{\mathrm{cr}}}, \\
P_{\mathrm{cr} 2}=P_{\mathrm{cr} 1}\left(\frac{\rho_{2}}{\rho_{1}}\right)^{\gamma_{\mathrm{cr}}}, & \varepsilon_{\mathrm{cr} 2}=\varepsilon_{\mathrm{cr} 1}\left(\frac{\rho_{2}}{\rho_{1}}\right)^{\gamma_{\mathrm{cr}}},
\end{aligned}
$$

which further reduces the dimensionality by 5 unknowns. Thus, solving this system requires three more linearly independent equations, two of which are obtained by considering the generalised Rankine-Hugoniot conditions (B2). The last equation is given by the Riemann invariant $\Gamma^{+}$. Using the effective speed of sound, $c_{\text {sound }}=\sqrt{\gamma_{\text {eff }} P / \rho}$, we obtain

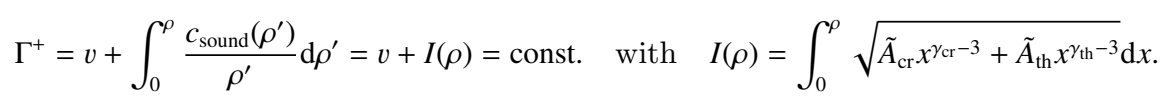

Here, we introduced the abbreviations $\tilde{A}_{i}=\gamma_{i} A_{i}$ where $i \in\{$ th, $\mathrm{CR}\}$ and $A_{i}=P_{i 5} \rho_{5}^{-\gamma_{i}}=P_{i 3} \rho_{3}^{-\gamma_{i}}$ denotes the adiabatic function that is conserved across the rarefaction wave. Defining the difference of the adiabatic indexes of the two populations, $\Delta \gamma \equiv \gamma_{\mathrm{th}}-\gamma_{\mathrm{cr}}$, we obtain the solution to the integral $I(\rho)$,

$$
I(\rho)=\frac{\sqrt{\tilde{A}_{\mathrm{cr}}}}{\Delta \gamma}\left(\frac{\tilde{A}_{\mathrm{cr}}}{\tilde{A}_{\mathrm{th}}}\right)^{\left(\gamma_{\mathrm{cr}}-1\right) /(2 \Delta \gamma)} \mathcal{B}_{x(\rho)}\left(\frac{\gamma_{\mathrm{cr}}-1}{2 \Delta \gamma}, \frac{1-\gamma_{\mathrm{th}}}{2 \Delta \gamma}\right) \quad \text { with } \quad x(\rho)=\frac{\tilde{A}_{\mathrm{th}} \rho^{\gamma_{\mathrm{th}}}}{\tilde{A}_{\mathrm{cr}} \rho^{\gamma_{\mathrm{cr}}}+\tilde{A}_{\mathrm{th}} \rho^{\gamma_{\mathrm{th}}}},
$$

where $\mathcal{B}_{x}(a, b)$ denotes the incomplete beta function. While the second argument of the incomplete beta function is always negative, the expression for $I(\rho)$ is well defined as long as we consider a non-vanishing CR pressure which is characterised by $\tilde{A}_{\text {cr }}>0$ and $\Delta \gamma>0$. For the remaining case $\tilde{A}_{\text {cr }}=0$, the integral can be solved in closed form, yielding $I(\rho)=2 c_{\text {sound }}(\rho) /\left(\gamma_{\text {th }}-1\right)$. Note that the solution of the rarefaction wave fan remains conceptually the same in comparison to the case without CR acceleration (Pfrommer et al. 2006), albeit the wave solution connects to a different $P_{3}$ and $\rho_{3}$, which respond to the softer equation of state in the post-shock regime as a result of $\mathrm{CR}$ acceleration.

\section{C2 Solution of the Riemann problem}

The densities to the left and right of the contact discontinuity, $\rho_{3}$ and $\rho_{2}$, are obtained by matching the possible post-shock states (pressure and density in regime 2) with the possible rarefaction-wave states (regime 3) while simultaneously obeying the conservation laws over the rarefaction wave and the shock. In practice, we have to (numerically) solve the following non-linear system of equations:

$$
\begin{aligned}
& f_{1}\left(x_{\mathrm{s}}, x_{\mathrm{r}}\right) \equiv\left[P_{2}\left(x_{\mathrm{r}}\right)-P_{1}\right]\left(x_{\mathrm{s}}-1\right)-\rho_{1} x_{\mathrm{s}}\left[I\left(\rho_{5}\right)-I\left(x_{\mathrm{r}} \rho_{5}\right)\right]^{2}=0, \\
& f_{2}\left(x_{\mathrm{s}}, x_{\mathrm{r}}\right) \equiv\left[P_{2}\left(x_{\mathrm{r}}\right)+P_{1}\right]\left(x_{\mathrm{s}}-1\right)+2\left[x_{\mathrm{s}} \varepsilon_{1}-\varepsilon_{2}\left(x_{\mathrm{s}}, x_{\mathrm{r}}\right)\right]=0 .
\end{aligned}
$$

Here, we expressed the system of equations in terms of dimensionless density ratios: the shock compression ratio $x_{\mathrm{s}} \equiv \rho_{2} / \rho_{1}$ and the rarefaction wave ratio $x_{\mathrm{r}} \equiv \rho_{3} / \rho_{5}$. The implicit functional dependencies on $x_{\mathrm{s}}$ and $x_{\mathrm{r}}$ read as follows,

$$
\begin{aligned}
P_{2}\left(x_{\mathrm{r}}\right) & =P_{3}\left(x_{\mathrm{r}}\right)=P_{\mathrm{cr} 5} x_{\mathrm{r}}^{\gamma_{\mathrm{cr}}}+P_{\mathrm{th} 5} x_{\mathrm{r}}^{\gamma_{\mathrm{th}}}, \\
\varepsilon_{\mathrm{th}, \mathrm{ad}}\left(x_{\mathrm{s}}\right) & =\varepsilon_{\mathrm{th} 1} x_{\mathrm{s}}^{\gamma_{\mathrm{s}}}, \\
\varepsilon_{2}\left(x_{\mathrm{s}}, x_{\mathrm{r}}\right) & =\left[(1-\zeta) \frac{\gamma_{\mathrm{th}}-1}{\gamma_{\mathrm{inj}}-1}+\zeta\right]^{-1}\left[\frac{P_{2}\left(x_{\mathrm{r}}\right)}{\gamma_{\mathrm{inj}}-1}+\xi \varepsilon_{\mathrm{th}, \mathrm{ad}}\left(x_{\mathrm{s}}\right)-\frac{\gamma_{\mathrm{cr}}-1}{\gamma_{\mathrm{inj}}-1} \varepsilon_{\mathrm{cr} 2}\left(x_{\mathrm{s}}\right)\right]+\varepsilon_{\mathrm{cr} 2}\left(x_{\mathrm{s}}\right)-\xi \varepsilon_{\mathrm{th}, \mathrm{ad}}\left(x_{\mathrm{s}}\right),
\end{aligned}
$$

where $\xi=\zeta /(1-\zeta)$. The post-shock pressure is obtained by inserting the root $x_{r}$ into equation (C6). The post-shock velocity $v_{2}=v_{3}$ and the shock speed $v_{\mathrm{s}}$ are obtained from the generalised Rankine-Hugoniot relations,

$$
\begin{aligned}
& v_{2}=\sqrt{\left[P_{2}\left(x_{\mathrm{r}}\right)-P_{1}\right] \frac{\rho_{2}-\rho_{1}}{\rho_{2} \rho_{1}}}, \\
& v_{\mathrm{s}}=\frac{\rho_{2} v_{2}}{\rho_{2}-\rho_{1}} .
\end{aligned}
$$


Using these results, we find the solution to the generalised Riemann problem for a composite of pre-existing CRs and thermal gas that experiences CR shock acceleration:

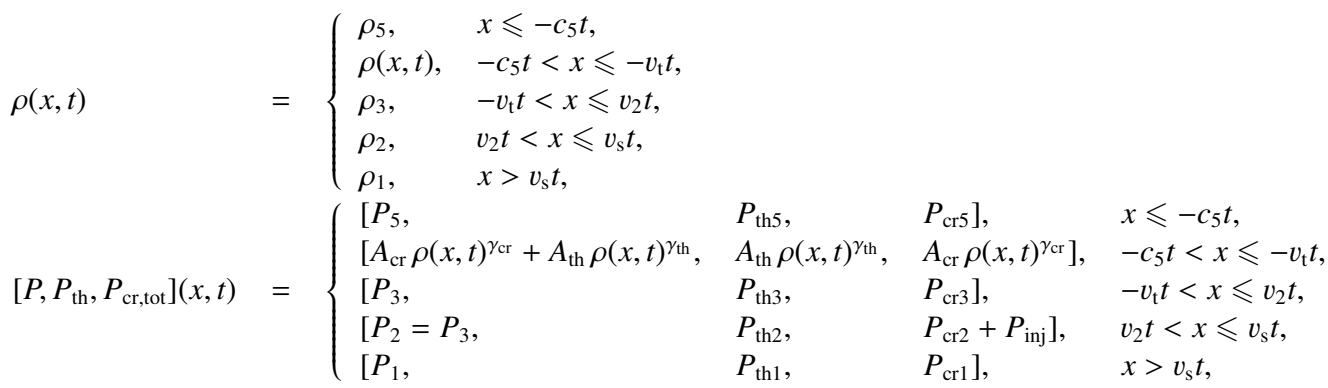

$$
\begin{aligned}
& = \begin{cases}0, & x \leqslant-c_{5} t, \\
\frac{x}{t}+\sqrt{\tilde{A}_{\mathrm{cr}} \rho(x, t)^{\gamma_{\mathrm{cr}}-1}+\tilde{A}_{\mathrm{th}} \rho(x, t)^{\gamma_{\mathrm{th}}-1},}, & -c_{5} t<x \leqslant-v_{\mathrm{t}} t, \\
v_{2}=v_{3}, & -v_{\mathrm{t}} t<x \leqslant v_{\mathrm{s}} t, \\
0, & x>v_{\mathrm{s}} t .\end{cases}
\end{aligned}
$$

Here, $P_{\text {cr,tot }}$ denotes the total CR pressure in a given regime, $c_{5}=\sqrt{\gamma_{\text {eff }} P_{5} / \rho_{5}}$ is the effective speed of sound, $v_{\mathrm{t}}$ is the velocity of the rarefaction wave's tail, and $v_{\mathrm{s}}$ is the shock velocity. Matching the rarefaction wave equation to the density of the post-contact discontinuity (regime 3 ) yields $v_{\mathrm{t}}$ :

$$
v_{\mathrm{t}}=I\left(\rho_{3}\right)-I\left(\rho_{5}\right)+\sqrt{\tilde{A}_{\mathrm{cr}} \rho_{3}^{\gamma_{\mathrm{cr}}-1}+\tilde{A}_{\mathrm{th}} \rho_{3}^{\gamma_{\mathrm{th}}-1}} .
$$

The stratified density within the rarefaction wave (regime 4) is obtained by (numerically) solving the non-linear equation for a given characteristic $(x, t)$, which is derived from the rarefaction wave equation,

$$
I[\rho(x, t)]-I\left(\rho_{5}\right)+\frac{x}{t}+\sqrt{\tilde{A}_{\mathrm{cr}} \rho(x, t)^{\gamma_{\mathrm{cr}}-1}+\tilde{A}_{\mathrm{th}} \rho(x, t)^{\gamma_{\mathrm{th}}-1}}=0 .
$$

Finally, the partial pressures left to the contact discontinuity can be obtain from $(\mathrm{C} 2)$ while the partial pressure quantities in the post-shock region are obtained by the following relations,

$$
\begin{aligned}
P_{\mathrm{th} 2}\left(x_{\mathrm{s}}, x_{\mathrm{r}}\right) & =\frac{1}{1+\zeta}\left[P_{2}\left(x_{\mathrm{r}}\right)+\xi \frac{\gamma_{\mathrm{inj}}-1}{\gamma_{\mathrm{th}}-1} P_{\mathrm{th} 1} x_{\mathrm{s}}^{\gamma_{\mathrm{th}}}-P_{\mathrm{cr} 1} x_{\mathrm{s}}^{\gamma_{\mathrm{cr}}}\right], \\
P_{\mathrm{inj}}\left(x_{\mathrm{s}}\right) & =\xi \frac{\gamma_{\mathrm{inj}}-1}{\gamma_{\mathrm{th}}-1}\left[P_{\mathrm{th} 2}\left(x_{\mathrm{s}}, x_{\mathrm{r}}\right)-P_{\mathrm{th} 1} x_{\mathrm{s}}^{\gamma_{\mathrm{th}}}\right] .
\end{aligned}
$$

In the limiting case of no $\mathrm{CR}$ injection $(\xi=0)$, it can be shown straightforwardly that the system of equations (C5) reduces to equation (B4) in Pfrommer et al. (2006) for the Riemann problem of a composite fluid without CR acceleration.

This paper has been typeset from a $\mathrm{TEX}_{\mathrm{E}} / \mathrm{LAT}_{\mathrm{E}} \mathrm{X}$ file prepared by the author. 